Portland State University

PDXScholar

$1-1-2011$

\title{
Work-related Intimate Partner Violence: The Role of Acculturation Among Employed Latinos in Batterer Intervention Programs
}

Gino Galvez

Portland State University

Follow this and additional works at: https://pdxscholar.library.pdx.edu/open_access_etds Let us know how access to this document benefits you.

Recommended Citation

Galvez, Gino, "Work-related Intimate Partner Violence: The Role of Acculturation Among Employed Latinos in Batterer Intervention Programs" (2011). Dissertations and Theses. Paper 170.

https://doi.org/10.15760/etd.170

This Dissertation is brought to you for free and open access. It has been accepted for inclusion in Dissertations and Theses by an authorized administrator of PDXScholar. Please contact us if we can make this document more accessible: pdxscholar@pdx.edu. 
Work-related Intimate Partner Violence: The Role of Acculturation Among Employed Latinos in Batterer Intervention Programs

by

\section{Gino Galvez}

A dissertation submitted in partial fulfillment of the requirements for the degree of

Doctor of Philosophy

in

Applied Psychology

Dissertation Committee:

Eric Mankowski, Chair

Dalton Miller-Jones

Keith Kaufman

Yves Labissiere

David Ritchie

Portland State University

(C) 2011 


\begin{abstract}
Intimate partner violence (IPV), typically considered in the domestic context, has been shown to have considerable effects on women's employment and health. While the literature has recently grown in this area, very few studies have examined the prevalence of work-related IPV among men. Furthermore, the extant literature on work-related IPV has largely ignored the experience of ethnic minorities, specifically Latinos. Many factors suggest that rates and forms of IPV might be different among other racial and ethnic groups. Some studies that examine IPV among Latinos have sought to understand the role of acculturation and socioeconomic contexts. The purpose of this study was to examine work-related IPV among a sample of men enrolled in batterer intervention programs. In addition, we sought to examine the relationship between acculturation, socioeconomic contexts, and reports of workrelated IPV among a subset of male Latinos.

Overall, the findings confirm the upper ranges of previous estimates across studies (36\% to $75 \%$ ) of employed victims of IPV and their harassment by abusive partners while at work (Swanberg, Logan, \& Macke, 2005; Taylor \& Barusch, 2004). Specifically, we found that $60 \%$ of the entire sample reported work-related IPV that involved threatening behaviors and physical violence at their partner's job. The findings among Latinos suggest that a positive relationship exists between acculturation and work-related IPV. Specifically, proxy variables of acculturation
\end{abstract}


(e.g., country of birth, language of survey, number of years in the U.S.) were hypothesized to be positively associated with higher levels of acculturation. Consistent with the hypotheses, we found significant relationships in the direction proposed.

Lastly, socioeconomic status (e.g., income, education, employment status) was hypothesized to play a moderating role between acculturation and work-related IPV. However, results generally suggest that socioeconomic status (i.e., income, education) did not moderate the relationship between acculturation and work-related IPV. This study makes important contributions to the literature and has implications for employers. The significant rates of work-related IPV found in this study highlight the need to address this problem among employed males as an important step in preventing work-related IPV. Among Latinos, the level of acculturation and factors such as income, employment, and education are important contextual factors that provide a better understanding of IPV in Latino communities (Gryywacz, Rao, Gentry, Marin, \& Arcury, 2009). 


\section{Dedication}

Para mis niñas, Vivian y Violet. 


\section{Acknowledgement}

I would like to sincerely thank my graduate adviser Dr. Eric Mankowski for his support, guidance, intellect, and most importantly, his mentorship during my doctoral work at Portland State University. Dr. Mankowski played a significant role in my development as a research psychologist and I am very grateful for our friendship. There were also other people that contributed to my overall success in graduate school and in particular my dissertation research. I thank my dissertation committee, Dr. Dalton Miller-Jones, Dr. Keith Kaufman Dr. Yves Labissiere, and Dr. David Ritchie for their insight and feedback. My fellow graduate students, Lorraine Escribano, Mika Maruyama, Nan Yragui, Ginger Hansen, Stephanie Morgan, Mary Gray, Ashley Boal, and Margaret Braun deserve recognition for their encouragement throughout this entire process. In addition, I would like to thank my colleagues at the Portland Veterans Affairs Medical Center, Drs. James Henry, Tara Zaugg, Anna Rouse, Garnett McMillan, and Emily Thielman for their support of my dissertation work.

I would like to acknowledge all the support, encouragement, and patience that my family has provided over the years. I have the sincerest gratitude for my parents Julio and Olga and my brothers Julio Jr., Michael, and Matthew. Lastly, I would like to acknowledge my wife Vanessa and our daughters Vivian and Violet for their love and support. My dearest Vanessa, I will always appreciate our partnership and the tremendous amount of patience and understanding that you have shown me over the years. 
Table of Contents

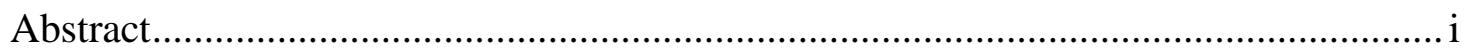

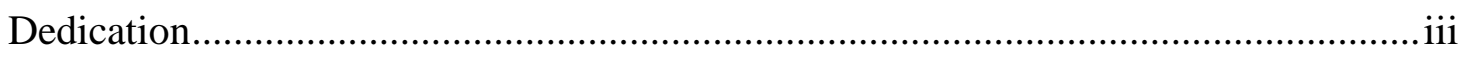

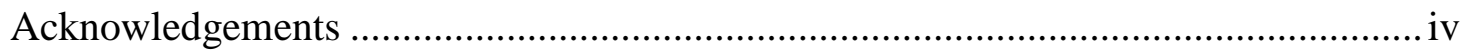

List of Tables ..................................................................................................

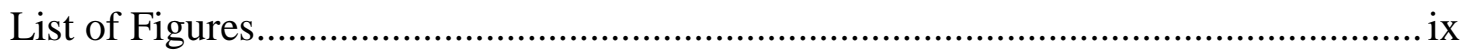

\section{CHAPTERS}

I Introduction and Overview ........................................................................... 1

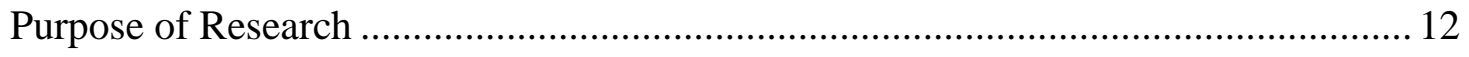

II Review of the Literature ........................................................................... 14

The Cultural Context of Latinos ............................................................................. 14

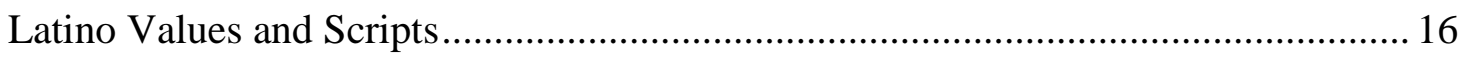

Immigrant Experiences.............................................................................. 21

Intimate Partner Violence Among Latinos ....................................................... 23

Factors Associated with Intimate Partner Violence ............................................ 26

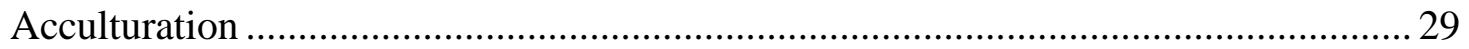

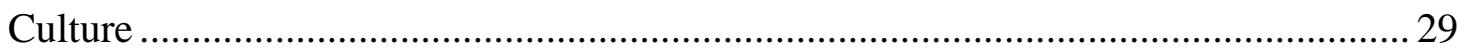

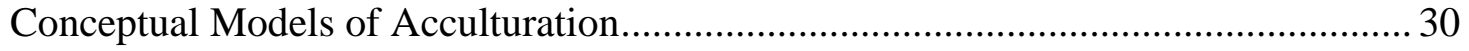

Acculturation and Intimate Partner Violence ....................................................... 33

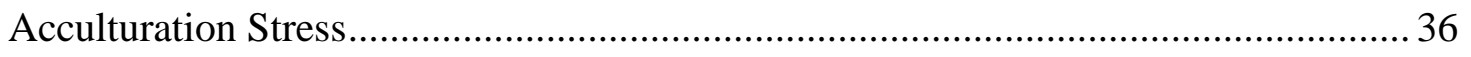




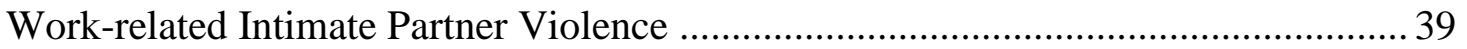

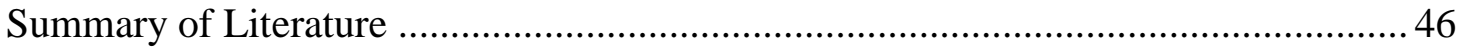

Research Questions and Hypotheses ............................................................. 49

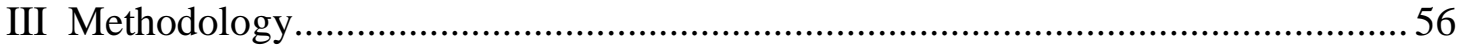

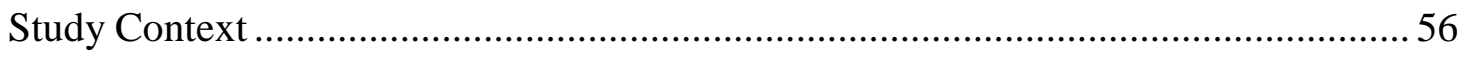

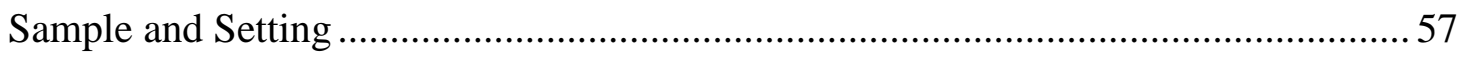

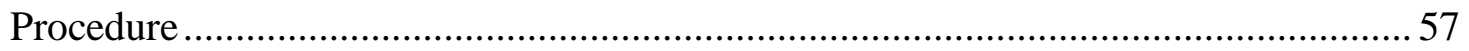

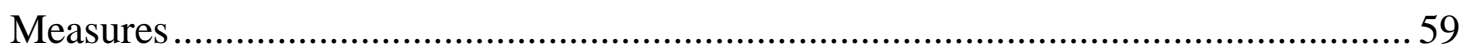

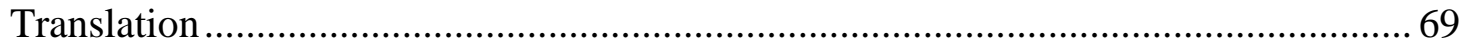

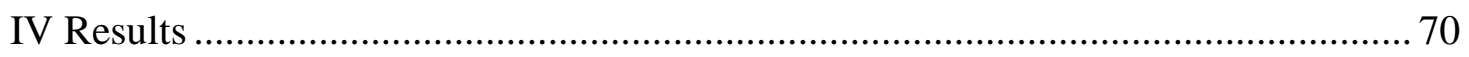

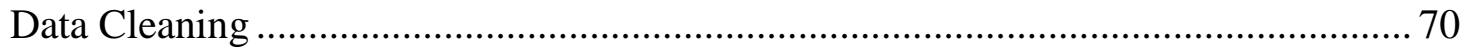

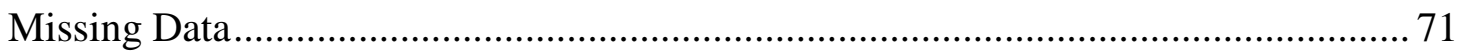

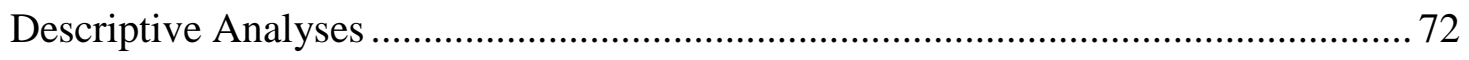

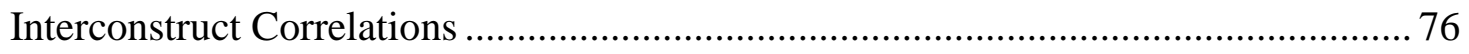

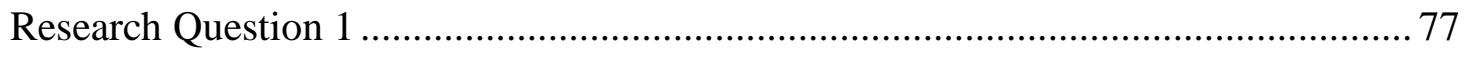

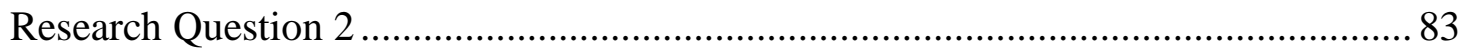

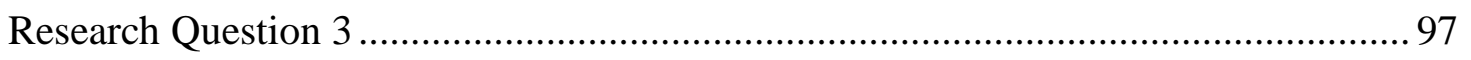

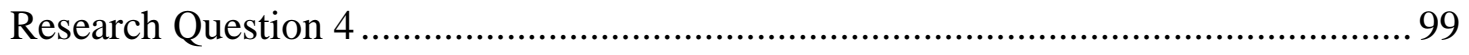

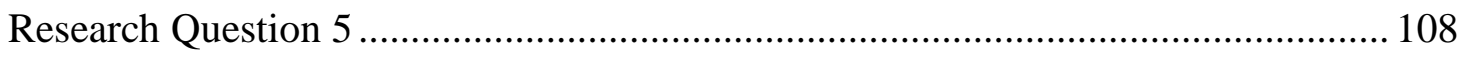

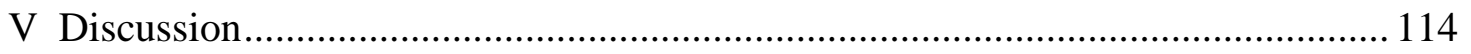

Prevalence of Work-related Intimate Partner Violence........................................ 114 
Ethnicity and Work-related Intimate Partner Violence ........................................ 119

Socioeconomic Status, Ethnicity and Work-related Intimate Partner Violence......... 121

Acculturation and Work-related Intimate Partner Violence................................... 124

Acculturation Status among Couples and Work-related Intimate Partner Violence.. 130

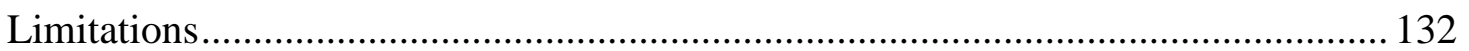

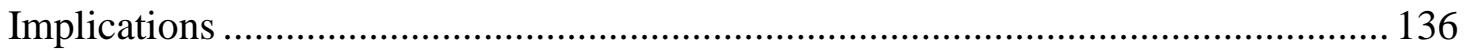

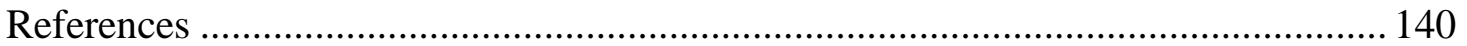




\section{List of Tables}

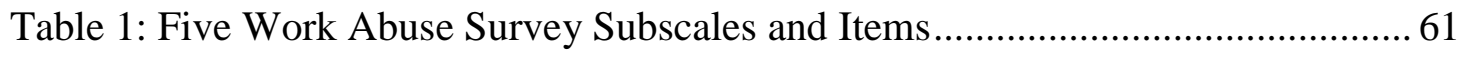

Table 2: Sociodemographic Characteristics of Participants ................................... 74

Table 3: Summary of Means, SDs, and Internal Consistencies for each Construct ..... 75

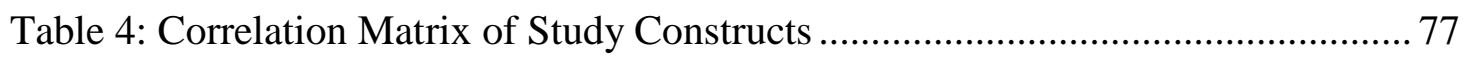

Table 5: Item-level frequency of participants' responses...................................... 79

Table 6: Race Categories of Participants and Work-related IPV ............................. 85

Table 7: Ethnic Subgroups and Affiliations of Participants and Work-related IPV ....86

Table 8: Mexican and White Participants and Reported Work-related IPV ............... 89

Table 9: Multiple Regression for Socioeconomic Indicators on Work-related IPV ....93

Table 10: Multiple Regression for Acculturation Proxy Indicators on Acculturation .99

Table 11: Multiple Regression for Socioeconomic Indicators on Acculturation ....... 101

Table 12: Correlations for Socioeconomic Indicators and Acculturation Indicators . 103

Table 13: Multiple Regression for Acculturation Incongruity on Work-related IPV 109

Table 14: Summary of Results by Research Questions and Hypotheses ................... 111 


\section{List of Figures}

Figure 1: The Moderating Role of Socioeconomic Status Indicators between Ethnicity

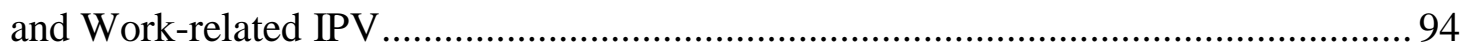
Figure 2: Employment Status Moderates the Relationship between Ethncity and Work-

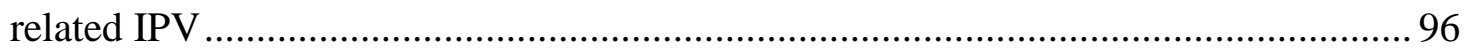

Figure 3: The Moderating Role of Socioeconomic Status Indicators between

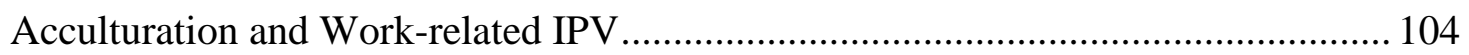

Figure 4: Income Moderates the Relationship between Acculturation (Country of

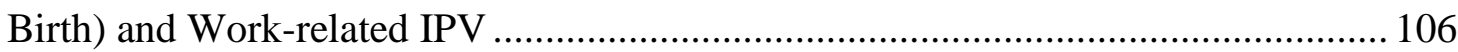




\section{CHAPTER I}

\section{Introduction and Overview}

Intimate partner violence (IPV) is a pervasive social problem in the United States (U.S. Department of Health and Human Services, 2000). IPV results in an estimated 1,200 deaths and 2 million injuries among women each year (MMWR, 2008). Extensive research indicates that IPV poses a significant risk to the physical health of women and it has been associated with increased mortality, injury and disability, poorer health, and other health-related consequences (Plichta, 2004; Tjaden, \& Thoennes, 2000). More recently, IPV has been shown to have considerable effects on women's employment and, over the past decade, there has been a growth in the literature that further examines the relationship between IPV and work (Swanberg, Logan, \& Macke, 2005). Although men are victims of IPV as well, there are important distinctions between men's and women's use of violence. First, research suggests that women are more likely to be victims of IPV than men (Tjaden \& Thoennes, 2000). Also, most studies show that women experience more injuries and burden related to IPV than do men (Archer, 2000; Tjaden \& Thoennes, 2000). Lastly, some studies suggest that female-to-male violence may be retaliatory or defensive in nature (Kimmel, 2002). In light of these differences, this study focuses on male-to-female IPV.

IPV is defined as violence committed by a spouse, ex-spouse, or current or former boyfriend or girlfriend that is carried out with intention, or perceived intention, 
of causing physical pain or injury to another person (National Center for Injury Prevention \& Control, 2003; Straus, Gelles, \& Steinmetz, 1980). The term IPV includes all forms of physical violence, sexual violence, threat of physical or sexual violence, and psychological and emotional abuse (Saltzman, Fanslow, McMahon, \& Shelley, 1999). Some definitions of IPV also include economic abuse. Economic abuse involves behaviors that control a woman's ability to acquire, use, and maintain economic resources (e.g., interfering or preventing the victim from obtaining and maintaining employment), thus threatening her economic security and potential for self-sufficiency (Adams, Sullivan, Bybee, \& Greeson, 2008).

IPV has been shown to have serious workplace implications at the individual (e.g., victimized employees) and organizational levels (e.g., turnover rates) of analysis (Logan, Shannon, Cole, \& Swanberg, 2007; Swanberg, Logan, \& Macke, 2005;

Tolman \& Wang 2005). Some studies have indicated that abusive men use a variety of specific behaviors (i.e., job interference tactics) on intimate partners that interfere with women's ability to get to their work, do their work, and keep their jobs (Riger, Ahrens, \& Blickenstaff, 2000; Rothman \& Perry, 2004; Swanberg, Logan, \& Macke, 2005). Research suggests that work-related IPV affects productivity, work morale, absenteeism, safety and well being of all employees and affects women's relationships with coworkers, supervisors, and customers (Brownwell, 1996; Fitzgerald, Dienenemann, \& Cadorette, 1998; Mighty, 1997; Riger, Ahrens, \& Blickenstaff, 2000). 
The extant literature on work-related IPV has largely ignored the experience of ethnic minorities (Logan, Shannon, Cole, \& Swanberg, 2007; Ridley, Rioux, Lim, Mason, Houghton, Luppi, \& Melody 2005; Rothman \& Perry, 2004; Rothman \& Corso, 2008; Rothman, Hathaway, Stidsen, \& de Vries, 2007; Swanberg \& Macke, 2006; Tolman \& Wang, 2005). In these studies, the samples are predominantly White and in cases in which some ethnic diversity is present, the representation is low, primarily African American, and culture or ethnicity are not addressed at all. Many factors suggest that IPV might be different among other racial and ethnic groups (Klevens, 2007). For example, a longitudinal study found the incidence and recurrence of IPV were higher for Blacks and Latinos than Whites (Caetano, Field, RamisettyMikler, \& McGrath, 2005). Other studies have identified factors associated with ethnic minority status and IPV such as socioeconomic status (e.g., unemployment, poverty) (Cunradi, Caetano, Shafer, 2002; Field \& Caetano, 2004; Kantor, Jasinski, \& Aldorondo, 1994; Neff, Holaman, \& Schluter, 1995). Kantor et al. posit that most researchers erroneously assume that socioeconomic stressors have an equal impact on all groups. Researchers have not yet examined how any of these factors may play a role in work-related IPV among ethnic minorities.

Latinos as an ethnic group merit attention in regards to work-related IPV for many reasons. Latinos constitute approximately $13.3 \%$ of the total population and are the fastest growing minority group in the U.S. and comprise $14 \%$ of the U.S civilian labor force (Day, 1996; Ramirez \& de la Cruz, 2003; U.S. Department of Labor, 
2007). In addition, they are a diverse group that has different immigration histories, family structures, and varying acculturation levels (Bauer, Rodriguez, Quiroga, \& Flores-Ortiz, 2000). Also, Latinos occupy particular employment sectors and experience a host of challenges to gainful employment (e.g., language barriers) all of which may play a role in work-related IPV. As a result of their growth and status, Latinos have become increasingly important to the nation's social structure, culture, and economy (Harris, Firestone, \& Vega, 2005).

As the population of immigrants from Latin American countries increases in the U.S. (Ramirez \& de la Cruz, 2003), it is important to develop a better understanding of Latino health and health-related concerns (Denham et al., 2007). One such concern in the Latino community is IPV. Although IPV is a severe and widespread public health concern that cuts across all races, cultures, and socioeconomic lines, data suggests that it is a prevalent problem in the Latino community (Caetano, Field, Ramisetty-Mikler, \& McGrath, 2005; Kantor, Jasinski, \& Aldorondo, 1994).

In recent years, ethnicity has become particularly relevant in the study of IPV. Researchers have sought to understand the role of ethnicity and IPV because some evidence suggests that rates of IPV are higher for ethnic minorities than Whites. However, other researchers have argued that a better understanding of co-relating factors to ethnicity such as socioeconomic factors (e.g., age, income, and education) is needed in order to appreciate and understand the role that ethnicity may play in the 
occurrence of IPV (Kantor, Jasinski, \& Aldorondo, 1994). Kantor et al. (1994) examined IPV among Latinos and Whites. Their study showed that Latinos did not differ significantly than their White counterparts in the likelihood of IPV against their partners when other factors were held constant (e.g., norms regarding the approval of violence, age, economic stressors). In addition, they demonstrated considerable ethnic variation among Latinos in terms of acculturation, country of birth, impoverishment, and norms regarding the approval of violence. Their study corroborated previous reports suggesting that being born in the U.S. was associated with higher risk of IPV for Latinos than not being born in the U.S. (Sorenson \& Telles, 1991). Country of birth has been shown to be highly correlated with acculturation. Jasinski (1998) suggests that one of the most important factors to understand ethnic variation among Latinos and IPV is acculturation.

Acculturation refers to a lifelong process by which immigrants assimilate and accommodate to the majority culture's way of life (Berry, 1997). It is the continuous contact between two or more distinct groups that causes cultural change in the individual (Berry, 1997). This process includes an evolving relationship to traditional values and beliefs through exposure to the dominant culture (Ho, 1990). This exchange can be beneficial by requiring people to draw on the unique contributions of different cultural backgrounds, but it may also create conflict due to differences in values and interests (Huo, Smith, Tyler, \& Lind, 1996). Acculturation processes 
manifest in a variety of ways such as through changes in socialization, behaviors, and thought processes including language use (Ho).

The level of acculturation may be central to understanding both prevalence rates and forms of IPV among Latinos (Kantor, Jasinski, Aldorondo, 1994). The process of adapting to another culture may result in acculturation stress. Acculturative stress occurs as a result of immigrants experiencing conflict between cultural differences (Hovey \& King, 1996). These cultural differences can cause an individual in the acculturation process to feel torn between their cultural values, norms, and customs and those in the new host culture (Hovey, 2000). Acculturative stress may result in a particular set of emotions and behaviors that may include depression, anxiety, feelings of marginality and alienation, increased psychosomatic responses, and identity confusion (Williams \& Berry, 1991). Among Latinos, some of the stress associated with the acculturation process may create a sense of alienation from their home culture (e.g., Mexican) or magnify the experience of discrimination or deprivation relative to the dominant culture (Kantor, Jasinski, \& Aldorondo, 1994). For example, an exploratory study among Mexican immigrant women showed greater IPV among those with more acculturation stress (Firestone, Lambert, \& Vega, 1999). Acculturation stress may play different roles in both IPV victimization and perpetration among Latinos in the U.S. Firestone, Lambert, and Vega posit that acculturation stress is multidimensional, and specific types of stressors may be more likely to be experienced by immigrants (e.g., fear of apprehension by the Immigration 
and Naturalization Service) than by U.S.-born Latinos (e.g., status inconsistencies, social marginality).

The role of acculturation in the experience and perpetration of IPV has been explored in various studies; however, they have produced mixed findings (Chun \& Akutsu, 2003; Garcia, Hurwitz, \& Kraus, 2005; Jasinski, 1998; Kasturirangan, Krishnan, \& Riger, 2004; Klevens, 2007). A plethora of studies have shown that higher levels of acculturation are associated with higher rates of IPV than less acculturated Latinos (Caetano, Schafer, Clark, Cunradi, \& Raspberry, 2000; Firestone, Lambert, \& Vega, 1999; Ingram, 2007; Kantor, Jasinski, \& Aldorondo, 1994). While other studies have shown an association between higher rates of IPV and lower levels (Sabogal, Marín, Otero-Sabogal, Marín, Perez-Stable, 1987) or lack of acculturation (Champion, 1996), some have shown no differences in rates of IPV when socioeconomic variables were controlled for (Kantor et al., 1994). It has been suggested that the equivocal findings may actually reflect psychometric limitations or different conceptualizations of acculturation (Kasturirangan, Krishnan, \& Riger, 2004; Marín, 1992). However, the findings to date support the notion that an important relationship exists between acculturation and perpetration of IPV, the impact and direction of which still needs to be assessed (Marín \& Gamba, 2003).

Considering the socioeconomic context of Latinos, especially by examining the impact of IPV on their employment, should produce a better understanding of IPV in their community. For example, among Latino immigrants, new economic demands 
may involve shifts in gender roles and power relations that challenge standards of relationships between women and men (Adames \& Campbell, 2005). Research has demonstrated a relationship between employment and IPV (Kantor, Jasinski, \& Aldorondo, 1994). Kantor et al. found that IPV is significantly higher in families of blue-collar workers than for white-collar families. This finding may be particularly relevant for Latinos because they are disproportionately concentrated in blue-collar occupations (U.S. Census Bureau, 2000). In particular, Latinos from a Mexico origin may be more vulnerable to IPV because they have substantial lower mean income levels, higher rates of unemployment, and higher rates of transitional employment than Whites (Becerra, 1988).

Recently, researchers have made progress in addressing the intersection of IPV and employment and have shed light on the associated consequences at the individual (e.g., victimized employees) and organizational levels (e.g., turnover rates) (Logan, Shannon, Cole, \& Swanberg, 2007; Swanberg, Logan, \& Macke, 2005; Tolman \& Wang 2005). Research suggests that abusive men use a variety of specific behaviors (i.e., job interference tactics) on intimate partners that interfere with women's ability to get to their work, do their work, and keep their jobs (Riger, Ahrens, \& Blickenstaff, 2000; Rothman \& Perry, 2004; Swanberg, Logan, \& Macke, 2005). Broadly speaking, research has shown that work-related IPV affects productivity, work morale, absenteeism, safety and well being of all employees and affects women's relationships with coworkers, supervisors, and customers (Brownwell, 1996; Fitzgerald, 
Dienenemann, \& Cadorette, 1998; Mighty, 1997; Riger, Ahrens, \& Blickenstaff, 2000). It has been estimated across studies that between $36 \%$ and $75 \%$ of employed woman who experience IPV are harassed by their abusive partners while at work (Shepard \& Pence, 1988; Swanberg, Logan, \& Macke, 2005; Taylor \& Barusch, 2004).

While a body of research exists that has examined work-related IPV, the intersection of IPV and employment among Latinos and other ethnic minority groups has been largely ignored. In one of the only studies to date that has examined workrelated IPV and Latinos, Galvez, Mankowski, McGlade, Ruiz, and Glass (2011) found that culture informed the participants interpretations of work-related IPV. In this study, focus groups were conducted with employed Latinos enrolled in batterer intervention programs (BIPs) to discuss work-related IPV. The participants discussed their; (a) work-related IPV tactics and behaviors, (b) disapproval of their partners' growing independence (which was perceived as an "American" influence, a violation of traditional gender role expectations for Latinas, and a threat to the preservation of the family), and (c) acculturation stressors in relation to their IPV (e.g., language barriers, lack of economic opportunities). Overall, the findings indicated ways in which acculturation, acculturation stress, and representations of the Latino family may play a role in men's understanding and conceptualization of abusive behavior toward their partners in the workplace. 
Another criticism of the work-related IPV literature is that the majority of studies have relied almost exclusively on victims' reports which ignore information from male abusers (see Rothman \& Perry, 2004 for an exception). While a wealth of information has been procured, the scope of work-related IPV may still be unknown because abusive partners may manipulate, monitor, stalk or harass their partners in ways that go undetected by the victim (e.g., hiding partner's work clothes, tampering with automobile to prevent partner from going to work).

Latino immigrants face a number of challenges that may contribute to IPV or its consequences. For example, lack of information and poor familiarity with service systems, social isolation as a result of having relatives and friends remaining in their country of origin, inaccessibility to services and other means of assistance because of poor English skills, and ineligibility for services because of a lack of appropriate documentation (Cunradi, Caetano, \& Schafer, 2002; Hazen \& Soriano, 2007). These challenges are all likely to make the experience of IPV different for Latinos than other ethnic minority communities because of the distinct sociocultural obstacles that they face (Adames \& Campbell, 2005). Further compounding the socio-structural realities of Latinos in the U.S., researchers have noted the traditional roles, such as males being sole providers and females being stay-at-home wives, have been changing as a result of acculturation (Perilla, Bakeman \& Norris, 1994). These challenges and social inequalities that Latinos experience may produce more stress in their communities, 
which can increase the risk for IPV (Bauer, Rodriguez, Quiroga, \& Flores-Ortiz, 2000).

\section{Summary}

IPV is a pervasive social problem in the United States (U.S. Department of Health and Human Services, 2000). Typically, IPV has been perceived and addressed by researchers as an issue that usually occurs within a domestic context. However, more recently, IPV has been shown to have considerable effects on women's employment, health, and safety (Ridley et al. 2005; Swanberg, Logan, \& Macke, 2005). While the literature has grown in this area, it is incomplete because the experiences of ethnic minority groups have largely been ignored.

Data indicates that IPV is also a prevalent problem among ethnic minorities (Caetano, Field, Ramisetty-Mikler, \& McGrath, 2005; Kantor, Jasinski, \& Aldorondo, 1994). However, researchers have argued that ethnic group differences may be better understood by examining socioeconomic factors (Kantor, Jasinski, \& Aldorondo, 1994). In addition, specifically for Latinos, acculturation may be one of the most important factors to consider in understanding IPV in the Latino community (Jasinski, 1998).

Acculturation refers to a lifelong process by which immigrants assimilate and accommodate to the majority culture's way of life (Berry, 1997). Among Latinos, the level of acculturation may be central to understanding both prevalence rates and forms of IPV (Kantor, Jasinski, \& Aldorondo, 1994). However, studies examining the role of 
Work-related IPV 12

acculturation on IPV have produced mixed findings (Chun \& Akutsu, 2003; Garcia, Hurwitz, \& Kraus, 2005; Jasinski, 1998; Kasturirangan, Krishnan, \& Riger, 2004; Klevens, 2007).

The majority of these studies have shown as association between higher levels of acculturation and higher rates of IPV (Caetano, Schafer, Clark, Cunradi, \& Raspberry, 2000; Firestone, Lambert, \& Vega, 1999; Ingram, 2007; Kantor, Jasinski, \& Aldorondo, 1994). Some researchers have suggested that the equivocal findings may be a result of psychometric limitations (e.g., use of a single proxy to indicate acculturation) or different conceptualizations of acculturation (e.g., unidimensional models) (Kasturirangan, Krishnan, \& Riger, 2004; Marín, 1992).

\section{Purpose of Research}

The purpose of this study is to understand and measure the relationship between acculturation and reports of work-related IPV among employed male Latinos. This study will be one of the first to examine acculturation and other co-related socioeconomic factors as they relate to work-related IPV. Although an increasing number of studies are addressing work-related IPV, the literature in this area has not examined the role of acculturation as a principal factor of interest and its relationship to reported work-related IPV behaviors. Research suggests that Latina victims of IPV underutilize formal services in the legal and healthcare systems because of cultural barriers (e.g., language, low acculturation) (Lipsky, Caetano, Field, \& Larkin, 2006). Addressing IPV in other normative settings (e.g., workplace) that Latinas are already 
participating in may be more effective in addressing IPV (e.g., resources, intervention). The findings of this study could be used to inform the development of culturally-specific IPV interventions focused at the workplace. Furthermore, this study is relevant given the current climate of the need to address the impact of IPV on employment. For example, several states (including Oregon) have recently passed laws regarding employers' responsibilities in addressing IPV (Widiss, 2008).

Following this introductory chapter, Chapter II reviews pertinent literature. Specifically, Chapter II describes the Latino population, culture, and sociocultural contexts (e.g., immigrant experiences). In addition, IPV among Latinos in particular and associated risk factors are discussed. Next, acculturation, acculturative stress, and research examining the link between IPV and acculturation are reviewed. Subsequently, literature examining IPV and employment are reviewed. Chapter III presents the research questions and hypotheses, research design, methodology, and measures used. Chapter IV presents the results of the analyses. Chapter V presents the discussion, limitations, and study implications. 


\section{CHAPTER II}

\section{Review of the Literature}

The traumatizing effects of IPV cut across all races, cultures, and socioeconomic lines. However, many factors suggest that rates and forms of IPV might be different among other racial and ethnic groups (Klevens, 2007). Latinos are such a group that faces numerous barriers and sociocultural contexts that uniquely influence and shape their experience of IPV. In particular, this review will provide the context for IPV and Latinos by describing the Latino population in the U.S., and various culturally-specific values (e.g., familismo), scripts (e.g., simpatia), and social contexts (e.g., immigrant experiences). In addition, the prevalence of IPV among Latinos and associated risk factors are discussed. Subsequently, acculturation and acculturation stress research that examines the relationship to IPV are discussed. Finally, literature examining IPV and employment, including abuse tactics and strategies that abusers use are reviewed.

\section{The Cultural Context of Latinos}

Over 37.4 million people in the United States identified themselves as Latino (i.e., Hispanic, Spanish, or Latino; of all races) in the 2002 U.S. Census Current Population Survey (Ramirez \& de la Cruz, 2003). Latinos constituted approximately $13.3 \%$ of the total population and are the largest and fastest growing ethnic minority group in the U.S. (Day, 1996; Ramirez \& de la Cruz, 2003; U.S. Census Bureau, 2000). Projections made by the Pew Hispanic Center suggest that the Latino 
population will triple in size (29\%) by 2050 and will account for most of the nation's population growth from 2005 through 2050 (Passel \& Cohn, 2008). The majority of Latinos in the U.S. are of Mexican origin (67\%), Central and South American (14.3\%), Puerto Rican (8.6\%), Cuban (3.7\%), and other (6.5\%; Ramirez \& de la Cruz, 2003). More than $40 \%$ of the Latinos in the U.S. were born in other countries (Ramirez \& de la Cruz, 2003), which suggests that a significant proportion may be experiencing culture change (i.e., acculturation).

The terms "Latino" and "Hispanic" have typically been used in the U.S. to describe groups of people whose heritages originate from Spanish speaking countries of Latin America. The term "Hispanic", however has been used without much regard to the heterogeneity that exists among the cultural groups from Latin America and has therefore contributed to some misperceptions and misunderstandings. For example, some ethnic groups from Central and South America do not identify with and are offended by the term "Hispanic" because the label has been associated with Spain, which played an oppressive role in their history (Malley-Morrison \& Hines, 2004).

Another concern related to the use of the term "Hispanic" is that many researchers have mistakenly used it as a racial label. This is an important concern because it is very possible that an individual may be ethnically "Hispanic" and racially Black or White at the same time (Marín \& Marín, 1991). Conversely, the term "Latino" describes diverse cultural groups that are not necessarily linked to any race or religion and it is preferred more so than the term "Hispanic" because it is more 
inclusive (Fontes, 2002; Malley-Morrison \& Hines, 2004). As a result, the term "Latino" will predominantly be used throughout this study and the study design includes questions both about ethnic groups (e.g., Mexican, Puerto Rican) and race (e.g., Black, Asian).

\section{Latino Values and Scripts}

While there is much diversity among Latinos, it is widely believed that members of this group share some common basic cultural values and social scripts (Mirandé, 1997). Marín \& Marín (1991) examined a variety of sources and proposed some commonalities among Latinos. For example, familismo, simpatia, machismo, and marianismo were proposed as important values and social scripts that are shared by most Latinos regardless of their national background, birthplace, dominant language, or any other sociodemographic characteristic (Marín \& Marín, 1991). A brief discussion of these aforementioned cultural values and social scripts is needed for two reasons. First, they provide important context for Latinos and implications to IPV (Malley-Morrison \&Hines, 2004). Second, many of these values (e.g., familismo) have been linked to the acculturation process and their inclusion may shed light on the relationship between acculturation and IPV.

Familismo (or familism) is a cultural script that refers to the strong identification and attachment to nuclear and extended family members (Sabogal, Marín, \& Otero-Sabogal, 1987). More specifically, familismo involves (1) perceived obligation to provide material and emotional support to the members of the extended 
family; (2) reliance on family members for help and support; and (3) the perception of family members as behavioral and attitudinal referents (Marín \& Marín, 1991). Familismo is a distinctive characteristic for Latinos and some aspects (e.g., sense of belonging, reciprocity, and obligation) remain fairly strong across generations who experience acculturation. However, there is some research that suggests that behaviors and attitudes related to familismo weaken with increasing contact with U.S. mainstream culture (Sabogal, Marín, \& Otero-Sabogal, 1987). In a sense, familismo is a structure for Latino familial relationships. Simpatia is a related concept which is defined as a preference for positive interpersonal interactions (Triandis, Marín, Lisansky, \& Betancourt, 1984) and it facilitates smoother and more positive interactions within these relationships. More specifically, simpatia is associated with conformity and empathy for the feelings of other people. In addition, it requires individuals to behave with dignity and respect towards others and to strive to achieve harmony in personal relations and avoid interpersonal conflict (Griffith, Joe, Chatham, \& Simpson, 1998). Research has shown that Latinos in particular tend to avoid confrontation and interpersonal conflict (Triandis et al., 1984).

Machismo is a construct of masculinity for Latino males that is commonly represented as various forms of domineering behavior, promiscuity, excessive use of alcohol, aggression, bravery, sexism, oppressive and controlling behaviors, emotional restriction, and maltreatment of children (Marín \& Marín, 1991; Mayo, 1997; Valdés, Barón, \& Ponce, 1987). However, machismo has been largely misrepresented by 
scholars, writers, researchers, and media by over emphasizing negative aspects. An expanded view of machismo incorporates many positive aspects that Latinos themselves identify more with than the negative aspects (Torres, Solberg, Scott, Carlstrom, \& Aaron, 2002). From this perspective, machismo is something to be proud of because it frames men as the head of the family, nurturing, supportive, familyoriented, hard working, courageousness, charitable, and as a protective role of the family (Falicov, 1998; Mayo, 1997; Torres, 1998; Torres et al. 2002; Valdés, Barón, \& Ponce, 1987). Some research suggests that machismo is weaker among more acculturated Latinos (Mayo, 1997).

These aspects of machismo are important to understand because they might be interpreted by Latinos in different ways that either increase or decrease the potential of IPV among Latino couples. For example, men who adopt the positive perspective of machismo may not physically abuse their partners because they perceive their role as their protector and abusing their partner would be incongruent with their role and would make them feel ashamed. In contrast, from the stereotypical perspective of machismo, Latinos may believe that their partners are not equal and must be loyal and obedient to them and use physical abuse as a way to exert dominance and control.

Marianismo is a gendered script which is derived from the Virgin Mary that represents the female archetype for all Latinas. In Roman Catholicism, the Virgin Mary is revered and worshipped because she is virtuous, immaculate, and most importantly, is the quintessential mother. Marianismo requires Latinas to emulate the 
Work-related IPV 19

Virgin Mary's moral integrity and spiritual strength, which essentially manifests as a self-sacrificing role for their families (Stevens, 1973). This belief enables women to believe that they are spiritually superior to men and thereby capable of enduring suffering caused by their husbands or partners (Stevens, 1973; Comas-Diaz, 1995). Latinas are expected to possess virgin-like qualities, be humble, and should tolerate their husbands' bad habits, comply with their husbands' demands, and support their husbands in an unwavering manner (Campbell \& Lewandowski, 1997). Cultural pressures, like marianismo may make it difficult for women to leave abusive relationships (Kasturirangan, Krishnan, \& Riger, 2004). For example, Latinas who had experienced IPV reported a strong sense of cultural pressure to remain in an abusive relationship and were afraid of what other people would say about leaving their marriage (Kasturirangan \& Williams, 2003). Malley-Morrison and Hines (2004) argue that Latinas in some ways accept and tolerate IPV because they have been socialized to accept the perception that male partners have a right to physically discipline and make demands of them while requiring an unquestioning loyalty. The cultural pressure to remain in relationship may be unique to Latinas and it may engender more IPV. Furthermore, it's possible that Latinas may stay in abusive relationships longer because of marianismo than other groups of women which could help explain some of the data that suggest higher IPV among Latinos than Whites (Caetano, Field, Ramisetty-Mikler, \& McGrath, 2005). 
Work-related IPV 20

Familismo, simpatia, machismo and marianismo are important cultural values and social scripts that provide context for Latinos in the U.S. While some evidence suggests that these cultural values and social scripts are universal to all Latinos, there is much heterogeneity among Latinos in the U.S. For example, a national survey of 2,544 Latinos in the U.S. found a wide-range of diversity among the different Latino subgroups in terms of immigration, differing histories of relationships between Latinos' home countries and the U.S., language use, changing family relationships, and changing social contexts (Guamaccia, Pincay, Alegria, Shrout, Lewis-Fernandez, \& Canino, 2007). Latino subgroups may differ in many ways and these differences may influence and shape cultural values and social scripts. Furthermore, culture evolves and changes and broad characteristics of ethnic groups are always problematic because it assumes that cultures are static.

Another problem with describing broad characteristics of Latinos is the possibility of interpreting these characteristics as causes of IPV in Latino communities. The cultural values and social scripts reviewed in this study provide cultural context, however, they should not be interpreted as causing IPV among Latinos. In fact, some researchers are careful to avoid suggesting causal links between culture and IPV because doing so may create and sustain negative inaccurate stereotypes (Kantor \& Jasinski, 1998). Also, cultural explanations of IPV ignore larger socio-structural contexts (e.g., poverty) that have been linked to IPV (Kantor, Jasinski, \& Aldorondo, 1994). 
Work-related IPV 21

\section{Immigrant Experiences}

One commonality that many Latinos living in the U.S. (and in Oregon) experience is immigration. Approximately $40 \%$ of Latinos in the U.S. were born in other countries and more than half immigrated between 1990 and 2002 (Ramirez \& De La Cruz, 2003). The process of immigration is typically very difficult and traumatic because Latinos face a multitude of social and economic barriers that hinder their ability to adapt and cope within their new environment. Latinas experience a host of stressors related to the immigration process that include loss of family and friends (social isolation), difficulty in language acquisition, limited information and education, limited access to resources, unfamiliarity with the U.S. culture and legal system, and immigration status (i.e., undocumented) all of which can cause difficulties in seeking help and leaving abusive partners (Orloff \& Little, 1999).

In particular, the loss of support networks (i.e., extended family) may have detrimental impact for immigrants experiencing IPV. For instance, immigrating to the U.S. typically involves leaving behind support networks (i.e., extended families) in their country of origin (e.g., Mexico). Given that Latinos have a strong identification and attachment to nuclear and extended family members (Sabogal, Marín, \& OteroSabogal, 1987), the loss of support networks may increase the risk of IPV among Latinas. Vasquez (1998) noted that the support that the extended family offers can protect individual members against IPV. For many Latina immigrants, the loss of support networks can weaken their ties to their home culture and create a sense of 
Work-related IPV 22

isolation in their new environment. For example, immigrant women who feel isolated from the mainstream culture may increase their reliance on their partner to navigate everyday situations in a new country (Bui \& Morash, 1999; Erez \& Hartley, 2003). Isolation coupled with a loss of social networks may make a woman more vulnerable to IPV because others are not there to discourage IPV as it develops, provide emotional support, and resources (e.g., financial support, safe environment) to leave an abusive partner.

For Latinas who experience IPV, leaving an abusive partner may be more difficult because the male partner may be the only source of financial support and may be their only Spanish-speaking relationship in the U.S. which further increases Latinas social isolation and dependence. For instance, an Oregon-based report (Oregon Human Development Corporation, 2003) identified several barriers to leaving an abusive relationship specifically among Latinas who had experienced IPV. The most commonly reported barriers were (1) economic dependence on the abusive partner; (2) lack of legal status; and (3) language, cultural and family pressure to stay in the abusive relationship.

Undocumented immigrant women residing in the U.S. may be at further risk of IPV than those who have U.S. citizenship. Current estimates suggest that there are 9.3 million undocumented immigrants residing in the U.S. Latinos of Mexico-origin in particular make up over half $(57 \%)$ or about 5.3 million people, another $23 \%$ are from other Latin American countries or 2.2 million and lastly about $25 \%$ are from other 
countries (Passel, Capps, \& Fix, 2004). Undocumented immigrants typically refer to two types of groups: (a) those who entered the country without valid documents; and (b) those who entered with valid visas but overstayed their visas' expiration or otherwise violated the terms of their admission (Passel, Capps, \& Fix).

Some undocumented immigrant women face threats of deportation from their abusive partner (Campbell, Masaki, \& Torres, 1997; Erez \& Hartley, 2003). Abusive partners of immigrant women may use threats related to deportation in an effort to maintain power and control over their partner (Shetty \& Kaguyutan, 2002). For example, abusive partners may threaten to report them to the Immigration and Naturalization Service (INS), rescind residency sponsorship, or refuse to file important INS documents (Shetty \& Kaguyutan, 2002). However, threats related to deportation are an omnipresent weapon for abusers to use against their immigrant partners, regardless of their partners' immigration status (Erez \& Hartley, 2003).

\section{Intimate Partner Violence among Latinos}

The lifetime prevalence of IPV among Latinas has been estimated in the range of $10 \%$ to $20 \%$ (Denham et al. 2007; Lown \& Vega, 2001; Tjaden, \& Thoennes, 2000). While this area of inquiry is still growing, some research has shown that a disproportionate number of ethnic minorities, specifically Latinos, experience higher rates of IPV than non-Latinos (Caetano, Field, Ramisetty-Mikler, \& McGrath, 2005; Ingram, 2007; Kantor, Jasinski, \& Aldorondo, 1994; Sorenson \& Telles, 1991). Among a national sample of ethnically homogenous couples (i.e., Whites, African 
Work-related IPV 24

Americans, and Latinos), Caetano and colleagues (2005) found that the prevalence, incidence, and recurrence of IPV were significantly higher for Latinos (25\%, 14\%, $58 \%$, respectively) than Whites $(11 \%, 6 \%, 37 \%$, respectively) in 2000 . Furthermore, compared to Whites, Latinos were 2.5 times more likely to initiate IPV between baseline and a five year follow-up. In addition, they found the rate of recurrence for severe IPV was four times higher among Latinos than the rate among Whites. While some national probability samples and local population-based surveys have found higher rates of IPV among Latinos, several other reports have either contradicted previous estimates (Bauer, Rodriguez, \& Perez-Stable, 2000; Sorenson, Upchurch, \& Shen, 1996) or shown similar rates (Denham et al., 2007; Kantor, 1997; Tjaden, \& Thoennes, 2000). For example, among a diverse sample of 1212 women in blue-collar work sites, Denham et al. (2007) found an adult lifetime prevalence rate of IPV of 20\% for Latinas, 25\% for Whites, and 32\% for African Americans. While estimates for this study regarding Latinas is comparable to another report based on the National Violence Against Women Survey (Tjaden, \& Thoennes, 2000), overall prevalence rates have varied. Hazen \& Soriano (2007) recently found high lifetime rates of IPV among 292 Latinas (43.1\% U.S. born, 40.1\% immigrant, and $16.8 \%$ migrant-seasonal workers). Specifically, they found that $33.9 \%$ reported physical violence, $20.9 \%$ reported sexual coercion, and $82.5 \%$ reported psychological aggression. 
Work-related IPV 25

Denham et al. (2007) suggest that the variation in estimates across studies is influenced by the specific population (e.g., Latinos as a group, Latino subgroups, rural or urban samples) being studied or the time interval for which victims are being asked to report IPV (e.g., past 6 months, lifetime). For example, a study by Ingram (2007) demonstrated different rates depending on the time interval of IPV (e.g., lifetime prevalence, past 12 months). Among 1,973 Latinos (47.4\% male; 52.6\% Female) and 9,982 non-Latino Whites (47.7\% Male; 52.3\% Female), Ingram found significantly fewer Latinos had experienced a lifetime prevalence of IPV (50.6\%) than non-Latinos (58.5\%). However, a significant difference in higher rates of IPV was found among Latinos (18.7\%) than non-Latinos (15.7\%) who reported experiencing IPV in the past 12 months.

It is important to note that there is a history of comparative research that has caused great harm to many ethnic groups in the U.S. For example, historical research on race, intelligence, and health was largely racist, unethical, and ineffective (Bhopal, 1997). In many cases, this type of research has perpetuated false stereotypes of ethnic groups (e.g., the perception that the health of ethnic minority groups is poor). However, comparative research or reports of IPV prevalence among different ethnic groups are useful for many reasons and if conducted properly may help groups that are disproportionately affected by IPV. Research that takes in to account for example socioeconomic indicators, the comparison of similar groups (e.g., rural vs. rural), acknowledges cultural context, clearly defines ethnic samples, and describes 
limitations of the data specific to ethnic groups are more likely to produce valuable knowledge and less likely to perpetuate stereotypes. For example, Straus \& Smith (1995) clearly outlined important limitations of their study which found higher rates of IPV among Latinos than Whites (e.g., did not measure country of origin, data collected only by phone and in the English language). In addition, they strongly drew attention to the possibility that the findings could be misused and misrepresented by bigoted and prejudiced persons. Research that examines Latinos should be approached with an understanding of the intersectionality of the social forces (e.g., poverty) that are at work in the occurrence of IPV in Latino populations and communities (Carrillo \& Zarza, 2008).

\section{Factors Associated with IPV}

Of the studies that exist that examine IPV among Latinos, some attention has been paid to understand factors associated with the occurrence of IPV. West, Kantor, and Jasinski (1998) examined sociodemographic factors among a sample of 193 women (76 Latina, 117 White) who experienced IPV. Latinas in their study were significantly younger, less educated, and more economically disadvantaged (i.e., below poverty level) than their White counterparts. While both Latinas and White women were equally likely to experience severe forms of IPV, the combination of lower income and lower educational attainment, make Latinas more isolated, vulnerable, and at a higher risk for IPV. 
Work-related IPV 27

Economic factors have consistently been shown to be related to Latinos' experience of IPV. In particular, low income has been associated with higher rates of IPV (Cunradi, Caetano, \& Schafer, 2002; Pearlman, Zierler, Gjelsvik, VerhoekOftedahl, 2003; Straus \& Smith, 1995; Sugihara \& Warner, 2002; Torres, 1991). Among 1440 couples (555 White, 527 Latino, 358 African American), Cunradi et al. found significant lower mean annual household income among Latino couples who reported male-to-female partner violence than those who did not. However, Latino couples who reported female-to-male partner violence did not significantly differ in mean annual incomes than couples who did not report female-to-male partner violence. Generally, these findings support previous research that link low socioeconomic status with increased risk of IPV.

Research on normative samples (i.e., White middle class) has established an association between education and IPV (Gelles, 1985) and it appears that that relationship holds for Latinos as well. For example, Kantor, Jasinski, and Aldorando (1994) found that Latino families who reported IPV are significantly more educationally disadvantaged than non-Latino families. However, Cunradi, Caetano, and Schafer (2002) found that income had more of an influence on the probability of IPV than education. That being said, both income and education are associated with the ability to avoid detection of criminal activity (i.e., IPV) or prosecution by the criminal justice system. For Latinas, the relationship between educational attainment 
Work-related IPV 28

and IPV is not yet clear and more research is needed in order to understand its impact (Denham et al., 2007).

Regarding Latino couples, Latinas who have experienced IPV are more likely than their White counterparts to categorize their marriages as being male-dominated (West, Kantor, \& Jasinski, 1998). Sugihara and Warner found that among 316 Mexican Americans (161 males, 155 females), decision-making power (e.g., authority) was associated with physical assault and high devaluation (e.g., disparagement) with inflicting injury among men. In other words, men tended to have more decision-making power and tended to look down upon female partners more than women. However, their research suggests that dominance among Latinos is not exclusive to Latino males. Both men and women in their study reported high levels of dominance which were associated with IPV. Their nuanced findings are consistent with previous literature that suggests that Mexican American men and women are realizing more egalitarian gender roles, although some traditional gendered behaviors are retained in their relationships (Mirandé, 1997).

Although some aspects of Latino gender roles have become more egalitarian (e.g., household division of labor), other traditional aspects have remained, especially regarding the role of males as primary income providers. Among Latinas, some research suggests that Latinas who earn or contribute more income than their partners appear to be at greater risk of IPV (Morash, Bui, \& Santiago, 2000; Perilla, Bakeman, \& Norris, 1994). IPV might occur as a result of changes within traditional gender roles 
or when they become destabilized (Klevens et al., 2007; Morash, Bui, \& Santiago, 2000). For example, Morash et al. (2000) found that physical and emotional forms of abuse were more likely to occur in families where gender roles had changed and when expectations were incongruent with performance. However, the link between traditional gender role attitudes and IPV is generally weak (Sugarman \& Frankel, 1996). More research is needed to clarify the importance of gender role strain as a result of immigration or acculturation as an associated factor of IPV among Latinos (Klevens, 2007).

\section{Acculturation}

Immigration is a common experience for many Latinos in the U.S. and it often implies acculturation. Acculturation refers to a lifelong process by which immigrants assimilate and accommodate to the majority culture's way of life (Berry, 1997). It is the continuous contact between two or more distinct groups that causes cultural change in the individual (Berry, 1997). This exchange can be beneficial by requiring people to draw on the unique contributions of different cultural backgrounds, but it may also create conflict due to differences in values and interests (Huo, Smith, Tyler, \& Lind, 1996).

\section{Culture}

The concept of culture is central to the conceptualization of acculturation (Hunt, Schneider, \& Comer, 2004). A brief discussion of the concept of culture needs to be included in order to provide sufficient context to understand the conceptual 
process of acculturation. Culture has been described as the human-made part of the human environment and the total heredity of mankind which includes knowledge, belief, art, laws, customs, and capabilities and habits acquired by humans as members of society (Herskovitz, 1948; Kroeber \& Kluckholn, 1952; Linton, 1936; Tylor, 1871). While various definitions of culture have been developed and refined over the years, there is a general understanding that culture is comprised of both concrete, observable activities and artifacts, and of underlying symbols, values and meanings (Berry, Poortinga, Segall, \& Dasen, 2002). Generally, culture has been constructed from factors such as language, activities, skills, behaviors, level of acculturation and so on. In research, the factors that should be studied in order to understand culture depend on how culture is defined because different definitions imply different approaches, methods, and research questions (Toomela, 2003).

\section{Conceptual Models of Acculturation}

There are two theoretical perspectives that have dominated the explanation of the complex process of acculturation in the literature; (1) unidimensional or (2) bidimensional models of acculturation (Cabassa, 2003). A unidimensional model of acculturation places an individual's movement along a single continuum, ranging from the immersion in an individual's own culture of origin to the immersion in the dominant or host culture (Cabassa, 2003). From this perspective, the acquisition or adherence to a new dominant culture and the maintenance of the original culture are considered part of the same phenomenon. 
Work-related IPV 31

Alternatively, the acculturation process can also be considered as a process in which individuals or groups change on more than one dimension (Berry, 2003). That is, acculturation can also be conceptualized as a multidimensional and multidirectional process whereby, for example, Latinos absorb, learn, acquire, and integrate the overt and covert cultural characteristics of the dominant host culture (Valdés, Barón, \& Ponce, 1987). In many cases, successful adaptation among acculturating immigrants depends on how and whether they retain their own ethnic identity and/or value interactions and absorb the cultural norms of the dominant culture (Berry, Trimble, \& Olmedo, 1986; Berry, 1997). In addition, the acquisition or adherence to a new dominant culture is independent of the maintenance of the original culture (Cabassa, 2003). The amount of participation and contact the individual has with the new dominant culture can also vary as well. Therefore, both dimensions (i.e., maintenance of one's own culture, participation and contact with other groups in larger society) can range from full participation to full rejection of either culture's values, behaviors, and attitudes (Berry, 2003; Cabassa, 2003).

According to Berry (1997), varying along the two dimensions of (a) preference for maintenance of one's own culture and (b) preference for participation and contact with other groups in larger society, will yield four acculturation strategies or states: (a) integration, to retain their own ethnic identity and value interacting with members of the host society; (b) assimilation, the abandonment of their ethnic identity and adopting the cultural norms of the host society (as well as value interacting with it's 
Work-related IPV 32

members); (c) separation, to retain their own ethnic identity and not value interacting with members of the host society; (d) marginalization, to not retain their own ethnic identity as well as not value interacting with the host society. While this model is useful and included in this review in order to further the understanding of acculturation as a multidimensional process, most research has not yet examined the four acculturation strategies in regards to IPV among Latinos. Most research has considered acculturation as a unidimensional process that places an individual's movement along a single continuum.

Marín (1992) proposed that "culture learning" is part of the acculturation process. Specifically, "culture learning" refers to three distinct and gradual levels of change an individual undergoes in the acculturation process. The first level involves the learning (and forgetting) of the facts that are part of one's cultural history or tradition which can include changes in the consumption of food and use of media (e.g., television programs). The second level involves culturally-specific behaviors that are central to a person's social life, which can include such things as language preference and use (e.g., Spanish), and preference for the ethnicity of members in social networks, and media (e.g., Spanish language television programs). Finally, the last level includes the adoption of values and norms of the dominant culture, which can also include the maintenance of the original culture's norms.

For Latinos, adapting to a new environment may have unique implications for IPV. The process of adapting is typically very difficult for Latinos because they are 
faced with many challenges, such as adjusting to a new language, different customs and norms for social interactions, lifestyle changes (e.g., rural to urban), and unfamiliar rules and laws (Balls Organista, Organista, \& Kurasaki, 2003). For example, the relativity low income of Latino men in the U.S. has been theorized to play a role in IPV. That is, researchers have speculated that underemployment and poverty lead to IPV because the man is not able to fulfill his role as a sole provider and must "stoop" to letting his partner help with the earning an income (Zambrano, 1985). Generally, researchers have sought to understand various changes (in values, cultural scripts, gender roles) that Latinos experience as a result of residing in the U.S. vis-à-vis the acculturation process. The role of acculturation on IPV has been explored in various studies (e.g., associations to health outcomes). However, the research on the relationship between acculturation and IPV has produced equivocal findings (Chun \& Akutsu, 2003; Garcia, Hurwitz, \& Kraus, 2005; Jasinski, 1998; Kasturirangan, Krishnan, \& Riger, 2004; Klevens, 2007). Some studies suggest that acculturation does not protect against IPV and in some instances may even increase risk for of abuse.

\section{Acculturation and Intimate Partner Violence}

Kantor, Jasinski, and Aldorondo (1994) sought to examine the relationship between both cultural and socioeconomic status indicators and wife assault among 743 Latinos. Acculturation was not significant when normative approval of wife-slapping, country of birth, and poverty were considered. However, place of birth (which is 
highly correlated with acculturation) was found to be a significant predictor of wife assault. More specifically, U.S.-born Latinas experienced higher rates of wife assault than their non-U.S.-born counterparts. Increased rates of partner abuse among U.S.born Latinas are consistent with other research (Firestone, Lambert, \& Vega, 1999). Garcia, Hurwitz, and Kraus (2005) examined the relationship between acculturation and reported IPV among 464 Latinas. Demographic information showed that all age groups experienced reported IPV. However, the highest prevalence (i.e., IPV or No IPV status) was among participants in the lower age groups from 15 to 19 years $(25.5 \%)$ and 20 to 24 years $(24.2 \%)$. In addition, a higher prevalence of reported IPV was found among married participants (63.1\%), homemaker (52.7\%), and worker (24.3\%). The largest proportion of participants who reported IPV were at the lowest acculturation level (59.6\%), born in Latin America (77.2\%), interviewed in Spanish (75.8\%), first generation (77.2\%), grew up only in Latin America (61.1\%), and lived in the U.S. for 1-6 years (51.3\%). Although the largest numbers of Latinas were in the lowest acculturation level, prevalence odds ratio showed that Latinas with higher acculturation levels are at a higher risk of reported IPV than Latinas with lower acculturation levels. These findings are consistent with other research that has found that higher acculturation among Latinas is associated with increased partner violence (Firestone, Lambert, \& Vega, 1999)

In contrast, lower levels of acculturation were significantly related to greater risk of partner abuse among 169 rural Mexican-American women (Champion, 1996). 
Work-related IPV 35

Similarly, Perilla, Bakeman and Norris (1994) examined both cultural and socioeconomic predictors of domestic violence among 60 immigrant Latinas. While stressors related to immigration status (e.g., lack of English proficiency, prejudice) were found to predict levels of abuse, acculturation did not. Furthermore, acculturation did not moderate the relationship between stressors related to immigration and levels of abuse.

Caetano, Schafer, Clark, Cunradi and Raspberry (2000) examined the relationship between acculturation and IPV among 527 Latino couples. Participants' scores on an acculturation scale were divided into three groups representing low, medium, and high. The findings indicate that male-to-female partner violence was highest among men in the medium acculturation group. In addition, rates of different types of IPV were slightly higher among men in the medium acculturation group. Across all three acculturation groups, the observed differences were not statistically significant. On the other hand, female-to-male partner violence was significantly two times higher among women in the medium acculturation group than among women in the other two acculturation groups (i.e., low and high). Couples with the highest level of risk for IPV were those with both partners in the medium acculturation group. The next at-risk groups were couples with at least one partner in the medium acculturation group. However, a follow-up study by Caetano, Ramisetty-Mikler, and McGrath (2004) using the same data did not support the earlier findings. In fact, only one finding was significant which suggested that couples in which there was a mixture of 
partners with high and medium level of acculturation were at less risk for male-tofemale partner violence than couples in which both partners were low in acculturation. More research is needed to better understand the discordance between partners' acculturation levels.

In summary, research on the relationship between acculturation and prevalence of IPV has produced mixed findings (Caetano, Ramisetty-Mikler, \& McGrath, 2004; Caetano, Schafer, Clark, Cunradi \& Respberry, 2000; Chun \& Akutsu, 2003; Garcia, Hurwitz, \& Kraus, 2005; Jasinski, 1998; Kasturirangan, Krishnan, \& Riger, 2004; Klevens, 2007). Some studies suggest that acculturation does not protect against partner violence and in some instances may even contribute to abuse. Some of the studies that have produced contradictory findings have been criticized for inconsistent measurement. For example, some studies have used one proxy indicator (e.g., country of birth) to measure acculturation which may only partially capture the acculturation process. Despite the inconsistent findings, it is necessary to understand the impact and direction of acculturation and IPV among Latinos (Marín \& Gamba, 2003).

\section{Acculturation Stress}

Acculturation stress (i.e., acculturative stress) occurs as a result of immigrants experiencing conflict in resolving cultural differences (Hovey \& King, 1996). These cultural differences can cause an individual in the acculturation process to feel torn between their cultural values, norms, and customs and those in the new host culture (Hovey, 2000). Stress associated with the process of acculturation, rather than 
acculturation itself, may be responsible for maladaptive behaviors. In other words, the process of adapting to another culture may result in stress, which in turn may lead to the maladaptive behaviors (Williams \& Berry, 1991). For example, some research has found that acculturation stress was related to increased partner abuse among Mexican immigrant women (Firestone, Lambert, \& Vega, 1999).

Firestone, Lambert, and Vega (1999) examined the relationships between acculturation, acculturation stress, and IPV among a large sample $(\mathrm{N}=1,155)$ of women of a Mexico-origin (i.e., U.S.-born, Mexico-born) who were involved in an intimate relationship at the time of the interview. Overall for both groups, higher acculturation was associated with increased IPV. Additionally, individuals with higher levels of acculturation stress were significantly at greater risk for physical violence and intimate rape. Economic stress was positively related to acculturation stress which the authors suggest that some women were affected by both stresses of adapting culturally and economically to the U.S. (Firestone, Lambert, \& Vega, 1999).

Studies regarding group differences (i.e., U.S.-born, Mexico-born) revealed a significantly higher mean rate of IPV among U.S.-born (50\% higher) than Mexicoborn women. Highly acculturated Mexico-born women tended to perceive less stress from the acculturation process. For the Mexico-born women, acculturation stress was also inversely related to women's age, educational level, and income. However, these relationships were not statistically significant for the U.S.-born group. Economic stress was significantly associated with higher acculturation stress for the Mexico-born 
women. However, for the U.S.-born women, the economic-acculturation stress relationship was inverse and not significantly related to acculturation stress. Controlling for acculturation, socioeconomic factors, economic stress, and others factors, acculturation stress was not a significant predictor of IPV for Mexico-born women. The authors noted the importance of acculturation remaining a significant predictor of IPV independent of acculturation stress.

Caetano, Ramisetty-Mikler, Caetano Vaeth, \& Harris (2007) examined the relationships between acculturative stress, acculturation, and IPV among 387 Latino couples. With regard to examining IPV among Latinos, it is one of the few studies that has measured both acculturation stress and acculturation and have assumed that they are independent constructs. Among men, their findings indicated that higher levels of acculturation were associated with the group reporting "any violence" and the group reporting female-to-male partner violence than the group that reported "no violence". Similarly, higher levels of acculturation stress were found in the group that reported "any violence", the group that reported male-to-female violence, and the group that reported female-to-male partner violence compared to the group that reported "no violence". Among women, both acculturation and acculturation stress were significantly higher in the groups reporting "any violence" than in the group reporting "no violence". Overall, their results suggest that both acculturation and acculturation stress are associated with a higher likelihood of IPV among Latino couples. 
Work-related IPV 39

In summary, stress associated with the process of acculturation, rather than acculturation itself, has been linked to IPV. However, the findings regarding relationship between acculturation stress and IPV are not that clear and while a good case has been made to differentiate the impact of acculturation and acculturation stress on IPV, rarely have researchers examined both. As previously mentioned, Latinos and other immigrants face many barriers in their ability to adapt and navigate in their new environments. Latinos, in particular experience poor socioeconomic conditions, limited access to resources, loss of family and friends, discrimination, difficultly in language acquisition, and conflicting gender role ideologies which can result in increased levels of acculturative stress. Torres (1998) suggests that Latinos may experience conflict between the mainstream and minority cultures because the majority of Latinos choose to retain traditional ideals that are no longer as easily attainable in the U.S. because of the rapidly changing urban environment, different sociopolitical conditions, gender role ambiguities, and contradictory cultural expectations and values.

\section{Work-related Intimate Partner Violence}

Typically, IPV has been perceived and addressed by researchers as an issue that usually occurs within a domestic context. However, the occurrence of IPV and its effects carry over from the domestic context into the workplace context. Research suggests that work-related IPV is costly and poses serious risk to women's employment, health, and safety (Ridley et al. 2005). The Centers for Disease Control 
Work-related IPV 40

reported that the annual cost of IPV includes almost $\$ 900$ million in lost productivity from paid work for victims of nonfatal IPV (2003). In addition, nearly one million people are victims of violence in the workplace each year and women are 5 times more likely than men to be attacked by an intimate partner while at work (Bachman, 1994). The U.S. Department of Labor (2007) reported that homicide is the second leading cause of death for women on the job.

Researchers have begun to address the intersection of IPV and employment (Logan, Shannon, Cole, \& Swanberg, 2007; Swanberg, Logan, \& Macke, 2005; Tolman \& Wang 2005). Research has shown that work-related IPV affects productivity, work morale, absenteeism, safety and well being of all employees and affects women's relationships with coworkers, supervisors, and customers (Brownwell, 1996; Fitzgerald, Dienenemann, \& Cadorette, 1998; Mighty, 1997; Riger, Ahrens, \& Blickenstaff, 2000). It has been estimated that between $36 \%$ and $75 \%$ of employed woman who experience IPV are harassed by their abusive partners while at work (Shepard \& Pence, 1988; Swanberg, Logan, \& Macke, 2005; Taylor \& Barusch, 2004). Lloyd and Taluc (1999) found women who had experienced IPV in their adult relationships, were more likely to experience periods of unemployment, increased turnover, and experience physical and mental problems.

Among women who experience IPV, some research has shown that employment plays a critical role in the lives of these women. For example, Rothman, Hathaway, Stidsen, and de Vries (2007) conducted in-depth interviews with 21 
Work-related IPV 41

employed women who were recruited from their employer's affiliated Employee Assistance Program (EAP). This is important to note because most studies that examine the effects of workplace-related IPV on women draw samples from women's shelters and/or welfare programs and may differ from women experiencing less severe forms of work-related IPV. For example, research suggests that victims of IPV from women's shelters and welfare programs may not be representative of employed victims because they typically have experienced severe forms of IPV which have resulted in their need to flee and/or be underemployed (Rothman et al., 2007). Therefore, it is possible that the frequency and severity of work-related IPV may be higher for these types of women than women who are not on welfare or use women's shelters. Overall, the authors identified six ways in which employment played a positive role in their lives; by (1) improving their finances (e.g., income played a crucial factor in their ability to leave their abusive partners), (2) promoting physical safety (e.g., employer's commitment to their employees' safety and well-being), (3) increasing self-esteem, (4) improving social connectedness (e.g., reduced social isolation), (5) providing mental respite (e.g., employment as a distraction from the problems at home), and (6) providing motivation (e.g., employment provides a sense of purpose in life).

What follows next is a brief review of literature based on victim's reports, which is necessary to include for two reasons. First, there is a paucity of research that examines workplace IPV from men's reports and more information is needed. Second, 
a considerable amount of knowledge, based on victim's reports, has been produced which complements our understanding of the phenomenon.

Ridley, Rioux, Lim, Mason, Houghton, Luppi, and Melody (2005) collected information from 120 women regarding the effect of IPV in the workplace of women. Researchers identified: (1) how perpetrators of IPV affected their partners in their workplace; (2) the frequency of specific IPV tactics; (3) the impact of IPV on work performance; and (4) employers responses and employee perceptions of employer responses to IPV.

Nearly the entire sample (96\%) reported that their experience of IPV affected their ability to perform their job duties, frequently over a period of months to years. The majority (98\%) reported that IPV caused difficulty concentrating on work and of those, $92 \%$ reported that their productivity was affected. In terms of the contact, $93 \%$ reported that their abuser contacted them while at work. Participants identified a number of abuse tactics and strategies that included: (1) monitoring by telephone and email (87\% and $23 \%$ reported); (2) using coworkers to get information (60\%); (3) threatening to show up at the workplace $(59 \%)$; (4) stalking in a variety of manners (57\%); and (5) showing up at the workplace (78\%). Harassment over the telephone had a marked impact on productivity. Eighty-seven percent of the participants who reported that their partners harassed them at work using the telephone on average received 5.5 calls per week. 
Other strategies and abuse tactics that interfered with their partner's employment included starting a fight before work (87\%), keeping her up late or all night (77\%), assaulting her before work (47\%), refusing to care for children or taking them to childcare/school (39\%), and hiding/taking the car keys (34\%). In addition, participants commented on a number of tactics related to disabling or interfering with the family car as a way to interfere/prevent her from going to work. Specifically, the tactics related to the car included cutting break lines, removing essential parts, flattening tires, selling or removing vehicle from use, rigging lights as a way to get her pulled over, and leaving the gas tank low.

Swanberg, Logan, and Macke's (2005) review of the literature highlighted the deleterious impact of IPV at both the individual (e.g., victimized employees; unemployment) and organizational level (e.g., higher production, health care, administration, and liability costs). Swanberg et al. found that abusive men use a variety of specific behaviors (i.e., job interference tactics) on intimate partners. Job interference tactics interfere with women's ability to get to their work, do their work, and keep their job. Some of the job interference tactics that have been identified include harassing partners at their workplace using employers' phones and vehicles, and monitoring partners during their workday (Riger, Ahrens, \& Blickenstaff, 2000; Rothman \& Perry, 2004). Swanberg et al. (2005) identified two categories of job interference tactics used by abusive men: (1) Work-related stalking; and (2) Work disruption. Work-related stalking behavior parallels general stalking behavior (e.g., 
unauthorized following and surveillance to the extent that the person's privacy is unacceptably intruded upon, and the victim fears for their safety) and can be grouped into two types: on-the-job surveillance and on-the-job harassment (e.g. unwanted and unwelcome, words, deeds, actions, gestures, symbols, or behaviors). Work disruption tactics consist primarily of actions that prevent the victim from reaching the workplace, either on time or at all, and these actions may take place in the home or off workplace premises.

Currently, the body of literature on work-related IPV has relied almost exclusively on victim's reports. The extant literature on work-related IPV is incomplete because abusive men may manipulate, monitor, stalk or harass their partners in ways that are undetected by the victim (e.g., lying about childcare arrangements to keep a partner at home rather than work). Due to this limitation in reporting, studies that assess men's reported behavior are needed to identify the entire scope of work-related IPV as perpetrated by men and its consequences on employment and performance. In particular, there is a need to understand how abusers carry out their IPV (i.e., job interference tactics), and how they affect their intimate partner's employment (Ridley et al., 2005).

Only a few studies to date have examined men's reported work-related IPV. An exploratory focus group study by Rothman and Perry (2004) was conducted with male perpetrators of IPV $(\mathrm{N}=29)$ in order to examine their impact on the workplace. Although this was a pilot study and lacked generalizability to all abusive males (i.e., 
convicted vs. non-convicted), it is one of the first to address abusive males' job interference tactics. The participant's professions ranged from craftsmen to managers with the majority representing the former. The findings indicate that the majority of the participants reported using large quantities of company time and using a range of specific work resources (e.g., company telephones, email, company vehicles, and other employees) to monitor their partners from work. Almost all participants who had access to a company phone reported using it for "checking up on" on their partners. In addition, participants who didn't have access to company phones reported using personal cell phones or pay phones to monitor their partners.

One unanticipated finding of the study was that IPV also negatively affected the men's work performance in a number of ways. Several participants made dangerous and costly mistakes because they were distracted by their IPV. The participants reported that they made costly mistakes due to emotional distress caused by their IPV behavior. For example, many reported being worried about going to prison, bothered by shame and guilt, experienced depression stemming from the separation from their children which negatively affected their ability to concentrate on work. Additional examples of decreased productivity include participants using paid work time to attend IPV-related court proceedings and incarceration and missing work for activities directly related to their IPV.

Rothman and Corso (2008) followed up on this unanticipated finding by examining the impact that propensity for abusiveness has on workplace productivity. 
Work-related IPV 46

Propensity for abusiveness was measured by a questionnaire that discriminates between men with a high versus low propensity for perpetrating IPV against a female partner. Rothman and Corso assessed the productivity of a sample of male workers ( $\mathrm{N}$ $=61$ ) by their propensity for perpetrating IPV. The majority of participants were married (87\%), college educated (90\%), and full time employees (98\%) and completed the survey online. The results suggested that propensity for perpetrating IPV was associated with workplace productivity. Specifically, controlling for demographic and socioeconomic variables (e.g., education, marital status, employment status), greater propensity for perpetrating IPV was positively associated with missing work (e.g., absenteeism) and experiencing worse productivity on the job (e.g., presenteeism). This research suggests that employed abusive men may be more likely than other employees to miss work (or portions of the workday), underperform on-the-job, make costly mistakes, ultimately creating additional costs to organizations that employ them.

\section{Summary of Literature}

Research has shown that a disproportionate number of ethnic minorities, specifically Latinos, experience higher rates of IPV than non-Latinos (Caetano, Field, Ramisetty-Mikler, \& McGrath, 2005; Ingram, 2007; Kantor, Jasinski, \& Aldorondo, 1994; Sorenson \& Telles, 1991). Some studies that examine IPV among Latinos have sought to understand the role socioeconomic status indicators and have found that group differences tend to disappear when socioeconomic status indicators were 
controlled for in analyses (Kaufman, Jasinki, \& Aldorondo, 1994). Latinos as a group face numerous barriers and sociocultural contexts that uniquely influence and shape their experience of IPV. Some researchers have acknowledged the U.S. Latino contexts and have approached the study of IPV with an understanding of the intersectionality of the social forces (e.g., poverty) that are at work in the occurrence of IPV (Carrillo \& Zarza, 2008).

Latinos by no means are a homogenous group (e.g., different immigration contexts) but they do share some common cultural values and social scripts such as familismo, machismo, and marianismo (Marín \& Marín, 1991). Many of these values and scripts have been invoked by researchers as important contexts to understand IPV in Latino communities. In addition, some research has examined the relationship between these cultural values and scripts and acculturation.

Acculturation refers to a lifelong process by which immigrants assimilate and accommodate to the majority culture's way of life (Berry, 1997). It is the continuous contact between two or more distinct groups that causes cultural change in the individual (Berry, 1997). The acculturation process is difficult for Latinos because they are faced with many challenges, such as adjusting to a new language, different customs and norms for social interactions, lifestyle changes (e.g., rural to urban), and unfamiliar rules and laws that may uniquely shape or influence their experience of IPV (Balls Organista, Organista, \& Kurasaki, 2003). 
Work-related IPV 48

The role of acculturation on IPV has been explored in various studies but it has produced mixed findings (Chun \& Akutsu, 2003; Garcia, Hurwitz, \& Kraus, 2005; Jasinski, 1998; Kasturirangan, Krishnan, \& Riger, 2004; Klevens, 2007). Some studies suggest that acculturation does not protect against partner violence and in some instances may even contribute to abuse. Some of the studies that have produced contradictory findings have been criticized for inconsistent measurement. For example, some studies have used one proxy indicator (e.g., country of birth) to measure acculturation which may only partially capture the acculturation process. Despite the inconsistent findings, researchers agree that the impact and direction of acculturation on IPV among Latinos is an important factor which needs to be better understood (Marín \& Gamba, 2003).

IPV has commonly been perceived as only occurring within a domestic context. However, the occurrence of IPV and its effects carry over from the domestic context into the workplace context. Work-related IPV is costly and poses serious risk to women's employment, health, and safety (Ridley, Rioux, Lim, Mason, Houghton, Luppi, \& Melody, 2005; Swanberg, Logan, \& Macke, 2005). More recently, there has been a growth in the literature that has examined work-related IPV. However, there are important gaps in the literature that need to be addressed. Very few studies have examined how abusive men perpetrate work-related IPV which is important because they may manipulate, monitor, stalk or harass their partners in ways that are undetected by the victim (e.g., lying about childcare arrangements to keep a partner at 
Work-related IPV 49

home rather than work). In addition, the extant literature on work-related IPV has largely ignored the experience of ethnic minorities (Logan, Shannon, Cole, \& Swanberg, 2007; Ridley, Rioux, Lim, Mason, Houghton, Luppi, \& Melody 2005; Rothman \& Perry, 2004; Rothman \& Corso, 2008; Rothman, Hathaway, Stidsen, \& de Vries, 2007; Swanberg \& Macke, 2006; Tolman \& Wang, 2005). Many factors suggest that rates and forms of IPV might be different among other racial and ethnic groups (Klevens, 2007).

\section{Research Questions and Hypotheses}

The aim of this study is to examine the role of acculturation on work-related IPV among Latinos. Specifically, this study seeks to assess and report the lifetime rates of work-related IPV behaviors and tactics among Latinos enrolled in batterer intervention programs and to assess the relationship between acculturation and workrelated IPV. In addition, this study assesses the relationship between several proxy measures of acculturation that tap other dimensions of the concept and work-related IPV. The first research question is descriptive in nature, whereas the other research questions will be followed by testable hypotheses.

1. What are the forms of work-related IPV that occur among men enrolled in batterer intervention programs?

There is a lack of research that examines men's reports of work-related IPV. Even fewer studies examine information regarding the forms of work-related IPV among ethnic groups. As a result, forms of work-related IPV among Latinos are 
Work-related IPV 50

largely unknown. A previous study of work-related IPV among Latinos found several abuse tactics that were not previously found in the literature (Galvez, Mankowski, McGlade, Ruiz, \& Glass, 2011). This research question will present information regarding the various forms of work-related IPV tactics.

2. What is the lifetime prevalence of work-related IPV among Latino men enrolled in batterer intervention program?

Little empirical data exist on men's reports of work-related IPV. As a result, rates of work-related IPV among abusive men are unknown. Comparatively, given that approximately $25 \%$ of women reported that they were raped and/or physically assaulted by a current or former spouse, cohabitating partner, or date at some point in their life (Tjaden \& Thoennes, 2000), significant rates of work-related IPV should be expected. Furthermore, most published reports on the relationship between ethnicity and IPV in the general population suggest that Latinos have higher rates of IPV than Whites (Caetano, Ramisetty-Mikler, Caetano Vaeth, \& Harris, 2007; Caetano, Schafer, \& Cunradi, 2001). However, research also indicates that differences between ethnic groups tend to disappear once socioeconomic factors are considered (Caetano, Cunradi, Clark, \& Schafer, 2000; Coker, Smith, McKeown, \& King, 2000; Kantor, Jasinski, \& Aldorondo, 1994). In light of this important relationship between IPV and socioeconomic status indicators, I predict:

2a. What is the relationship between socioeconomic status indicators (e.g., income, education, employment status) and work-related IPV? 
- Income will be negatively associated with work-related IPV.

- Education will be negatively associated with work-related IPV.

- Employment status will be negatively associated with work-related IPV.

2b. Do socioeconomic status indicators (e.g., income, education, employment status) moderate the relationship between ethnicity and work-related IPV?

- Socioeconomic status indicators (e.g., income, education, employment status) will moderate the relationship between ethnicity and work-related IPV. Specifically, I predict that the interaction between ethnicity and socioeconomic status indicators will be significant. The relationship between ethnicity and workrelated IPV depends on socioeconomic indicators. For Latinos, the relationship between ethnicity and work-related IPV is negative, such that work-related IPV decreases as socioeconomic status indicators increases. For non-Latinos, the relationship between ethnicity and work-related IPV is negative, suggesting that the work-related IPV decreases as socioeconomic status indicators increases.

3. What is the relationship between four single item indicators of acculturation and a language preference acculturation scale? 
Work-related IPV 52

Researchers have argued that the inconsistent findings of studies that examine the role of acculturation on IPV among Latinos are partly due to inconsistent measurement of acculturation (Caetano, Ramisetty-Mikler, \& McGrath, 2004; Chun \& Akutsu, 2003; Jasinski, 1998). For example, measures of acculturation have varied from using multiple-item scales tapping acculturation as a linear process (e.g., Caetano, Schafer, Clark, Cunradi, \& Raspberry, 2000), multiple-item scales tapping acculturation as multidimensional process (e.g., Cuellar, Arnold, \& Maldonado, 1995), language preference-based scales (e.g., Marín, Sabogal, Marín, Otero-Sabogal, \& Perez-Stable, 1987), single item proxy measures of immigration status (e.g., Sorenson \& Telles, 1991), or language preference and country of birth/national origin (e.g., Jasinski, 2001; Kantor et al., 1994; Sorenson \& Telles, 1991).

3. What is the relationship between four single item indicators of acculturation and a language preference acculturation scale?

- Country of birth will be a unique predictor of acculturation. That is, U.S. born (vs. non-U.S. born) is associated with higher acculturation.

- Language of survey will be a unique predictor of acculturation. That is, English language (vs. Spanish language) is associated with higher acculturation.

- Age-of-arrival will be a unique predictor of acculturation. That is, younger age (vs. older age) is associated with higher acculturation. 
Work-related IPV 53

- Number of years in the U.S. will be a unique predictor of acculturation. That is, greater number of years in the U.S. (vs. lower number of years in the U.S.) is associated with higher acculturation.

Based on these findings, in addition to examining acculturation measured by a language preference scale, proxy indicators of acculturation will be examined in subsequent analyses.

4. What is the role of acculturation on work-related IPV among Latinos enrolled in batterer intervention programs?

The limited number of studies that have examined the role of acculturation in IPV among Latinos have produced inconsistent findings (Chun \& Akutsu, 2003; Garcia, Hurwitz, \& Kraus, 2005; Jasinski, 1998; Kasturirangan, Krishnan, \& Riger, 2004; Klevens, 2007). For example, some studies have indicated that more acculturated Latinos have higher rates of IPV than the less acculturated (Caetano, Schafer, Clark, \& Cunradi, 1998; Jasinski, 2001; Kantor, Jasinski, \& Aldorondo, 1994). Whereas a smaller number of studies have found that low acculturation may be related to higher rates of IPV (Caetano, Ramisetty-Mikler, \& McGrath, 2004; Champion, 1996; Sabogal, Marín, Otero-Sabogal, Marín, \& Perez-Stable, 1987).

4a. What is the relationship between socioeconomic status indicators and acculturation?

- Socioeconomic status indicators (e.g., income, education, employment status) will be positively associated with acculturation. 
Work-related IPV 54

4b. What is the role of acculturation on work-related IPV among Latinos enrolled in batterer intervention programs?

- Acculturation will be positively associated with work-related IPV.

4c. Does socioeconomic status moderate the relationship between acculturation and work-related IPV?

- Socioeconomic status indicators (e.g., income, education, employment status) will moderate the relationship between acculturation and work-related IPV. Specifically, I predict that the interaction between acculturation and socioeconomic status indicators will be significant. The relationship between acculturation and work-related IPV depends on socioeconomic indicators. For Latinos, the relationship between acculturation and work-related IPV is negative, such that work-related IPV decreases as socioeconomic status indicators increases.

5. What role do levels of acculturation among couples play in work-related IPV?

The incongruity of acculturation status between couple members has not been extensively researched. Theoretically, it is plausible that certain acculturation patterns, in particular, mismatched levels (e.g., lower and higher), may increase IPV because of different expectations regarding roles within the couples (Caetano, Schafer, Clark, Cunradi, \& Raspberry, 2000). Caetano et al. (2000) found that Latino couples with at least one partner in the medium acculturation group were three times more likely to 
experience male-to-female partner violence than couples whose partners are both low in acculturation. However, a follow-up study by Caetano, Ramisetty-Mikler, \& McGrath (2004) showed that couples in which there was a mixture of high and medium levels of acculturation were at less risk for male-to-female partner violence than couples in which both partners were low in acculturation. This research question will explore the incongruity of acculturation status between couple members and work-related IPV. 
Work-related IPV 56

\section{CHAPTER III}

\section{Methodology}

\section{Study Context}

The current study is part of an ongoing five-year NIH/National Institute of Nursing Research funded project entitled "Community Partnered Response to Intimate Violence". Consistent with ecological models that propose understanding IPV from different levels of analysis and contexts (Edleson \& Tolman, 1992), information was sought from various sources in order to understand the different facets of work-related IPV. Researchers on this project conducted a series of interviews, focus groups, and surveys from employers, employed victims of IPV and their co-workers, and men enrolled in BIPs and their group facilitators. The aim of the project is to develop and evaluate an Oregon-based community-partnered intervention for employed immigrant and U.S.-born victims of IPV within a workplace context.

IPV interventions focused on immigrant women in the workplace context are sound because research has indicated that ethnic minority women who experience IPV face many barriers (e.g., language) to accessing formal healthcare and social services (Bauer, Rodriguez, Quiroga, \& Flores-Ortiz, 2000). In particular, Latinas who experience IPV underutilize formal services in the legal and healthcare systems because of specific cultural barriers (e.g., low acculturation) (Lipsky, Caetano, Field, \& Larkin, 2006). Efforts directed at the workplace may be more successful at providing assistance and resources than formal services that currently exist. As a result 
Work-related IPV 57

of the larger project's focus on eliminating health disparities, both Latinos and Latinas were purposefully over-sampled in this project and in this proposed study. Sample and Setting

A cross-sectional survey was administered at nine community-partnered batterer intervention programs (BIPs) throughout the state of Oregon from May 2006 to December 2006. Eligible participants were men enrolled in English or Spanish language BIPs in rural and urban communities in Oregon. Study participants $(\mathrm{n}=198)$ were adult men (18 years and older) primarily court mandated to participate in a BIP. Only one man declined to participate in the study. The study sample consisted of heterosexual men as evident in no reports of same sex partners. The study was reviewed and approved by the Portland State University Institutional Review Board prior to initiating the study.

\section{Procedure}

Using a directory of batterer intervention programs in Oregon developed by Mankowski et al. (2004), the author identified all batterer intervention programs that offered Spanish/bilingual groups for the first phase of data collection (e.g., focus groups) for the larger grant project. For this proposed study, which uses data from the second phase, the author contacted all 17 programs that had been previously identified in the first phase of data collection. In some cases, some group facilitators had already participated in the first phase of data collection and they became the principal contact for the survey data collection. In addition, some programs had either stopped offering 
services, had few Latino clients, or had few groups and thus were not included in this study. The author recruited participants by contacting group facilitators and program directors via mail correspondence, email, telephone, and face-to-face meetings. After a series of verbal/written communication and face-to-face meetings with personnel (e.g., program directors, group facilitators) from different programs, a total of 10 programs allowed us access to their clients ( 3 programs had multiple sites). The author recruited English- and Spanish-speaking adult men enrolled in BIP. The author attended one of the regularly scheduled weekly meetings at the different BIP sites. During these meetings, the author presented information about the study to the men in Spanish and English (sometimes both English and Spanish depending on the language preference of the participant). The author invited the men to participate in an anonymous selfadministered survey which was to be completed on-site during one of their regularly scheduled BIP group meetings. The group facilitators were not present during any of the survey sessions. However, the facilitators were present in the BIP offices, and often in the group before or after the survey was administered, often to briefly explain the research or to introduce you to the group. This helped to ensure that participants were not pressured to participate in the study by their group facilitators. The men were informed that refusal to participate would have no effect on their relationship with the BIP. Following the presentation of the study, men who were interested in participating in the survey provided verbal informed consent in either English or Spanish by the research team. 
Work-related IPV 59

A cross-sectional design was used in the proposed study because the main purpose was to assess the lifetime prevalence of work-related IPV. The survey was self-administered and participants answered them in a group setting at a BIP. The survey sessions took place in their regular meeting rooms. All participants completed the survey anonymously and had the option of answering an English or Spanish language version of the survey. Additionally, during data collection, the author became concerned that not all men in the BIPs had the adequate literacy level needed to read and self-administer the survey. Therefore, a new method of data collection was developed that enables respondents with a wide range of literacy levels to selfadminister surveys by privately listening to the questions in English or Spanish on an iPod® digital device (Galvez, Mankowski, Braun, \& Glass, 2009). Approximately $59 \%(\mathrm{n}=117)$ of the entire sample had the option of using the iPod format $(41 \% \mathrm{had}$ taken the survey in the paper and pencil versions before the device was developed) and $5.1 \%$ of these men $(n=6)$ opted to use it instead of the paper and pencil version. Both formats of the survey took approximately 45 minutes to complete and differences between modes were not noted. Men were paid $\$ 15$ for their participation in the study. Measures

Work Abuse Survey. Work-related IPV was measured by the Work Abuse Survey (WAS; Mankowski, Galvez, Perrin, Hanson, Rollins, \& Glass, 2011). It is the first extensive survey specifically developed to assess work related IPV. A total of 107 items (98 quantitative and 9 qualitative) were developed that tap a host of work-related 
abusive behaviors (e.g., on-the-job harassment) and impacts on participants' work performance and employment outcomes. Out of the 55 items that tapped work-related abusive behaviors, a subset of the items comprised five subscales of work-related abusive behaviors and they are: (1) Threatened or actual abuse, which included threatened or actual physical or emotional abuse or violence that impedes a partner's employment. An example item is "I have threatened to hurt my partner (ex-partner) physically (e.g., slapped, hit, kicked, choked) at her job"; (2) Work interference, which included behaviors that hinder, obstruct or impede victim's employment or job performance. An example item is “I have bothered my partner's (ex-partner's) coworkers or boss with frequent calls to her work"; (3) Monitoring, which included behaviors that keep tabs on, keep an eye on, or keep the partner under surveillance (work-related). An example item is "I have followed my partner (ex-partner) to or from her job"; (4) Jealousy, which included thoughts, feelings, and behaviors that occur when a person believes a valued relationship is threatened by a partner's coworker or boss. Example item are "I have felt jealous of my partner (ex-partner) spending time with her male coworkers or boss" and "I have accused my partner (expartner) of having sex with her coworker or boss"; and (5) Control, which included behaviors that use power to direct or determine a partner's employment or work hours. Example items are "I have made my partner (ex-partner) quit her job" and "I have told my partner (ex-partner) to cut back her hours at work to spend more time at home". All subscales and items are presented in Table 1. 
Work-related IPV 61

Table 1

Five Work Abuse Survey Subscales and Items

\begin{tabular}{ll}
\hline Subscale & Item \\
\hline Control & I have told my partner (ex-partner) to quit her job. \\
I have made my partner (ex-partner) quit her job. \\
I have told my partner (ex-partner) to cut back her hours at work to spend \\
more time at home. \\
I have talked with my partner's (ex-partner's) boss about changing her \\
work schedule. \\
I have felt jealous of my partner (ex-partner) spending time with her male \\
coworkers or boss. \\
I have accused my partner (ex-partner) of having sex with her coworker or \\
boss. \\
I have been concerned with my partner (ex-partner) having a relationship \\
with men at work. \\
I have checked up on my partner (ex-partner) at her job. \\
I have followed my partner (ex-partner) to or from her job. \\
I have called my partner's (ex-partner's) job in order to check up on her. \\
I have driven my partner (ex-partner) to and from work so that I can keep \\
an eye on her. \\
I have asked a friend to keep an eye on my partner (ex-partner) while she \\
is at work. \\
I have sent email from my job to check up on my partner (ex-partner). \\
I have sent electronic messages from my job to check up on my partner \\
I have used my job's phone to check up on my partner (ex-partner). \\
partner). \\
I have used company vehicles (e.g., truck, van) to check up on my partner \\
Monitoring
\end{tabular}


Work-related IPV 62

Table 1 (cont.)

Five Work Abuse Survey Subscales and Items

\begin{tabular}{ll}
\hline Subscale & Item \\
\hline Threatened & Have you ever made your partner (ex-partner) late or miss work or \\
or Actual & interfered with her ability to get to work? Did you do any of the \\
Abuse & following? \\
& Caused her to be emotionally traumatized? \\
& Destroyed her personal property? \\
& Injured her? \\
& I have threatened to hurt my partner (ex-partner) physically (e.g., slapped, \\
& hit, kicked, choked) at her job. \\
I have physically hurt (e.g., slapped, hit, kicked, choked) my partner (ex- & \\
partner) at her job & \\
I have physically hurt (e.g., slapped, hit, punched, kicked, choked) my \\
partner (ex-partner) in front of her coworkers or boss. \\
I have threatened to hurt my partner (ex-partner) with a weapon (e.g. \\
knife, gun) at her job. \\
I have hurt my partner (ex-partner) with a weapon (e.g. knife, gun) at her \\
job. \\
I have sent threatening messages to my partner (ex-partner) at her job \\
(e.g., on a gift card). \\
I have used my company's computers or fax to send messages to my \\
partner (ex-partner). \\
I have used property at my job (e.g., gun, knife, vehicle, staple gun, \\
hammer) to hurt my partner (ex-partner).
\end{tabular}


Work-related IPV 63

Table 1 (cont.)

Five Work Abuse Survey Subscales and Items

\begin{tabular}{ll}
\hline Subscale & Item \\
\hline Work & Have you ever made your partner (ex-partner) late or miss work or \\
Interference & interfered with her ability to get to work? Did you do any of the \\
& following? \\
& Interfered with transportation \\
& Interfered with her sleep \\
Interfered with childcare arrangements & Took or hide items she needs for work \\
Refused to take care of children & \\
I have spread rumors about my partner (ex-partner) at her job. \\
I have lied about our children (e.g., child is sick, babysitter is sick) to \\
make my partner (ex-partner) miss or leave work. \\
I have bothered my partner's (ex-partner's) coworkers or boss with \\
frequent calls to her work. \\
My partner (ex-partner) has gotten in trouble at her job because I \\
frequently called her. \\
My partner (ex-partner) has gotten in trouble at work because I visited \\
her too often. \\
I have argued with my partner (ex-partner) on the phone while she was at \\
work. \\
I have called my partner's (ex-partner's) boss to complain about her. \\
\end{tabular}

The survey assessed the lifetime occurrence of many work-related IPV behaviors and used a dichotomous response format (i.e., "yes" or "no"). The authors of the survey used this type of response format because there is currently no information about the prevalence of work-related IPV behaviors. In addition, the authors wanted to assess any occurrence of work-related IPV in an effort to produce 
the least conservative prevalence estimates. Lastly, the authors wanted the response scale to be as simple as possible for the participants to use.

Categorical variables were created for each of the WAS subscales. The criterion that was used for each subscale was: Yes to one or more items. That is, reporting a "yes" to any one item in a subscale (e.g., Threatened or Actual Abuse Subscale) would count as a "yes" or the presence of the behavior(s) for that subscale. The rationale that was used to create these criteria was conceptually informed by principles used in epidemiology and public health (Gerstman, 2003). In viewing IPV as a public health problem, prevalence rates can be calculated as the percentage of a sample that report one or more instances of an IPV-related behavior(s) in a scale. Similarly, prevalence rates of IPV are calculated in the same manner for the Conflict Tactics Scale, a widely used measure of IPV behaviors (Straus, Hamby, BoneyMcCoy \& Sugarman, 1996).

The development of the scale items were initially based on analysis of transcripts of focus groups conducted with both participants and group facilitators in batterer intervention programs (Galvez, Mankowski, McGlade, Ruiz, \& Glass, in press). This method has been recommended in the literature as a way to develop surveys that specifically tap sensitive behaviors (e.g., IPV perpetration) subject to self presentation bias (O’Brien, 1993). Items were also informed by the review of prior studies that assessed perpetrators work-related IPV (Lim, Rioux, \& Ridley, 2004; 
Rothman \& Perry, 2004). Overall, the development of the survey was overseen by multidisciplinary team of subject matter experts.

The scale items were reviewed by an established group of local BIP providers during one of their regular scheduled meetings. Members read the survey and made minor corrections and recommendations (e.g., wording). After the author made the changes and presented it back to the larger team for review, the survey was pilot tested in two separate sessions with men enrolled in BIPs. Both groups of men were invited to complete the survey and to participate in a focus group to discuss the survey and make recommendations for improvement. Several minor revisions were recommended by the participants (e.g., move open-ended questions to the end of the survey). Following the revisions that were suggested, the survey items were finalized.

The validation of the items and five subscales (subset of 40 items) was initially established through two procedures; Q-sort (Stephenson, 1953) and relevancy ratings (Cunningham, Burton, Hawes-Dawson, Kington \& Hays, 1999). A Q-sort is a technique used to analyze subjectivity in a structured and statistically interpretable form and it is comparable to a factor analysis. The q-sort allows researchers to examine subjectivity in an organized manner. Subject matter experts were asked to complete a Q-sort which asked them to indicate which subscales best represented the scale items. The Q-sort procedure asked participants to indicate which of the five categories (subscales; i.e., Jealousy, Control, Monitor, Severity, \& Interference) of abuse "best represents" each of the items by placing an " $\mathrm{x}$ " in one of five labeled 
columns. Their responses resulted in further refinement of items selected for each of the five subscales. Following the Q-sort, a different group of subject matter experts completed relevancy ratings of the items to their corresponding subscales.

Respondents evaluated the relevance of each item to its corresponding subscale by rating the degree to which each statement is relevant to the scale title and description (i.e., 0 = "not at all relevant", 1 = "somewhat relevant", or 2 = "highly relevant").

Following this procedure, the scale and scale descriptions were finalized and construct validation was achieved (Mankowski, Galvez, Perrin, Hanson, \& Glass, under review).

Short Acculturation Scale. Acculturation was primarily measured by using the Short Acculturation Scale for Hispanics (SASH) developed by Marín, Sabogal, Marín, Otero-Sabogal, and Perez-Stable (1987). The original scale contains 12 items which are related to three factors; (1) Language use; (2) Media; and (3) Ethnic social relations. The response format is on a 1 to 5 point Likert scale; (1) Only Spanish; (2) Spanish better than English; (3) Both equally; (4) English better than Spanish; or (5) Only English. The internal consistency for this scale is satisfactory (Cronbach's alpha $=.92)$. A 5 -item version of the survey which focuses only on factor 1 (i.e., Language) displayed comparable reliability to the full 12 item version (Cronbach's alpha $=.90)$ (Marín, Sabogal, Marín, Otero-Sabogal, \& Perez-Stable, 1987). The total score can range from 5 (least acculturated, greater use of Spanish language) to 25 (most acculturated, greater use of English language). The current study uses the 5-item 
version which has been recommended for use among Latinos within the context of a large study where acculturation is one of many measures to be included (Marín et al.). Example items are "In general, what language(s) do you read and speak?" and "In general, what languages do you usually speak with your friends?"

In addition to assessing acculturation with a valid measure of the concept (i.e., SASH), several proxy measures were assessed in order to examine other dimensions of the concept. Items related to language preference of interviews/surveys, country of birth (U.S. born vs. Non-U.S. born), age-of-arrival, and length of time in the U.S., have all been commonly used as indicators of acculturation (Kantor, Jasinski, \& Aldorondo, 1994; Champion, 1996: Perilla, Bakeman, \& Norris, 1994; Sorenson \& Telles, 1991). Additionally, the current study examined the relationship between all of these indicators and work-related IPV.

Ethnicity was measured by a set of questions that followed the format of the 2000 U.S. Census Bureau (e.g., separate questions regarding ethnicity and race). Specifically, participants were asked "Are you Spanish/Hispanic/Latino". If participants responded "yes", they were asked to further indicate their subgroup affiliation (e.g., Mexican, Mexican American, Chicano, Puerto Rican, Cuban, Central American, South American, other Spanish/Hispanic/Latino). For those that respond "no" to this question, a subsequent question regarding participants' race was asked which included six options (e.g., White, Black or African American, Asian, American Indian or Alaska Native, Native Hawaiian or other Pacific Islander, and Other). These 
two sets of questions are important because they can capture the complexity of the make-up of Latinos in the U.S. Marín and VanOss Marín (1991) both contend that an individual may be ethnically "Hispanic" and racially Black or White at the same time. Data from the 2000 U.S. Census supports this complexity as indicated by the almost equally high rates of Latinos who identified themselves as being racially White (48\%) or some other race (42\%) (Tafoya, 2004).

Other demographic information was collected with 38 additional questions. The questions asked participants to report their age, income (e.g., 10 categories of monthly gross income), education (e.g., highest grade completed and an additional checklist of categories which included no school, GED or high school diploma, some college, vocational, 4-year college, and post baccalaureate), employment status (e.g., currently employed, working full time/part time, unemployed, inside/outside home employment, job type, union), religious affiliation, their context regarding their participation in batterer intervention programs (e.g., length of time in program, reasons for coming to program), relationship status (never married, married, widowed, divorced, separated, single living alone/with children, and single living with a partner), living contexts (e.g., number of people in household, number of children, number of children living at home, and living in own household/ with partner's family/with own family) and partner's information (e.g., gender, ethnicity, race, employment status). 
Work-related IPV 69

\section{Translation}

The survey was translated from English to Spanish using a committee approach following the procedure recommended by cross-cultural researchers (Brislin, 1986; Marín \& Marín, 1991). First, the English version was translated to Spanish by a bilingual research assistant and reviewed by the author (a bilingual graduate student). In several instances, some words were difficult to translate. In these cases, specific words and sentences were brought to meetings with the larger research team which consisted of a subgroup of bilingual and bicultural team members. Conversations took place among the researchers and meanings were discussed. In some cases, additional words that conveyed the same meaning were placed within parentheses in order to make sure that participants would understand the word(s) and meaning. For example, "paycheck" as part of an item was supplemented with the word "payment" in parentheses in the Spanish version. Following this, a back-translation process was used in which yet another bilingual research assistant translated the Spanish version back to English, which allowed for the original English and back-translated version to be compared for consistency. The author examined both versions and made minor revisions to survey items. 
Work-related IPV 70

\section{CHAPTER IV}

Results

\section{Data Cleaning}

Using SPSS version 16.0, data from all the surveys was entered into a computer by the author. The accuracy of data entry was achieved by having a research assistant enter the data from the surveys into a separate SPSS spreadsheet. Both spreadsheets were imported into MS Excel in order to compare and analyze the data for errors. Errors were double-checked with the hardcopy surveys and final changes were made to the datasets. Data cleaning was further achieved by running frequency distributions and histograms. This allowed the data to be examined for further coding or data entry errors. For example, data outside the number range of particular variables was identified and double-checked with the hardcopy surveys. The distributions of the variables were examined for outliers, skewness, and normality. As a result, no variables were transformed or not used in the analyses. In addition, the variables used for specific analyses (e.g., multiple regression) were examined and shown to adequately meet the assumptions associated with the analyses. For example, the assumption of linearity was examined by creating bivariate scatterplots of the variables of interest. A curvature in the relationship between the variables would have indicated a violation of this assumption. However, multiple regression procedures are not greatly affected by minor deviations in this assumption. In this data, the 
Work-related IPV 71

scatterplots showed linear relationships among the variable of interest and no transformations were needed or performed.

Missing Data

A missing value analysis was conducted to examine the pattern of missing data. A variable-wise missing analysis of demographic characteristics found missing values that ranged from $0 \%$ to $42.9 \%$. A sub-question regarding school type had the greatest number of missing values (42.9\%), while language spoken fluently and living arrangements did not have any missing values $(0 \%)$. Upon closer inspection, the root question regarding how many years of schooling had a lower number of missing values (3\%). It is very possible that participants skipped the sub-question because they had checked off a value for the root question and the sub-question was not numbered. The next greatest number of missing values was how many hours were worked inside the home for money (16.7\%), is your partner (ex-partner) currently employed (15.2\%), how many children living with you are step children (13.6\%), and does your partner have a driver's license (12.6\%). Other missing values for demographic items ranged from $0.5 \%$ to $9.1 \%$. To further examine whether data were missing at random, a tabulated patterns table was produced. The table indicated that there was not a pattern of missing data. That is, data were not missing for multiple variables in individual cases. 
Work-related IPV 72

\section{Descriptive Analyses}

Study participants included 198 men at least 18 years of age or older $(\mathrm{M}=$ 34.16, $S D=10.53)$. Participants had on average 10.09 years of formal education $(S D=$ 3.32). The majority of participants were employed (89.34\%). Of those that were employed, $9.30 \%$ reported being union members. The majority of participants reported working one job (81.80\%) and some participants reported having more than one job $(16.2 \%)$. The average monthly income was $5.26(S D=2.17)$ which was anchored to the category $\$ 1,501-\$ 2,000$ monthly. The majority of the participants attended a batterer intervention program (BIP) as part of their sentencing by a criminal court or the State of Oregon Department of Human Services (91.4\%). A small proportion of participants attended voluntarily (9.6\%). The mean length of participation in a BIP was 9.55 months $(S D=10.72)$.

About half of the participants reported that they were married or living with a partner $(50.53 \%)$; the remaining were either separated or divorced $(28.19 \%)$, or single or single living with children $(21.28 \%)$. In terms of racial identification, participants were instructed to choose from various categories. The majority chose Other $(43.93 \%)$ and White (38.89\%) and fewer chose Black (5.05\%), Native American (3.54\%), Hawaiian or Pacific Islander (2.53\%), and Asian (2.02\%). As previously stated, we purposely oversampled for Latinos and as a result more than half of the sample was Latino $(60.61 \%)$. Among Latinos only, the average acculturation score as measured by the language subscale of the Short Acculturation Scale (Marin, Sabogal, Marin, Otero- 
Sabogal, \& Perez-Stable, 1987) was $9.40(S D=4.16)$. In terms of context, the acculturation subscale score in this study is very similar to the score for first generation Latinos (versus the more acculturated second generation) reported in Marin et al.'s scale development paper. More than half of the participants $(53.93 \%)$ were born outside of the U.S. with most of the participants being from Mexico (84.47\%). Non-U.S. born participants had continuously lived in the U.S. for an average of 11.52 years $(S D=5.99)$. Over half of the participants' current partners or spouses were born in the U.S. (60.32\%). Of those non-U.S. born partners, $86.67 \%$ were born in Mexico. On average, partners or spouses born outside the U.S. had lived in the U.S. for 10.14 years $(S D=7.39)$. A summary of the distribution of the participants' sociodemographic characteristics (e.g., age, relationship status, ethnicity) is presented in Table 2. 
Work-related IPV 74

Table 2

Sociodemographic Characteristics of Participants $(N=198)$

\begin{tabular}{|c|c|c|}
\hline \multirow[b]{2}{*}{ Name } & \multicolumn{2}{|c|}{ Total Sample } \\
\hline & $n$ & $\%$ \\
\hline \multicolumn{3}{|l|}{ Age Group $^{a}$} \\
\hline 18 to 25 & 45 & 23.08 \\
\hline 26 to 35 & 76 & 38.97 \\
\hline 36 to 45 & 44 & 22.56 \\
\hline 46 to 55 & 21 & 10.77 \\
\hline 56 to 70 & 9 & 4.62 \\
\hline \multicolumn{3}{|l|}{ Relationship Status ${ }^{\text {b }}$} \\
\hline Never married & 24 & 12.10 \\
\hline Now married & 64 & 32.30 \\
\hline Widowed & 3 & 1.50 \\
\hline Divorced & 27 & 13.60 \\
\hline Separated & 32 & 16.20 \\
\hline Single & 20 & 10.10 \\
\hline Single, living alone/children & 8 & 0.04 \\
\hline Single, living with a partner & 42 & 21.21 \\
\hline \multicolumn{3}{|l|}{$\operatorname{Race}^{\mathrm{c}}$} \\
\hline White & 77 & 40.96 \\
\hline Black or African American & 10 & 5.32 \\
\hline Asian & 4 & 2.13 \\
\hline American Indian or Alaska Native & 7 & 3.72 \\
\hline Native Hawaiian or other Pacific Islander & 5 & 2.66 \\
\hline Other & 85 & 45.21 \\
\hline \multicolumn{3}{|l|}{ Spanish/Hispanic/Latino $^{\mathrm{d}}$} \\
\hline Not Spanish/Hispanic/Latino & 73 & 38.22 \\
\hline Mexican & 93 & 48.69 \\
\hline Mexican American & 6 & 3.14 \\
\hline Puerto Rican & 1 & 0.52 \\
\hline Cuban & 1 & 0.52 \\
\hline Central American & 14 & 7.33 \\
\hline South American & 1 & 0.52 \\
\hline Other & 2 & 1.05 \\
\hline
\end{tabular}

Note. a. Missing $=$ excludes 3 participants with no information $(1.5 \%)$; b. Total number is greater than 198 because participants could select more than one category. The mean number of relationship status categories selected was $1.09(\mathrm{SD}=0.47)$ with a range of 0 to 4; c. Missing = excludes 10 participants with no information (5.05\%); d. Missing = excludes 7 participants with no information $(3.5 \%)$. 
Individual items were aggregated to form subscales that captured each construct (e.g., acculturation). Specifically, the acculturation scale items were averaged to form a composite score. WAS items (i.e., subscales) were averaged to form an index. For both the acculturation and WAS scores, higher values reflect higher levels of each respective construct. Descriptive analyses were conducted to calculate the means, standard deviations, and internal consistencies (i.e., Cronbach's alpha) for all the constructs and variables used in the study (see Table 3).

Table 3

Summary of Means, SDs, and Internal Consistencies for each Construct

\begin{tabular}{lcccc}
\hline \multicolumn{1}{c}{ Scale } & $\begin{array}{c}\text { Number of } \\
\text { Items }\end{array}$ & $A$ & $M$ & $S D$ \\
\hline Work Abuse Scale & & & & \\
$\quad$ Control & 4 & 0.65 & 7.46 & 0.92 \\
$\quad$ Jealousy & 3 & 0.78 & 5.21 & 1.09 \\
$\quad$ Monitoring & 10 & 0.82 & 18.54 & 2.11 \\
$\quad$ Threatened or Actual Abuse & \\
$\quad$ Work Interference & 9 & 0.65 & 16.64 & 1.48 \\
Short Acculturation Scale for Hispanics & 5 & 0.87 & 9.28 & 4.19 \\
\hline
\end{tabular}

Note. a. 2 items were removed from the 11-item subscale for these analyses because the variables had zero variance: "I have hurt my partner with a weapon at her job" and "I have used property (e.g., gun, knife, vehicle, staple gun, hammer) of my job to hurt my partner". 
Work-related IPV 76

\section{Inter-construct correlations}

The relationships between constructs were calculated using a Pearson's correlation analysis. Highly correlated (e.g., $r=.80$ and above) relationships between the constructs may indicate that they may be measuring the same underlying. Pearson's correlation coefficient was examined for magnitude and direction of each bivariate relationship and tests of significance were conducted. Significant correlations between the study constructs were found and are presented in Table 4. Overall, the findings suggest significant positive relationships between all of the work-related IPV constructs and acculturation with the exception of control and acculturation, $r(180)=-$ $.08, p=.27$. The significant findings suggest that positive relationships exist between all of the work-related IPV constructs. Relationships between these constructs were anticipated given that they were all designed to tap related aspects of work-related IPV. Highly correlated (e.g., $r=.80$ and above) relationships between the constructs were not found, which could have presented multicollinearity issues in subsequent analyses. The significant correlations between acculturation and work-related IPV suggest that positive relationships exist between them. This suggests that participants that reported higher levels of acculturation tended to report higher levels of workrelated IPV. However, a significant relationship between the control subscale and acculturation was not found. One possible explanation is related to the items that make up the control subscale. All of the items focus on behaviors that involve direct attempts at getting their partners to leave their jobs (e.g., I have made my partner quit 
her job). It's possible, that regardless of acculturation, Latinos may need their partners' financial contribution that their employment provides.

Table 4

Correlation Matrix of Study Constructs

\begin{tabular}{lcccccc}
\hline & & & \multicolumn{5}{c}{$\begin{array}{c}\text { Threatened } \\
\text { or actual } \\
\text { abuse }\end{array}$} & $\begin{array}{c}\text { Work } \\
\text { interference }\end{array}$ & SASH \\
\hline Control & -- & -- & - & -- & -- & -- \\
Jealousy & $0.33^{* *}$ & -- & -- & -- & -- & -- \\
$\begin{array}{l}\text { Monitoring } \\
\text { Threatened }\end{array}$ & $0.44^{* *}$ & $0.59^{* *}$ & -- & -- & -- & -- \\
$\begin{array}{l}\text { or actual } \\
\text { abuse }\end{array}$ & $0.17^{*}$ & $0.36^{* *}$ & $0.45^{* *}$ & -- & -- & -- \\
$\begin{array}{l}\text { Work } \\
\text { interference }\end{array}$ & $0.55^{* *}$ & $0.49^{* *}$ & $0.68^{* *}$ & $0.56^{* *}$ & -- & - \\
SASH & -0.08 & $0.23^{* *}$ & $0.21^{* *}$ & $0.33^{* *}$ & $0.24 * *$ & -- \\
\hline
\end{tabular}

Note. * Correlation is significant at the 0.01 level (2-tailed). ** Correlation is significant at the 0.001 level (2-tailed), $\mathrm{SASH}=$ Short Acculturation Scale for Hispanics.

Research Question 1. What are the forms of work-related IPV that occur among men enrolled in batterer intervention programs?

This research question addresses the types of work-related abuse tactics that were reported by all of the participants. The five items with the highest percentages were related to communication with partners while at work and interfering with their 
partner's ability to get to work. Over half (53\%) of the all the participants reported "yes" to having argued with their partner (ex-partner) on the phone while she was at work. On average, 174 of the participants reported making $2.01(S D=3.23)$ calls on a daily basis to contact or try to contact their partners while at work. The range of calls that participants reported on a daily basis was from 0 to 20 . Over one-third (37\%) reported making their partner late, miss work, or interfere with their ability to get to work. Of the participants that indicated "yes" to interfering with their partner's ability to get work, participants reported causing their partner to be emotionally traumatized (46\%), injuring their partner (42\%), and interfering with their partner's sleep (35\%). There were two items in which all participants reported a lifetime response of "no". They were "I have hurt my partner (ex-partner) with a weapon (e.g. knife, gun) at her job" and "I have used property at my job (e.g., gun, knife, vehicle, staple gun, hammer) to hurt my partner (ex-partner)". Only one participant reported yes to the question "I have threatened to hurt my partner (ex-partner) with a weapon (e.g. knife, gun) at her job." Lastly, only one participant (different than the previous one) reported yes to the question "I have called my partner's (ex-partner's) boss to complain about her". Table 5 presents the item-level responses as frequencies of participants that reported "yes" to work-related IPV. 
Work-related IPV 79

Table 5

Item-level frequency of participants' responses

\begin{tabular}{llll}
\hline $\mathrm{N}$ & Yes $(\%)$ \\
\hline
\end{tabular}

1. Have you ever made your partner (ex-partner) late or miss work or interfered with her ability to get to work?

$197 \quad 73(37.1 \%)$

2. Interfered with transportation

3. Interfered with her sleep

4. Caused her to be emotionally traumatized

5. Interfered with childcare arrangements

6. Destroyed her personal property

7. Injured her

8. Took or hid items she needs for work

9. Refused to take care of children

10. I have not given my partner (ex-partner) phone messages about work from her boss/coworker.

11. I have lied about our children (e.g., child is sick, babysitter is sick) to make my partner (ex-partner) miss or leave work.

12. I have tried to stop my partner (ex-partner) from getting a driver's license.

13. I have yelled at my partner (ex-partner) at her job.

14. I have called my partner (ex-partner) insulting names at her job.

15. I have threatened to hurt my partner (ex-partner) physically (e.g., slapped, hit, kicked, choked) at her job.

16. I have physically hurt (e.g., slapped, hit, kicked, choked) my partner (ex-partner) at her job.

17. I have yelled at my partner (ex-partner) in front of her coworkers or boss.
$19240(20.8 \%)$

$191 \quad 67(35.1 \%)$

$19489(45.9 \%)$

$190 \quad 31(16.3 \%)$

$18948(25.4 \%)$

$19280(41.7 \%)$

$19219(9.9 \%)$

$19329(15.0 \%)$

$19519(9.7 \%)$

$1949(4.6 \%)$

$175 \quad 5(2.9 \%)$

$196 \quad 28(14.3 \%)$

$197 \quad 25(12.7 \%)$

$198 \quad 14(7.1 \%)$

$198 \quad 13(6.6 \%)$

$198 \quad 21(10.6 \%)$ 
Work-related IPV 80

Table 5 (cont.)

Item-level frequency of participants' responses

N Yes (\%)

18. I have called my partner (ex-partner) insulting names in front of her coworkers or boss.

$198 \quad 16(8.1 \%)$

19. My partner's (ex-partner's) coworkers or boss have overheard me threatening to hurt her.

$198 \quad 8(4.0 \%)$

20. I have physically hurt (e.g., slapped, hit, punched, kicked, choked) my partner (ex-partner) in front of her coworkers or boss.

21. I have picked up my partner's (ex-partner's) paycheck at her job.

$198 \quad 5(2.5 \%)$

22. I have bothered my partner's (ex-partner's) coworkers or boss with frequent calls to her work.

$19822(11.1 \%)$

$19833(16.7 \%)$

23. My partner (ex-partner) has gotten in trouble at her job because I frequently called her.

$19830(15.2 \%)$

24. I have yelled at my partner's (ex-partner's) boss or coworkers.

$197 \quad 10(5.1 \%)$

25. I have threatened to hurt my partner (ex-partner) with a weapon (e.g. knife, gun) at her job.

$198 \quad 1(0.5 \%)$

26. I have hurt my partner (ex-partner) with a weapon (e.g. knife, gun) at her job.

$197 \quad 0(0.0 \%)$

27. My partner (ex-partner) has gotten in trouble at work because I visited her too often.

$197 \quad 11(5.6 \%)$

28. I have checked up on my partner (ex-partner) at her job. $\quad 198 \quad 60(30.3 \%)$

29. I have followed my partner (ex-partner) to or from her job. $193 \quad 33(17.1 \%)$

30. I have argued with my partner (ex-partner) on the phone $198 \quad 104(52.5 \%)$ while she was at work. 
Work-related IPV 81

Table 5 (cont.)

Item-level frequency of participants' responses

$\begin{array}{lll}\mathrm{N} & \text { Yes (\%) }\end{array}$

31. I have called my partner's (ex-partner's) job in order to check up on her.

$197 \quad 47(23.9 \%)$

32. I have driven my partner (ex-partner) to and from work so that I can keep an eye on her.

$198 \quad 32(16.2 \%)$

33. I have sent threatening messages to my partner (expartner) at her job (e.g., on a gift card).

$198 \quad 7(3.5 \%)$

34. I have told my partner (ex-partner) to quit her job.

$198 \quad 48(24.2 \%)$

35. I have made my partner (ex-partner) quit her job.

$197 \quad 12(6.1 \%)$

36. I have told my partner (ex-partner) to cut back her hours at work to spend more time at home.

$198 \quad 38(19.2 \%)$

37. I have talked with my partner's (ex-partner's) boss about changing her work schedule.

$197 \quad 7(3.6 \%)$

38. I have called my partner's (ex-partner's) boss to complain about her.

39. I have disapproved of my partner (ex-partner) working overtime or staying late at work.

$198 \quad 41(20.7 \%)$

40. I have tried to stop my partner (ex-partner) from spending extra time with her coworkers.

$197 \quad 29(14.7 \%)$

41. I have asked my partner's (ex-partner's) coworkers or $\quad 198 \quad 8(4.0 \%)$ boss to keep an eye on her while she is at work.

$1961(0.5 \%)$ 
Work-related IPV 82

Table 5 (cont.)

Item-level frequency of participants' responses

$\begin{array}{lll}\mathrm{N} & \text { Yes }(\%)\end{array}$

45. I have spread rumors about my partner (ex-partner) at her $198 \quad 5(2.5 \%)$ job.

46. I have sent email from my job to check up on my partner (ex-partner).

$197 \quad 5(2.5 \%)$

47. I have sent electronic messages from my job to check up on my partner (ex-partner).

$198 \quad 10(5.1 \%)$

48. I have used my company's computers or fax to send messages to my partner (ex-partner).

$198 \quad 11(5.6 \%)$

49. I have used my job's phone to check up on my partner (ex-partner).

$198 \quad 56(28.3 \%)$

50. I have asked one of my coworkers to keep an eye on my $\quad 197 \quad 9(4.6 \%)$ partner (ex-partner).

51. I have used company vehicles (e.g., truck, van) to check $198 \quad 19(9.6 \%)$ up on my partner (ex-partner).

52. I have used my job's computers or phone to gain information about my partner (ex-partner).

$198 \quad 19(9.6 \%)$

53. I have used property at my job (e.g., gun, knife, vehicle, $198 \quad 0(0.0 \%)$ staple gun, hammer) to hurt my partner (ex-partner).

54. I have used job resources (e.g., coworkers, computers, phones) to gain information about my partner (ex-partner).

55. I have learned from my coworkers or boss different ways of abusing or controlling my partner (ex-partner).

$198 \quad 18(9.1 \%)$

$198 \quad 14(17.1 \%)$ 
Work-related IPV 83

Research Question 2. What is the lifetime prevalence of work-related IPV among men enrolled in batterer intervention program?

A contingency table (cross tabulation) was created to examine the relationship between race and ethnic subgroup affiliation and reported work-related IPV subscales. As previously discussed, bivariate variables were created for the WAS subscales using the following rules: if a participant responded "no" to all items in the subscale, they were scored 0 ("no to all") and if a respondent responded "yes" to one or more items they were scored 1 ("yes to one or more"). The data for the race categories and the WAS subscales are presented in Table 6. The data for the ethnic subgroup affiliation and the WAS subscales are presented in Table 7.

To evaluate the relationship between the race categories and the WAS subscales, a Fisher's exact test was conducted ${ }^{1}$. The results suggested that the differences were not statistically significant for four (i.e., Control, Jealousy, Monitoring, Work interference) out the five subscales. However, there was a significant relationship between Threatened or actual abuse subscale and race, $p=$ 0.001, Fisher's exact test.

Regarding the relationship between ethnic subgroup affiliation and the WAS subscales, the Fisher's exact test results suggest significant differences for four out of the five subscales. There was a significant relationship between Threatened or actual abuse subscale and ethnic subgroup affiliation, $p=0.0001$, Fisher's exact test,

\footnotetext{
${ }^{1}$ Chi square analyses were originally planned at the proposal phase. However, cell counts were less than 5 which violated assumptions for the test.
} 
Interference and ethnic subgroup affiliation, $p=0.006$, Fisher's exact test, and Monitor and ethnic subgroup affiliation, $p=0.0009$, Fisher's exact test, and Control and ethnic subgroup affiliation, $p<0.05$, Fisher's exact test. Lastly, Jealousy and ethnic subgroup affiliation was not significant, $p=.07$, Fisher's exact test. Follow-up analyses will focus on examining re-categorized data. 
Work-related IPV 85

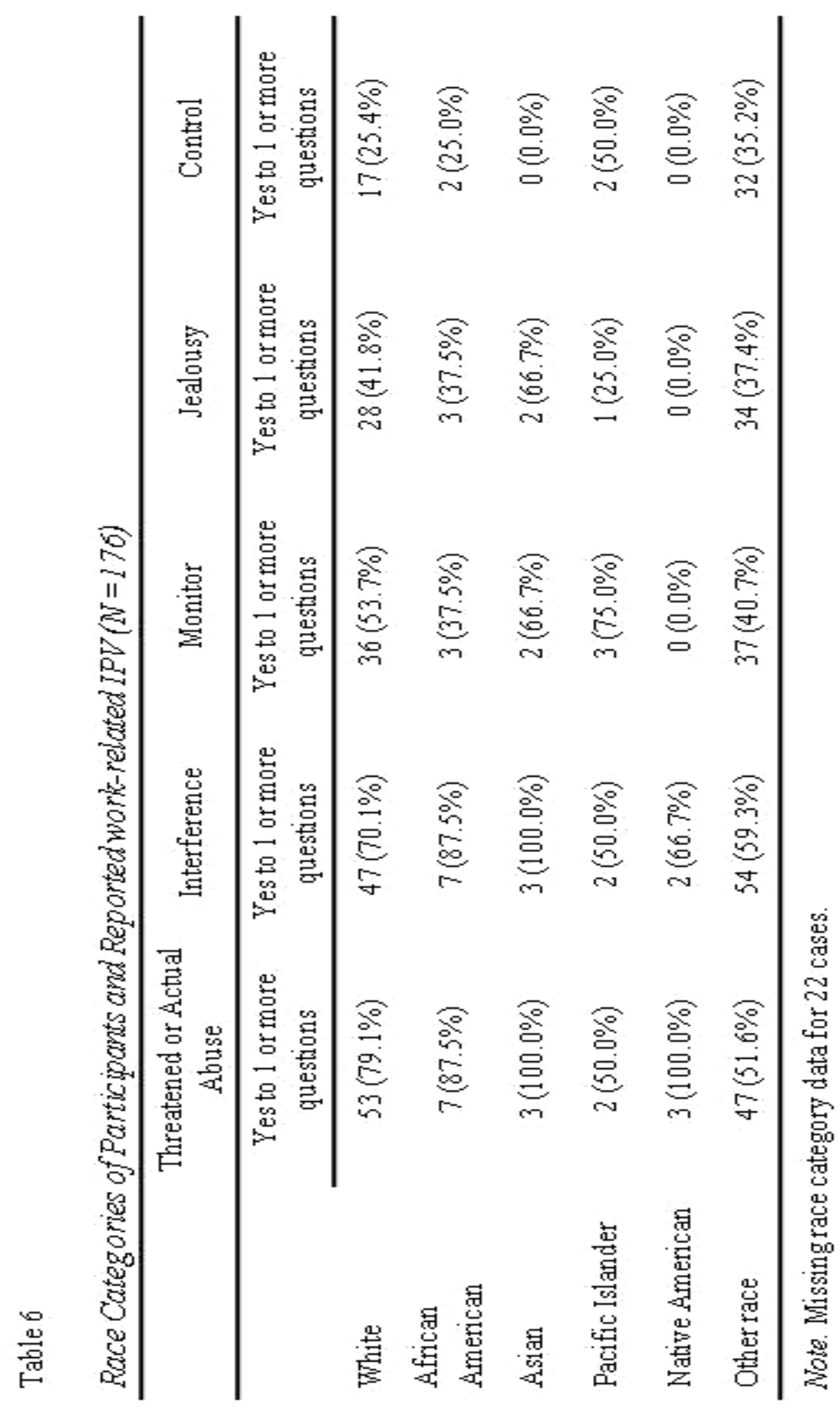


Work-related IPV 86

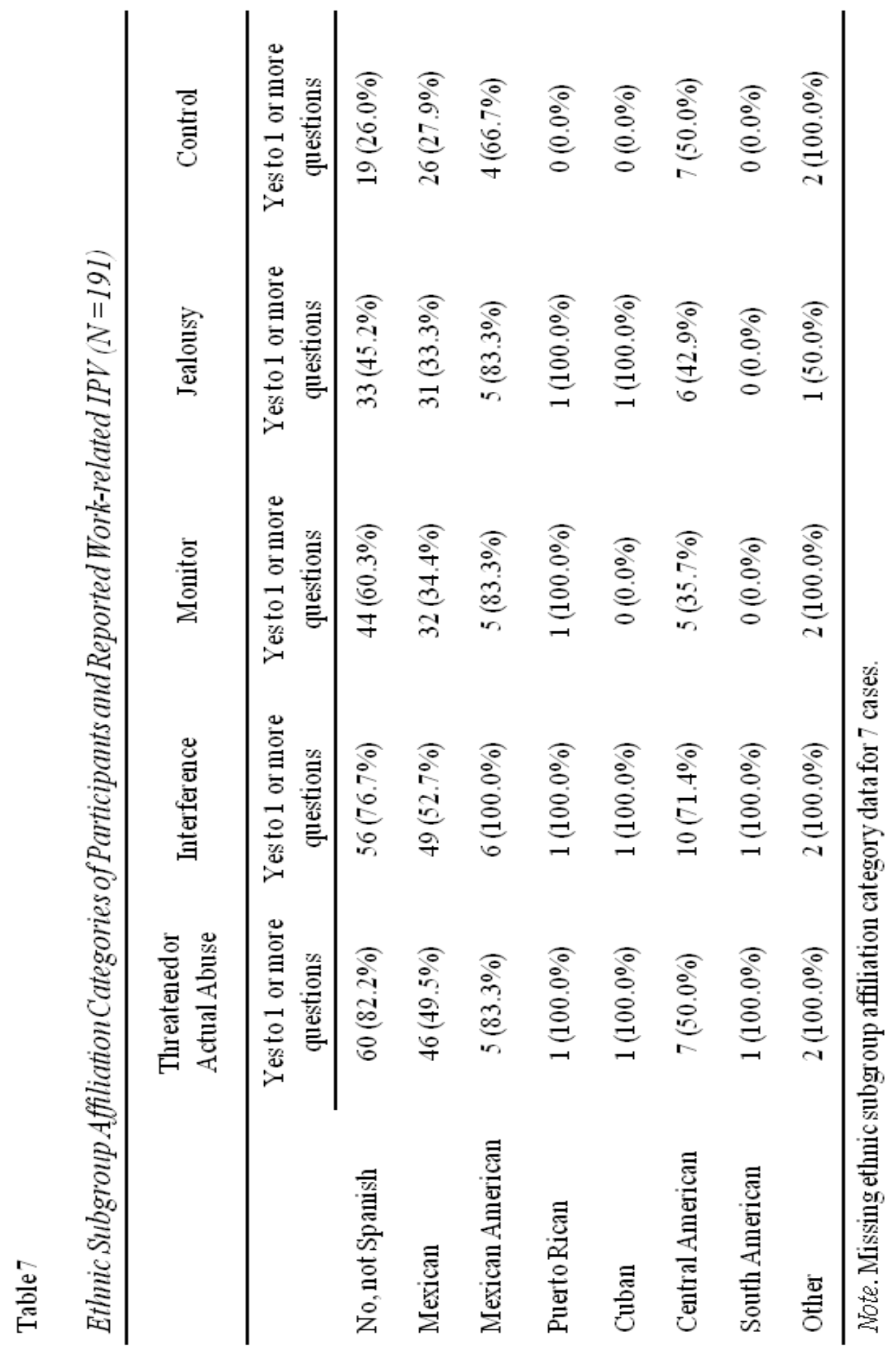


In order to clarify previous research that establishes a relationship between ethnicity and work-related IPV, two groups were created in order to examine the role of socioeconomic factors in this relationship. Due to the small number of Latino subgroups which limit separate subgroup analyses, participants were classified into either the Latino or non-Latino groups in order to examine possible differences among Latinos and non-Latinos and the role of socioeconomic status. For Latinos, the criteria for classifying group membership depended on responses to the item "Are you Spanish / Hispanic / Latino?" For example, if a participant responded "no" to this item they were classified as non-Latino.

The analyses indicated that the majority $(84 \%)$ of the participants in the Latino group were of Mexico-origin. For the non-Latino group, the majority (73\%) were White and of U.S.-origin. Since one particular Latino subgroup constitutes the majority of Latinos in the sample, the name of the Latino group variable was changed to reflect the specific subgroup (i.e., Mexican) and other Latino subgroup participants were not included in further analyses. Likewise, since one particular non-Latino subgroup constitutes the majority of non-Latinos in the sample, the name of the nonLatino group variable was changed to reflect the specific subgroup (i.e., White) and other non-Latino subgroup participants were not included in further analyses. Following this reclassification, the samples size for both Mexican and White participants were 100 and 57, respectively. 
Work-related IPV 88

Using the new classification, a contingency table (cross tabulation) was created to examine the entire sample $(\mathrm{N}=157)$ by the group of participants (i.e., Mexican, White) and reported work-related IPV. Specifically, these analyses evaluated all 5 WAS subscales and the overall work-related IPV variable. An overall work-related IPV variable was created by aggregating all 5 WAS subscales. In addition, a bivariate overall work-related IPV variable was creating by examining the frequency distribution and a histogram. The histogram clearly indicated a bimodal distribution. The value in between the distinct peaks was inspected and the bivariate variable for overall work-related IPV was created following the rule: if participant responded "yes" to 0 to 4 items, they were scored 0 ("No") and if respondents responded "yes" to 5 or more items they were scored 1 ("Yes"). This rule only applies to the overall workrelated IPV variable whereas the other subscales followed the yes to one or more items rule. The findings are presented in Table 8 .

In order to evaluate group differences and work-related IPV, a chi-square test of independence was conducted. The findings indicate that there were statistically significant relationships between the groups of participants (i.e., Mexican, White) and the Threatened or Actual Abuse subscale, $\chi^{2}(1)=12.75, p<.001$, Interference subscale, $\chi^{2}(1)=6.46, p=.01$, Monitor subscale, $\chi^{2}(1)=8.71, p=.003$, and overall work-related IPV, $\chi^{2}(1)=9.02, p=.003$. The relationship between the groups of participants and the Jealousy subscale was not significant, $\chi^{2}(1)=1.95, p=.16$. Lastly, 
the relationship between the groups of participants and the Control subscale was not significant, $\chi^{2}(1)=0.06, p=.79$.

Table 8

Mexican and White Participants and Reported Work-related IPV $(N=157)$

\begin{tabular}{|c|c|c|c|c|c|c|}
\hline & $\begin{array}{c}\text { Threatened } \\
\text { or Actual } \\
\text { Abuse } \\
\text { Subscale }\end{array}$ & $\begin{array}{c}\text { Interference } \\
\text { Subscale }\end{array}$ & $\begin{array}{l}\text { Monitor } \\
\text { Subscale }\end{array}$ & $\begin{array}{l}\text { Jealousy } \\
\text { Subscale }\end{array}$ & $\begin{array}{l}\text { Control } \\
\text { Subscale }\end{array}$ & $\begin{array}{l}\text { Overall } \\
\text { Work- } \\
\text { related } \\
\text { IPV }\end{array}$ \\
\hline & $\begin{array}{l}\text { Yes to } 1 \text { or } \\
\text { more } \\
\text { questions }\end{array}$ & $\begin{array}{l}\text { Yes to } 1 \text { or } \\
\text { more } \\
\text { questions }\end{array}$ & $\begin{array}{l}\text { Yes to } 1 \\
\text { or more } \\
\text { questions }\end{array}$ & $\begin{array}{l}\text { Yes to } 1 \\
\text { or more } \\
\text { questions }\end{array}$ & $\begin{array}{l}\text { Yes to } 1 \\
\text { or more } \\
\text { questions }\end{array}$ & $\begin{array}{l}\text { Yes to } 5 \\
\text { or more } \\
\text { questions }\end{array}$ \\
\hline Mexican & $\begin{array}{c}52 \\
(52.0 \%)\end{array}$ & $\begin{array}{c}55 \\
(55.0 \%)\end{array}$ & $\begin{array}{c}37 \\
(37.0 \%)\end{array}$ & $\begin{array}{c}36 \\
(36.0 \%)\end{array}$ & $\begin{array}{c}30 \\
(30.0 \%)\end{array}$ & $\begin{array}{c}40 \\
(40.0 \%)\end{array}$ \\
\hline White & $\begin{array}{c}46 \\
(80.7 \%)\end{array}$ & $\begin{array}{c}43 \\
(75.4 \%)\end{array}$ & $\begin{array}{c}35 \\
(61.4 \%)\end{array}$ & $\begin{array}{c}27 \\
(47.4 \%)\end{array}$ & $\begin{array}{c}16 \\
(28.1 \%)\end{array}$ & $\begin{array}{c}37 \\
(64.9 \%)\end{array}$ \\
\hline
\end{tabular}

Note. Only overall work-related IPV uses yes to 5 or more questions criteria.

In order to follow-up on the previous analyses, an independent $t$-test was conducted to determine whether there was a difference in reported work-related IPV between Mexican and White participants. An independent $t$-test was used to determine whether there was a difference in between mean score of overall work-related IPV between Mexican and White participants. This test revealed a significant difference, $t(155)=2.57, p=.01$, with White participants having significantly higher mean overall work-related IPV scores than Mexican participants (White participants, $M=$ 
Work-related IPV 90

7.61, $S D=6.21$; Mexican participants, $M=5.04, S D=5.93$ ). Furthermore, an independent $t$-test was conducted to examine differences in work-related IPV subscales scores. Four of the five subscales were significantly different. Specifically, the findings revealed a significant difference between Jealousy subscale scores, $t(94.42)=2.29, p=.02$, with White participants having significantly higher mean Jealousy subscale scores than Mexican participants (White participants, $M=1.05, S D$ $=1.23$; Mexican participants, $M=0.62, S D=0.95)$. The findings revealed a significant difference between Threatened or Actual Abuse subscale scores, $t(155)=$ 3.22, $p=.002$, with White participants having significantly higher mean Threatened or Actual Abuse subscale scores than Mexican participants (White participants, $M=$ $1.84, S D=1.59$; Mexican participants, $M=1.05, S D=1.42$ ). The findings revealed a significant difference between Monitor subscale scores, $t(155)=2.38, p=.02$, with White participants having significantly higher mean Monitor subscale scores than Mexican participants (White participants, $M=1.95, S D=2.22$; Mexican participants, $M=1.14, S D=1.94)$. The findings revealed a significant difference between Interference subscale scores, $t(155)=2.00, p<.05$, with White participants having significantly higher mean Interference subscale scores than Mexican participants (White participants, $M=2.37, S D=2.34$; Mexican participants, $M=1.63, S D=2.15$ ). Lastly, there was no difference between Control subscale scores, $t(146.13)=1.41, p=$ .20 (White participants, $M=0.40, S D=0.73$; Mexican participants, $M=0.60, S D=$ 1.01). 
Follow-up analyses were conducted to explore the possibility that differences in reported work-related IPV between the ethnic groups were related to access to workplace resources. It is possible that White participants may have more access to work-related resources such as phones, vehicles, and email, and tools and equipment than Mexican participants. Chi square analyses were conducted and the findings indicate that there were statistically significant relationships between the groups of participants (i.e., Mexican, White) and access to email, $\chi^{2}(1)=33.45, p<.001$, access to phone, $\chi^{2}(1)=13.86, p<.001$, access to vehicle, $\chi^{2}(1)=11.53, p=.001$, and access to tools and equipment, $\chi^{2}(1)=10.10, p=.001$. White participants reported higher percentages of access to email, phones, vehicles, and equipment and tools at their work than Mexican participants.

2b. Socioeconomic status indicators (e.g., income, education, employment status) will be negatively associated with work-related IPV.

A multiple regression analysis was conducted to examine the relationship between overall work-related IPV and indicators of socioeconomic status (e.g., income, education, employment status). Table 9 summarizes the descriptive statistics and analysis results. The three predictor model was significant and able to account for $9.0 \%$ of the variance in overall work-related IPV, $F(3,145)=4.86, \mathrm{p}=.003$. Contrary to what was hypothesized, two of the three socioeconomic status indicators are positively and significantly correlated with the criterion, indicating that those who reported higher income tend to have higher overall work-related IPV scores. In 
addition, those that reported more education (number of years in school) tend to have higher overall work-related IPV scores. As hypothesized, employment status (coded as $0=$ not employed and $1=$ employed) is negatively and significantly correlated with overall work-related IPV scores, indicating that unemployed participants have higher overall work-related IPV scores. And while each of the predictor variables had a significant $(\mathrm{p}<.05)$ correlation with overall work-related IPV, only income and employment status had significant $(\mathrm{p}<.05)$ partial effects in the full model. Specifically, income had a significant positive regression weight, indicating that participants with higher income had higher overall work-related IPV scores, after controlling for the other variables in the model. Employment status had a significant negative weight, indicating that after accounting for education and income, unemployed participants were expected to have higher overall work-related IPV scores. Although education was the single best predictor, it had little to offer in the context of the income and employment status and did not contribute to the model. 
Work-related IPV 93

Table 9

Multiple Regression for Socioeconomic Status Indicators on Work-related Intimate partner Violence $(N=156)$

\begin{tabular}{|c|c|c|c|c|c|}
\hline \multirow[b]{2}{*}{ Variable } & \multirow[b]{2}{*}{ Mean } & \multirow[b]{2}{*}{ SD } & \multirow[b]{2}{*}{$\begin{array}{c}\text { Correlation with } \\
\text { WIPV }\end{array}$} & \multicolumn{2}{|c|}{$\begin{array}{c}\text { Multiple Regression } \\
\text { Weights }\end{array}$} \\
\hline & & & & b & $\beta$ \\
\hline WIPV & 6.03 & 6.28 & -- & & \\
\hline Education & 9.85 & 3.17 & $0.22 * *$ & 0.27 & 0.14 \\
\hline Income $^{\mathrm{a}}$ & 5.17 & 2.08 & $0.19 * *$ & $0.51 *$ & 0.17 \\
\hline $\begin{array}{l}\text { Employment } \\
\text { status b }\end{array}$ & 0.91 & 0.28 & $-0.17 *$ & $-3.79 *$ & -0.17 \\
\hline
\end{tabular}

Note. Employed, $\mathrm{n}=142$. Not employed, $\mathrm{n}=14$. Missing data, $\mathrm{n}=1$. ${ }^{* *}$ Correlation is significant at the 0.01 level (2-tailed). * Correlation is significant at the 0.05 level (2tailed). WIPV $=$ Work-related intimate partner violence. a. Corresponds to $5=\$ 1,501$ and $\$ 2,000$ a month and $6=\$ 2,001$ and $\$ 2,500$ a month. b. Coded as $0=$ not employed and $1=$ employed.

2c. Does socioeconomic status (e.g., income, education, employment status) moderate the relationship between ethnicity and work-related IPV?

A moderation analysis was conducted to examine the role of socioeconomic status indicators (i.e., income, education, employment status) and ethnicity (i.e., Mexican participants, White participants) on work-related IPV (Figure 1). The moderation analysis was conducted primarily through the use of multiple regression analyses. All continuous variables were mean centered and interaction terms (e.g., income*ethnicity) were created to test for moderation. 


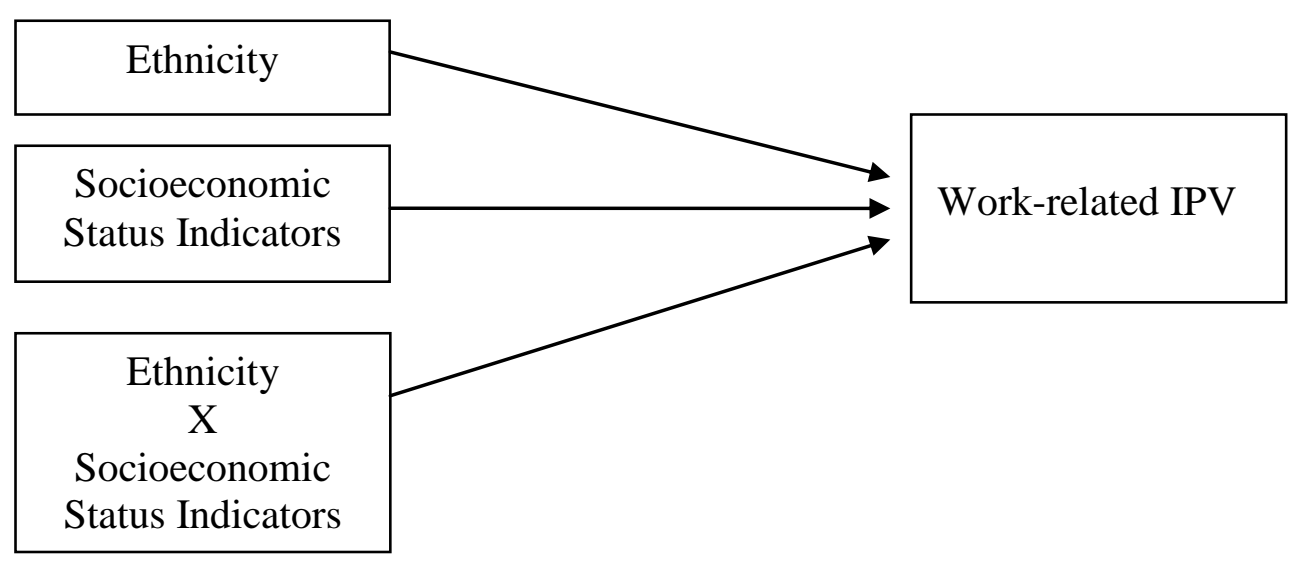

Figure 1. The Moderating Role of Socioeconomic Status Indicators between Ethnicity and Work-related IPV.

A sequential multiple regression analysis was conducted to examine the role of income on work-related IPV and ethnicity. In the first step, two predictors were included: Income and ethnicity (represented by two dummy variables, 0 for Mexican participants, 1 for White participants). This model was statistically significant, $F(2$, $153)=4.545, p=.012$ and accounted for $5.60 \%$ of the variance. Only ethnicity had a significant unique effect $(p<0.01)$, with the ethnicity group change (indicating a group shift from Mexicans to Whites) with overall work-related IPV. The second and final step consisted of adding the interaction term, income and ethnicity. The addition of the interaction term did not significantly increase the model $R^{2}, F(1,152)=1.777, p$ $=0.184$. The results of the analyses suggest that the relationship between ethnicity and work-related IPV did not change as a function of income. 
A sequential multiple regression analysis was conducted to examine the role of education and ethnicity on work-related IPV. In the first step, two predictors were added: education and ethnicity (represented by two dummy variables, 0 for Mexican participants, 1 for White participants). This model was statistically significant, $F(2$, $148)=5.073, p=0.007$ and accounted for $6.40 \%$ of the variance. Neither predictor had a significant unique effect in the model. The second and final step consisted of adding the interaction term, education and ethnicity. The addition of the interaction term did not significantly increase the model $R^{2}, F(1,147)=0.880, p=0.350$. The results of the analyses suggest that the relationship between ethnicity and work-related IPV did not change as a function of education.

Lastly, a sequential multiple regression analysis was conducted to examine the role of employment status and ethnicity on work-related IPV. In the first step, two predictors were added: employment status (represented by two dummy variables, 0 not employed, 1 employed) and ethnicity (represented by two dummy variables, 0 for Mexican participants, 1 for White participants). This model was statistically significant, $F(2,153)=4.244, p=0.016$ and accounted for $5.30 \%$ of the variance in work-related IPV. Only ethnicity had a significant $(p<0.05)$ unique effect in the model. The second and final step consisted of adding the interaction term, employment status and ethnicity. The addition of the interaction term significantly increased the model $R^{2}, F(1,152)=8.651, p=0.004$. The overall model was significant, $F(3,152)=5.854, p=0.001$ and accounted for $10.4 \%$ of the variance. The 
analyses suggest that the relationship between ethnicity and work-related IPV changed as a function of employment status. Specifically, it appears that being unemployed for Mexican participants are associated more with work-related IPV than it was for White participants. The interaction is illustrated in Figure 2. Overall, the analyses indicated partial support of the hypotheses in that only employment status was a significant moderator.

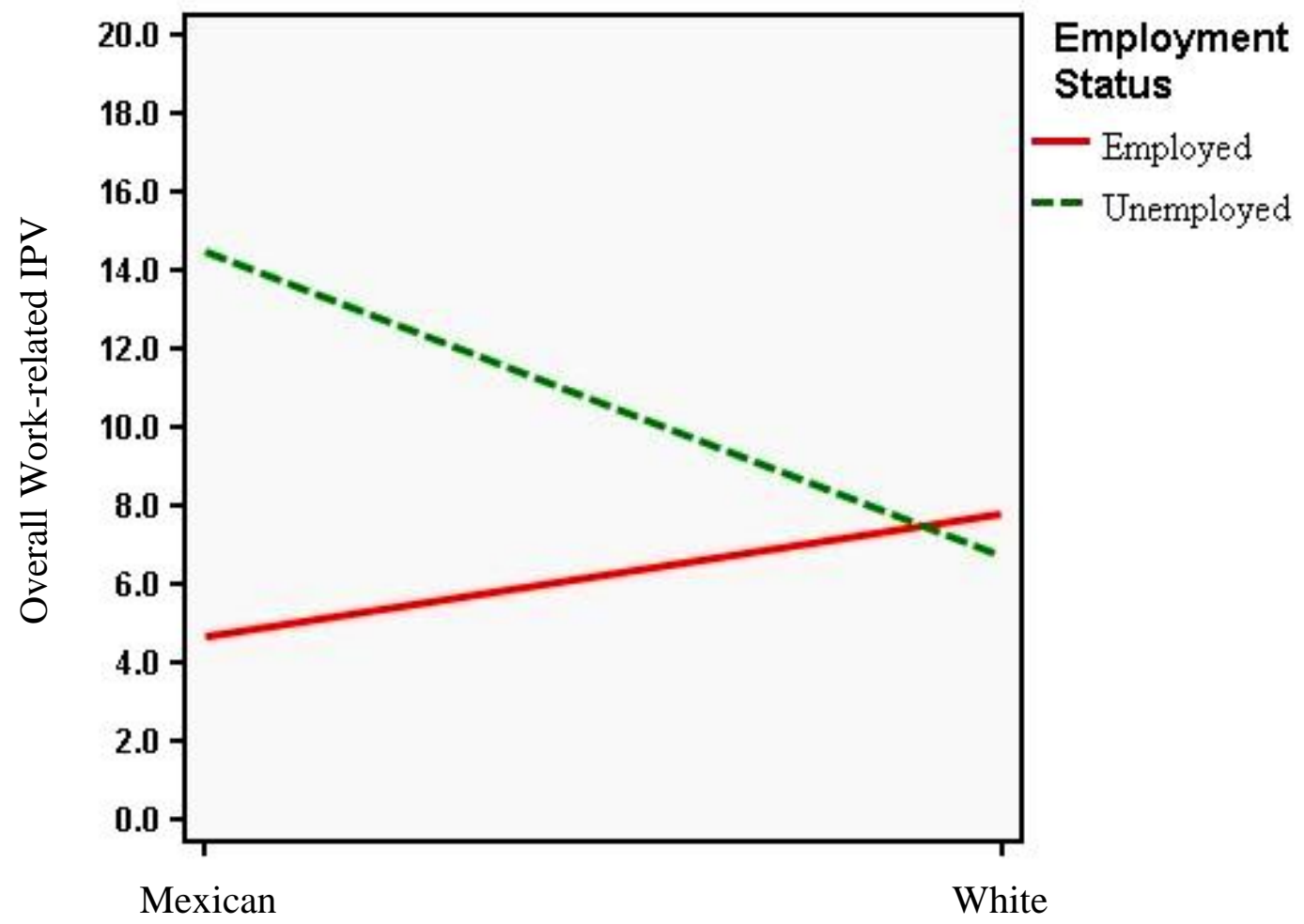

Ethnicity

Figure 2. Employment status moderates the relationship between ethnicity and workrelated intimate partner violence. 
Research Question 3. What are the relationships between four single item indicators of acculturation and a language preference acculturation scale?

A multiple regression analysis was conducted to examine the relationship between proxy indicators of acculturation and acculturation as measured by languagerelated items from the Short Acculturation Scale for Hispanics (SASH). A mean composite variable was created using all the SASH items. The proxy indicators of acculturation were country of birth (e.g., Mexico, U.S.), language of survey (e.g., Spanish, English), age-of-arrival, and number of years in the U.S. Table 10 summarizes the descriptive statistics and analysis results. As a reminder, all analyses from this point forward include only Mexican participants.

The four predictor model was significant and able to account for $56.0 \%$ of the variance in acculturation, $F(4,90)=28.61, p<.001$. As hypothesized, the four acculturation proxy indicators were significantly correlated with the criterion. That is, country of birth (coded as $0=$ Mexico and $1=$ U.S.) is positively and significantly correlated with acculturation, indicating that participants who were born in the U.S. reported higher acculturation scores. The language of the survey was also positively and significantly correlated with acculturation, indicating that those who chose to and completed an English language version of the survey reported higher acculturation scores. In addition, age-of-arrival was negatively and significantly correlated with acculturation, indicating that those participants that arrived in the U.S. when they were younger reported higher acculturation scores. Lastly, the number of years in the U.S. 
was positively and significantly correlated with acculturation, indicating that participants that reported a greater number of years in the U.S. tended to report higher acculturation scores.

While each of the predictor variables had a significant $(p<0.001)$ correlation with acculturation, only country of birth and language of survey had significant $(p<$ 0.001) partial effects in the full model. Specifically, country of birth had a significant positive regression weight, indicating that Mexican participants born in the U.S. were expected to have higher acculturation scores, after controlling for the other variables in the model. Language of survey had a significant positive weight, indicating that after accounting for other variables in the model, participants that completed the English language version of the survey were expected to have higher acculturation scores. Both years in the U.S. and age-of-arrival had little to offer in the context of the other variables and did not contribute to the model.

The analysis indicated that both country of birth and language of survey are more important predictors of acculturation as measured by SASH than the other two proxy indicators. Therefore, country of birth and language of survey were only analyzed in subsequent analyses. 
Work-related IPV 99

Table 10

Multiple Regression for Acculturation Proxy Indicators on Acculturation $(N=100)$

\begin{tabular}{|c|c|c|c|c|c|}
\hline \multirow[b]{2}{*}{ Variable } & \multirow[b]{2}{*}{ Mean } & \multirow[b]{2}{*}{ SD } & \multirow[b]{2}{*}{$\begin{array}{l}\text { Correlation } \\
\text { with SASH }\end{array}$} & \multicolumn{2}{|c|}{$\begin{array}{c}\text { Multiple Regression } \\
\text { Weights }\end{array}$} \\
\hline & & & & $\mathrm{b}$ & $\beta$ \\
\hline Age of arrival & 17.82 & 8.10 & $-0.57 *$ & -0.01 & -0.11 \\
\hline Years in the U.S. & 12.88 & 7.30 & $0.54 *$ & 0.02 & 0.16 \\
\hline Country of birth ${ }^{\mathrm{a}}$ & 0.10 & 0.31 & $0.65^{*}$ & $0.86^{*}$ & 0.34 \\
\hline Language of survey ${ }^{b}$ & 0.10 & 0.30 & $0.63 *$ & $0.79 *$ & 0.31 \\
\hline
\end{tabular}

Note. Born in Mexico, $\mathrm{n}=87$. Born in U.S., $\mathrm{n}=10$. Missing country of birth data, $\mathrm{n}=$ 3. Spanish survey, $\mathrm{n}=90$. English survey, $\mathrm{n}=10$. *Significant at the 0.001 level (2tailed). SASH=Short Acculturation Scale for Hispanics. a. Coded as $0=$ Born in Mexico and $1=$ Born in the U.S.A. b. Coded as $0=$ Spanish and $1=$ English. c. Count.

Research Question 4. What is the role of acculturation and work-related IPV among Latinos enrolled in batterer intervention programs?

In order to determine the relationship between acculturation and work-related IPV, a correlation analysis was conducted. As hypothesized, the analyses indicated a positive and significant relationship between acculturation and overall work-related IPV, $r(98)=0.46, p<.001$ with $20.8 \%$ of the variance for acculturation being associated with the variance in overall work-related IPV. In addition, acculturation as measured by proxy measure country of birth was positively and significantly related to overall work-related IPV, $r(95)=0.50, p<.001$, with $20.79 \%$ of the variance for acculturation being associated with the variance in overall work-related IPV. 
Acculturation as measured by language of survey was positively and significantly related to overall work-related IPV, $r(98)=0.43, p<.001$, with $18.23 \%$ of the variance for acculturation being associated with the variance in overall work-related IPV. Overall, the analyses indicate that participants who reported higher acculturation scores also tended to report higher overall work-related IPV scores.

4b. What is the relationship between socioeconomic status indicators (e.g., income, education, employment status) and acculturation?

A multiple regression analysis was conducted to examine the relationship between socioeconomic status indicators and acculturation. The socioeconomic status indicators were income, education (number of years in school), and employment status (coded as $0=$ not employed and $1=$ employed). Table 11 summarizes the descriptive statistics and analysis results. 
Work-related IPV 101

Table 11

Multiple Regression for Socioeconomic Status Indicators on Acculturation $(N=100)$

Multiple Regression

Weights

\begin{tabular}{lccccc} 
Variable & Mean & SD & $\begin{array}{c}\text { Correlation } \\
\text { with SASH }\end{array}$ & $\mathrm{b}$ & $\beta$ \\
\hline Income & 4.71 & 1.58 & $0.21^{*}$ & 0.05 & 0.11 \\
Education & 8.79 & 3.18 & $0.43^{* *}$ & $0.10^{* *}$ & 0.39 \\
Employment Status $^{\mathrm{a}}$ & 0.96 & 0.20 & $-0.27^{*}$ & $-0.96^{*}$ & -0.24 \\
\hline
\end{tabular}

Note. Employed, $\mathrm{n}=95$. Not employed, $\mathrm{n}=4$. Missing, $\mathrm{n}=1$. *Correlation is significant at the 0.05 level (2-tailed). $* *$ Correlation is significant at the 0.001 level (2-tailed). SASH=Short Acculturation Scale for Hispanics. a. Coded as $0=$ not employed and $1=$ employed. b. Count.

The three predictor model was significant and able to account for $25.30 \%$ of the variance in acculturation, $F(3,94)=10.61, \mathrm{p}<.001$. As hypothesized, two of three socioeconomic status indicators were significantly correlated with the criterion. That is, income is positively and significantly correlated with acculturation, indicating that participants that reported higher incomes tended to report higher acculturation scores. Education was also positively and significantly correlated with acculturation, indicating that those who more education reported higher acculturation scores. Lastly, although the correlation between employment status and acculturation was significant, it was not in the direction hypothesized. The analyses indicated that participants who were not employed tended to report higher acculturation scores. 
While each of the predictor variables had a significant $(p<0.05)$ correlation with acculturation, only education and employment status had significant $(p<0.05)$ partial effects in the full model. Specifically, education had a significant positive regression weight, indicating that participants with a higher number of years of education had higher acculturation scores, after controlling for income and employment status. Employment status had a significant negative weight, indicating that after accounting for education and income, participants who were employed were expected to have lower acculturation scores. Upon closer inspection of predictors, there were only four participants who reported being unemployed. Because of this sample size difference in participants that were employed (96\%) compared to those that were not employed (4\%), employment status will not be used in the subsequent analyses because it will not provide a meaningful interpretation.

A correlation analysis was conducted to examine the relationship between socioeconomic status indicators and acculturation as measured by the proxy variables country of birth and language of survey. The socioeconomic status indicators were income and education (number of years in school). The results are presented in Table 12. Country of birth was positively and significantly correlated with education, indicating that participants who were born in the U.S. tended to report more years of education. In addition, country of birth was positively and significantly correlated with income indicating that participants that were born in the U.S. tended to report higher incomes. Language of survey was positively and significantly correlated with 
education indicating that participants that choose to complete an English language version of the survey tended to report more years of education. However, language of survey was not significantly correlated with income.

Table 12

Correlation Matrix for Socioeconomic Status Indicators and Acculturation Proxy Indicators $(N=100)$

\begin{tabular}{lcccc}
\hline & $\begin{array}{c}\text { Language of } \\
\text { survey }\end{array}$ & $\begin{array}{c}\text { Country of } \\
\text { birth }^{\mathrm{a}}\end{array}$ & Income & Education \\
\hline Language of survey $^{\mathrm{a}}$ & -- & -- & -- & -- \\
Country of birth & & -- & -- & -- \\
Income & $0.55 * *$ & $0.21^{\mathrm{b}}$ & -- & -- \\
Education & 0.15 & $0.31 * *$ & $0.20^{*}$ & -- \\
\hline
\end{tabular}

Note. ${ }^{* *}$ Correlation is significant at the 0.01 level (2-tailed). ${ }^{*}$ Correlation is significant at the 0.05 level (2-tailed). a. Coded as $0=$ Spanish and $1=$ English. $b$. Coded as $0=$ Born in Mexico and $1=$ Born in the U.S.A.

4c. Do socioeconomic status indicators (e.g., income, education) moderate the relationship between acculturation and work-related IPV.

A moderation analysis was conducted to examine the role of socioeconomic status indicators on acculturation and work-related IPV (Figure 3). The moderation analysis was conducted primarily through the use of multiple regression analyses. All continuous variables were centered and interaction terms (e.g., acculturation*income) were created to test for moderation. 


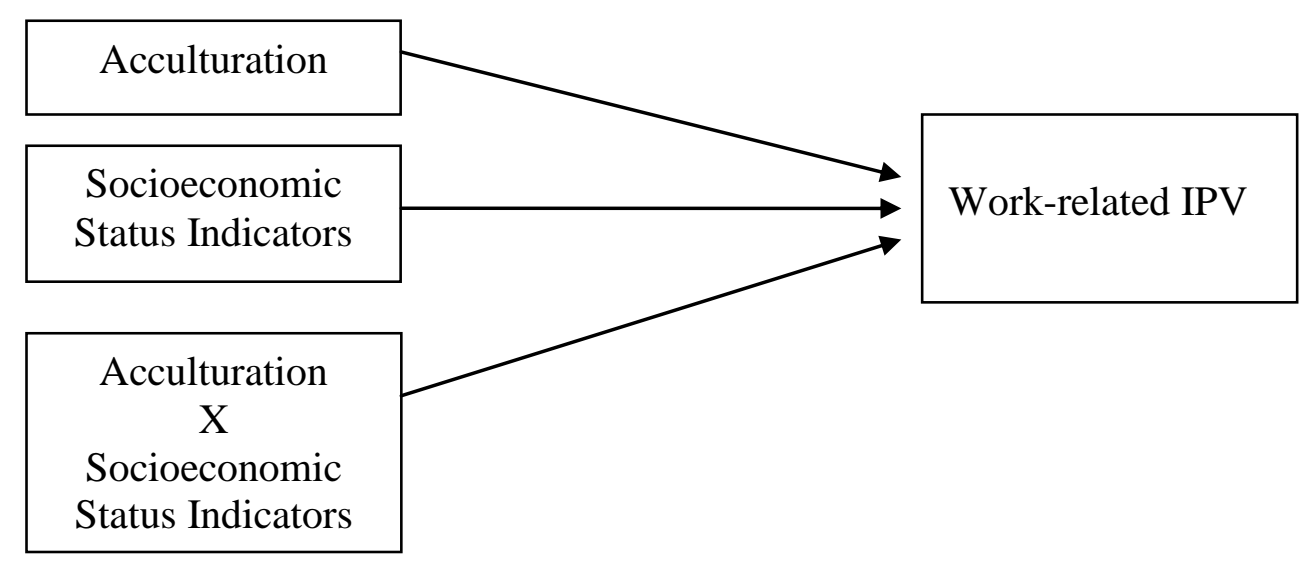

Figure 3. The Moderating Role of Socioeconomic Status Indicators between Acculturation and Work-related IPV.

A sequential multiple regression analysis was conducted to examine the role of income on work-related IPV and acculturation. In the first step, two predictors were added: Income and acculturation (SASH). This model was statistically significant, $F(2,97)=13.65, p<.001$ and accounted for $22.0 \%$ of the variance. Only acculturation had a significant unique effect $(p<0.001)$. The second and final step consisted of adding the interaction term, income and acculturation. The addition of the interaction term did not significantly increase the model $R^{2}, F(1,96)=0.001, p=0.97$. The analyses suggest that the relationship between acculturation and work-related IPV did not change as a function of income.

An additional sequential multiple regression analysis was conducted to examine acculturation as measured by the proxy variable language of survey (Spanish 
or English). In the first step, two predictors were added: income and language of survey. This model was statistically significant, $F(2,97)=12.30, p<.001$ and accounted for $20.2 \%$ of the variance. Only language of survey had a significant unique effect $(p<0.001)$. The second and final step consisted of adding the interaction term, income and language of survey. The addition of the interaction term did not significantly increase the model $R^{2}, F(1,96)=3.82, p=0.054$. The analyses suggest that the relationship between acculturation and work-related IPV did not change as a function of income.

An additional sequential multiple regression analysis was conducted to examine acculturation as measured by the proxy variable country of birth (Mexico or U.S.). In the first step, two predictors were added: income and country of birth. This model was statistically significant, $F(2,94)=16.78, p<.001$ and accounted for $26.3 \%$ of the variance. Only country of birth had a significant unique effect $(p<0.001)$. The second and final step consisted of adding the interaction term, income and country of birth. The addition of the interaction term significantly increased the model $\mathrm{R}^{2}, F(1$, $93)=7.47, p=0.008$. The overall model was statistically significant, $F(3,93)=14.45$, $p<.001$ and accounted for $31.8 \%$ of the variance. The analyses suggest that the relationship between acculturation and work-related IPV changes as a function of income. Specifically, higher income was associated with lower rates of work-related IPV for those born in the U.S., while the opposite relationship exists for those born in Mexico. It appears that for participants born in Mexico (lower acculturation), higher 
income is associated with higher rates of work-related IPV than it is for Mexico-born participants with lower income. The interaction is illustrated in Figure 4.

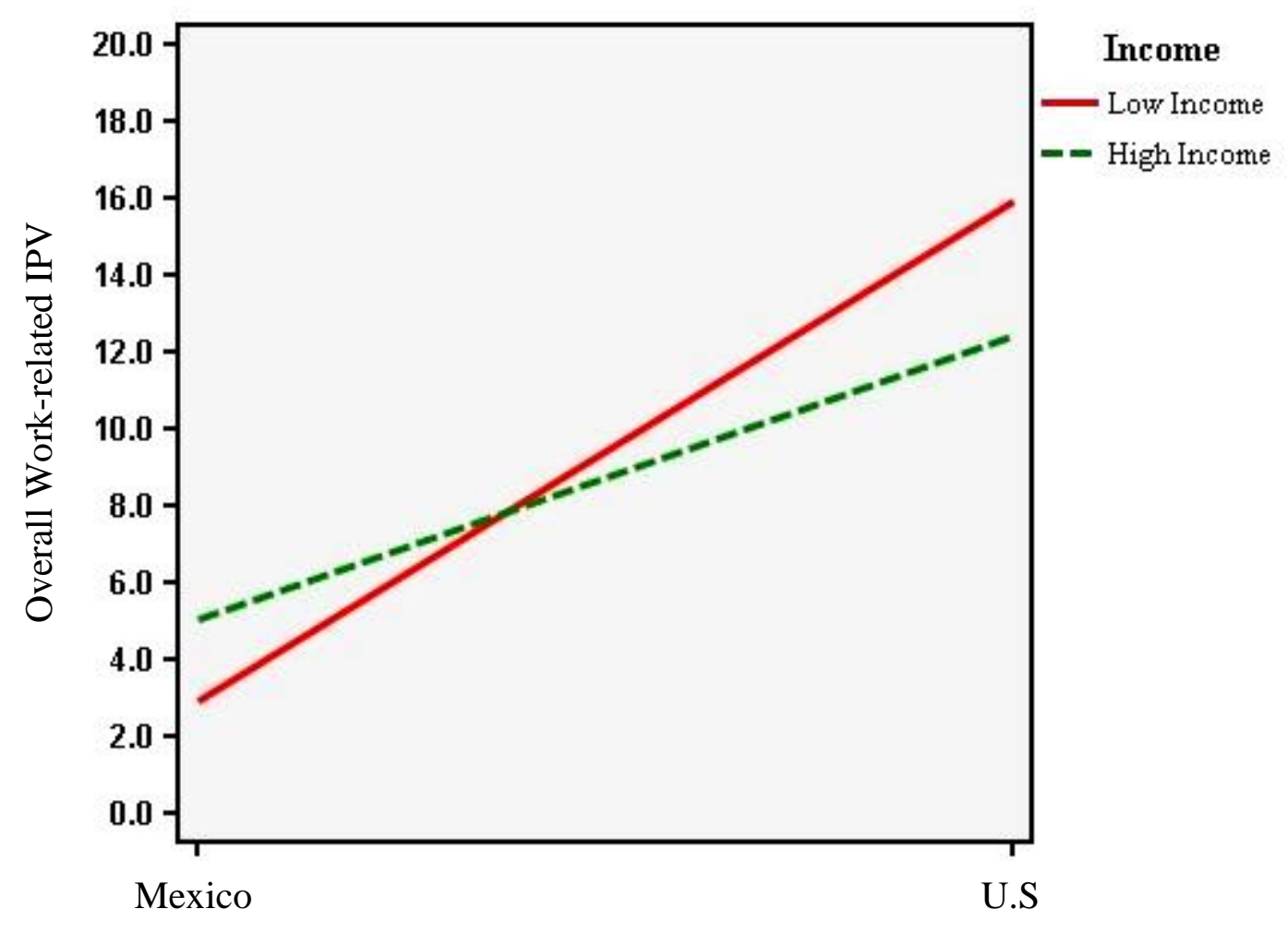

Country of Birth

Figure 4. Income moderates the relationship between acculturation (country of birth) and work-related intimate partner violence.

A sequential multiple regression analysis was conducted to examine the role of education on the relationship between work-related IPV and acculturation. In the first 
step, two predictors were added: education and acculturation (SASH). This model was statistically significant, $F(2,96)=12.65, p<0.001$ and accounted for $20.90 \%$ of the variance. Only acculturation had a significant unique effect $(p<0.001)$. The second and final step consisted of adding the interaction term, education and acculturation. The addition of the interaction term did not significantly increase the model $R^{2}, F(1$, $95)=1.34, p=0.25$. The results suggest that the relationship between acculturation and work-related IPV did not change as a function of education.

An additional sequential multiple regression analysis was conducted to examine the role of education on the relationship between work-related IPV and acculturation as measured by the proxy variable language of survey. In the first step, two predictors were added: education and language of survey. This model was statistically significant, $F(2,96)=11.21, p<.001$ and accounted for $18.90 \%$ of the variance. Only language of survey had a significant unique effect $(p<0.001)$. The second and final step consisted of adding the interaction term, education and language of survey. The addition of the interaction term did not significantly increase the model $R^{2}, F(1,95)=0.027, p=0.87$.

An additional sequential multiple regression analysis was conducted to examine the role of education on the relationship between work-related IPV and acculturation as measured by the proxy variable country of birth. In the first step, two predictors were added: education and country of birth. This model was statistically significant, $F(2,93)=15.42, p<.001$ and accounted for $24.90 \%$ of the variance. Only 
country of birth had a significant unique effect $(\mathrm{p}<0.001)$. The second and final step consisted of adding the interaction term, education and country of birth. The addition of the interaction term did not significantly increase the model $R^{2}, F(1,92)=0.315, p$ $=0.58$. The analyses suggest that the relationship between acculturation and workrelated IPV did not change as a function of education. Overall, the hypothesis that socioeconomic status moderated the relationship between acculturation and reported work-related IPV was partially supported.

Research Question 5. What role do levels of acculturation among couples play in work-related IPV?

This research question explored whether the incongruity of acculturation status between individuals in a couple is related to work-related IPV. Country of birth and number of years in the U.S for both the participant and their partner (as reported by the participant) were used as proxy indicators for acculturation. An index for each participant was created by subtracting the partner's score from the participant's score (i.e., participant's number of years in the U.S. - partner's number of years in the U.S.).

A sequential multiple regression analysis was conducted to examine the relationship between incongruity of acculturation status between individuals in a couple and work-related IPV while controlling for socioeconomic status (e.g., education, income). Table 13 summarizes the descriptive statistics and analysis results. 
Work-related IPV 109

Table 13

Multiple Regression for Acculturation Incongruity Score on Work-related Intimate Partner Violence $(N=87)$

\begin{tabular}{|c|c|c|c|c|c|}
\hline \multirow[b]{2}{*}{ Variable } & \multirow[b]{2}{*}{ Mean } & \multirow[b]{2}{*}{$\mathrm{SD}$} & \multirow[b]{2}{*}{$\begin{array}{l}\text { Correlation } \\
\text { with WIPV }\end{array}$} & \multicolumn{2}{|c|}{$\begin{array}{c}\text { Multiple Regression } \\
\text { Weights }\end{array}$} \\
\hline & & & & $\mathrm{b}$ & $\beta$ \\
\hline Income & 4.84 & 1.61 & $0.23 * *$ & 0.50 & 0.15 \\
\hline Education & 8.87 & 3.27 & 0.15 & 0.22 & 0.13 \\
\hline $\begin{array}{l}\text { Number of years } \\
\text { incongruity score }\end{array}$ & -0.08 & 9.74 & $0.18 * *$ & 0.004 & 0.008 \\
\hline $\begin{array}{l}\text { Country of birth } \\
\text { incongruity score }\end{array}$ & -0.19 & 0.50 & $0.30 *$ & $2.95 * *$ & 0.27 \\
\hline
\end{tabular}

In the first step, two predictors were added: income and education. This model was not statistically significant, $F(2,84)=2.98, p=.056$. The second step added the incongruity of number of years score. This did not significantly increase the model $R^{2}$, $F(1,83)=1.87, p=0.175$. The last step of adding the incongruity of country of birth score significantly increased the model $R^{2}, F(1,82)=4.68, p=0.03$. Overall, the four predictor model was significant and accounted for $13.6 \%$ of the variance in workrelated IPV, $F(4,82)=3.23, p=0.02$. All of the predictors had a significant and positive $(p<0.05)$ correlation with work-related IPV, except education (i.e., number of school years). However, only the incongruity of the country of birth score had a significant unique effect in the model $(p<0.05)$. The incongruity of country of birth 
had a positive regression weight, indicating that a relationship exists between the congruity of country of birth between couples and work-related IPV. Specifically the analyses indicate that the participant being born in the U.S. (higher acculturation status) matched with a partner born in Mexico (lower acculturation status) resulted in a predicted increase of 2.95 in work-related IPV compared to when their status was the same (i.e., either both born in the U.S. or both born in Mexico). Also, the analyses indicate that the participant being born in Mexico (lower acculturation status) matched with a partner born in the U.S. (higher acculturation status) resulted in a predicted decrease of 2.95 in work-related IPV compared to when their status was the same (i.e., either both born in the U.S. or both born in Mexico). This relationship held after controlling for socioeconomic status indicators (income and education) and the incongruity of numbers of years in the U.S. score. A summary of the overall study findings are presented in Table 14. 
Work-related IPV 111

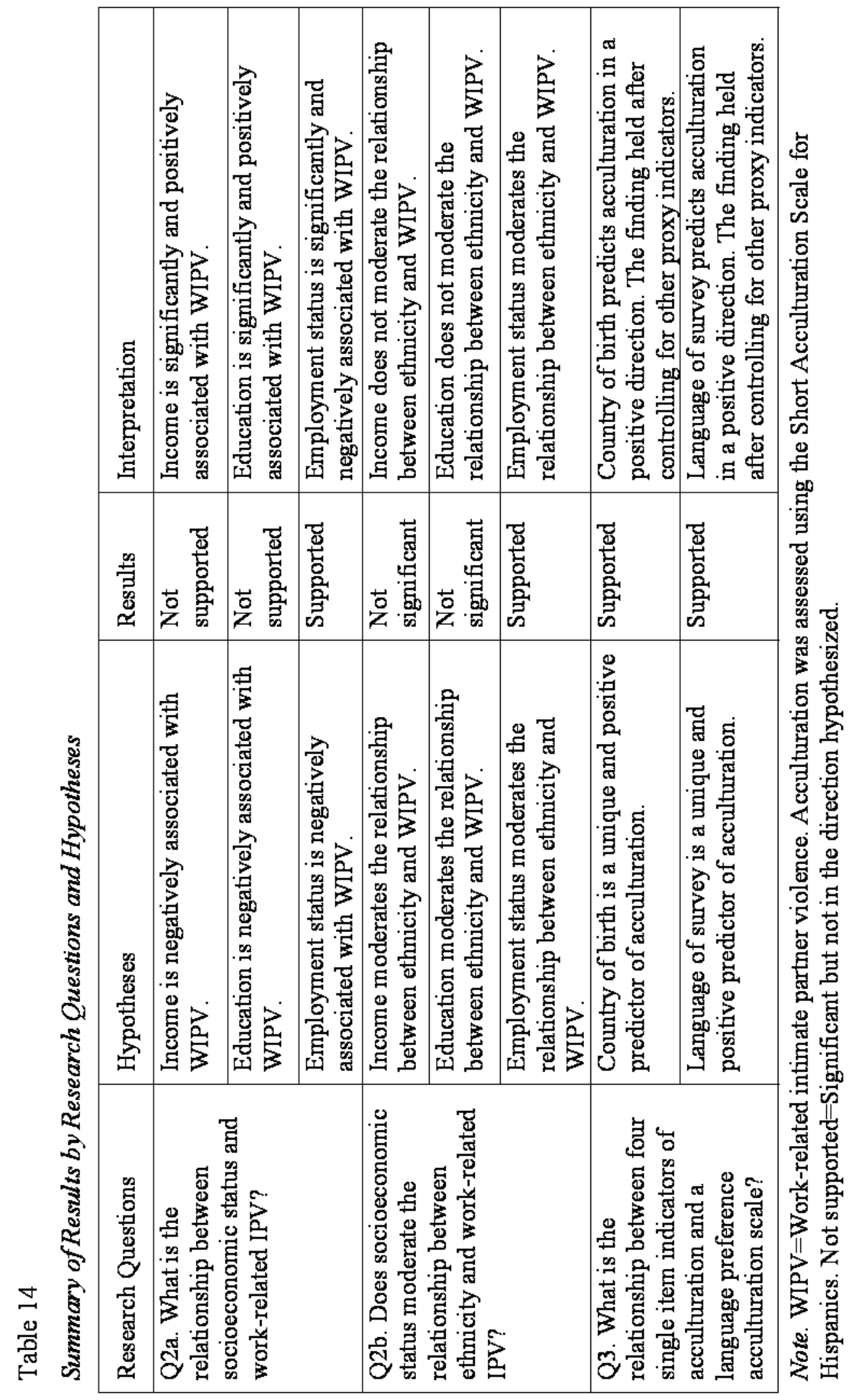


Work-related IPV 112

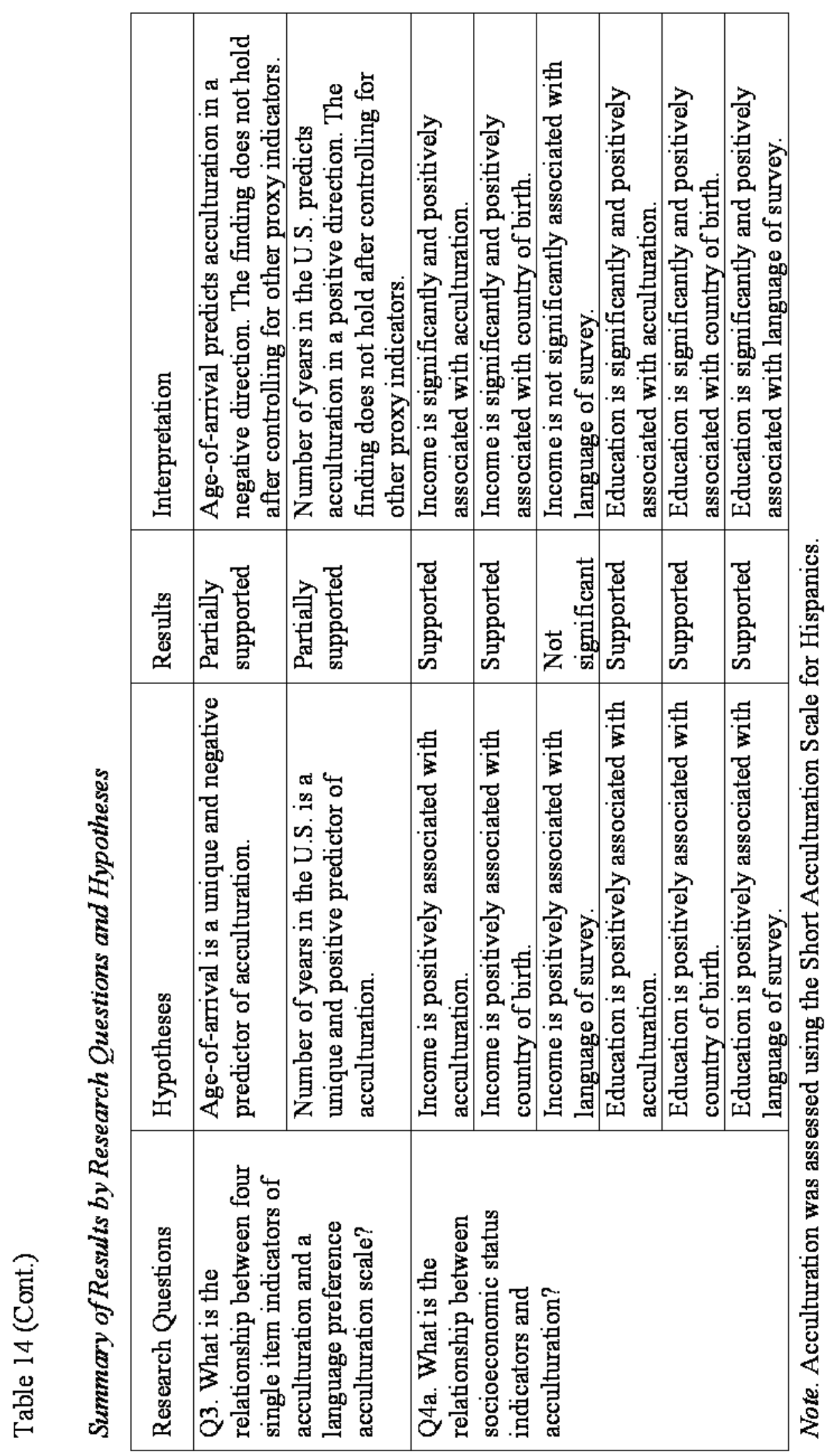


Work-related IPV 113

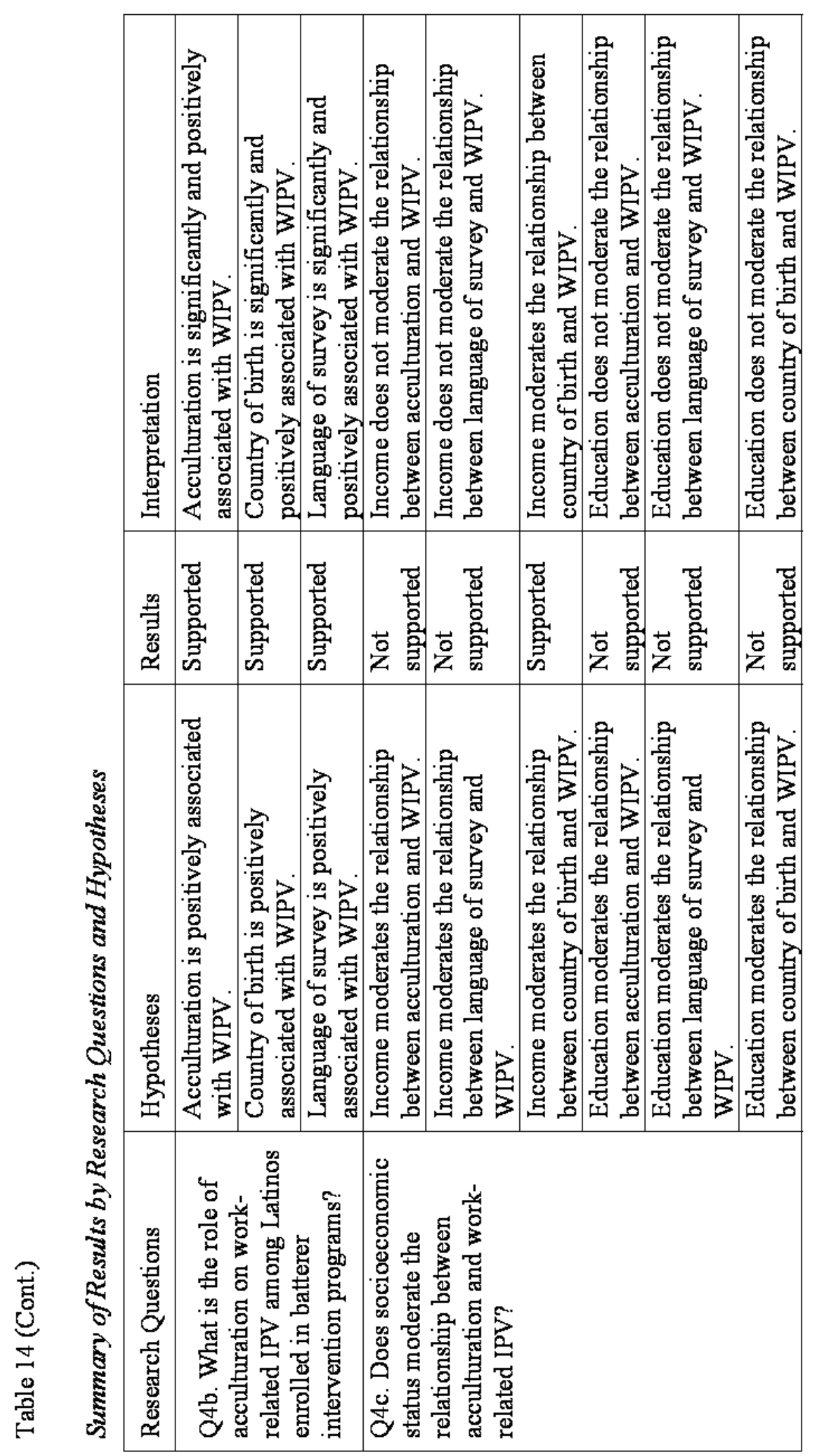




\section{CHAPTER V}

Discussion

The purpose of this study was two-fold. The first goal was to assess the lifetime prevalence of work-related IPV among a sample of Spanish-speaking Latino and English-speaking non-Latino men attending batterer intervention programs. The second goal was to understand and examine the relationship between acculturation, co-related socioeconomic factors, and reported work-related intimate partner violence among Latinos.

\section{Prevalence of Work-related IPV}

One of the important contributions of the study is that it one of the first studies to examine men's reports of work-related IPV. To date, the literature in this area has relied almost exclusively on victim's reports which limit our complete understanding of work-related IPV. Studies are needed that assess men's reported behavior to identify the entire scope of work-related IPV as perpetrated by men. Another important contribution of this study is that we used a sample of men drawn from BIPs. The majority of men $(91.4 \%)$ were court-mandated to attend BIPs as part of their adjudication process. Court-mandated men may be the most appropriate sample in which to examine work-related IPV because they are the group most likely to engage in IPV tactics and behaviors. To date there are very few studies that have examined men's reports of work-related IPV and fewer have sampled known violent men. This 
limitation in reporting has presented an incomplete understanding of the scope of the problem.

The findings in terms of the prevalence of work-related IPV are consistent with prior research among of victims of IPV (Brush 2000; Brush, 2002; Friedman \& Couper, 1987; Swanberg, Logan \& Macke, 2005; Taylor \& Barusch, 2004; Tolman \& Rosen, 2001). We found that $60 \%$ of the entire sample reported yes to one or more questions on the Threatened or Actual Abuse Subscale, indicating that a significant percentage of abusive men engage in work-related tactics that involve threatening behaviors and physical violence at their partner's job. In other words, the majority of the participants in this study reported using or threatening physical violence against their partners at their workplace. Swanberg et al.'s (2005) review of the literature found that abusive men use a variety of specific job interference tactics. In our study, we found that $66 \%$ reported yes to one or more questions on the Interference Subscale indicating that a sizable majority of participants interfered with their partner's ability to get to their work, do their work, and to keep their job. Furthermore, over half of the participants reported tactics that involved interfering with their partner's ability to get to work. Over one-third of the participants reported making their partner late or miss work. Participants reported tactics that interfered with their partner's ability to get to work which included causing their partner to be emotionally traumatized (46\%), injuring their partner (42\%), and interfering with their partner's sleep (35\%). These reports indicate an alarmingly high rate of work-related IPV committed against 
Work-related IPV 116

women. Furthermore, these statistics confirm the upper ranges of previous estimates across studies (36\% to $75 \%$ ) of employed victims of IPV and their harassment by abusive partners while at work (Shepard \& Pence, 1988; Swanberg, Logan, \& Macke, 2005; Taylor \& Barusch, 2004).

Consistent with research by Riger, Ahrens, and Blickenstaff (2000) and Rothman and Perry (2004) among abusive men, tactics related to communication and monitoring were reported in this study. We found that $47 \%$ of the sample reported yes to one or more questions on the Monitor Subscale indicating that a significant number of participants engaged in tactics that involved the surveillance and monitoring of their partners while they were at work (e.g., appearing at partner's work site, interfering with partner's productivity, making frequent telephone calls to partner). The prevalence of these types of tactics, also known as on-the-job harassment, has been estimated between 8\% and 75\% (Brush, 2000; Brush 2002; Friedman \& Couper, 1987; Stanley, 1992; Swanberg et al., 2005; Taylor \& Barusch, 2004; Tolman \& Rosen, 2001). The large range is partly explained by the different samples used in these studies which were primarily drawn from shelters and welfare-to-work programs. Research suggests that victims of IPV from women's shelters and welfare programs may not be representative of employed victims because they typically have experienced more severe forms of IPV which have resulted in their need to flee and/or be underemployed (Rothman et al., 2007). In the current study we found that over half of the participants reported arguing with their partners on the phone while the partner 
was at work. Specifically, these rates are consistent with research among female victims of IPV attending battered women support groups (57\%: Shepard \& Pence, 1988) and among a sample of employed woman who filed domestic violence orders (57\%; Swanberg, Logan \& Macke, 2005). The findings of this study indicate that participants engaged in various tactics that involved the surveillance and monitoring of their partners while they were at work. Overall, the high percentages of work-related IPV reported in this study would result in major consequences for the victimized employee at both the individual (e.g., work performance) and organizational levels (e.g., employee turnover).

One interesting finding suggests that men do not report engaging in severe forms of work-related IPV that involved the use of weapons (both items from the Threatened or Actual Abuse Subscale). That is, none of the participants reported hurting their partners with weapons (e.g., knife, gun) at her place of employment or using their employer's property to hurt their partner. Similarly, only one participant reported threatening to hurt their partner with a weapon at her job. However, there are a few possible alternative explanations for this finding. It is possible that the participants may have under-reported their use of weapons for a few reasons. They may have underreported because the use of serious weapons such as a gun or knife against a women contrast greatly with societal expectations. Under these conditions, participants may have been influenced to not disclose their use of weapons against their partners. It's also possible that they wanted to minimize their use of weapons 
because of the serious consequences (e.g., incarceration) associated with using such weapons. Another possibility is related to the construction of the questions. The additional examples of weapons embedded in each question (e.g., gun, knife) may have narrowed participants' understanding of the questions. Participants may have only considered the weapons that were indicated and may not have thought of using other objects as weapons (e.g., chair), thus limiting their reports. While these alternative explanations are possible, the reports of other severe forms of work-related IPV (i.e., threatening or actual IPV behaviors) that did not involve weapons ranged from $6 \%$ to $46 \%$ indicating that the participants in this study reported a range of tactics that specifically involved physically hurting or threatening to hurt their partners at the partner's work site. For example, $46 \%$ of the participants reported interfering (e.g., making them late or miss work) with their partners' employment by emotionally traumatizing them, $42 \%$ reported interfering with their partners' employment by injuring them, $17 \%$ reported that they threatened to physically hurt their partners at their partners' jobs, and 7\% reported physically hurting their partners at their partners' jobs. Lastly, it's important to note that the particular finding regarding the use of weapons is difficult to compare to other studies because many collapse items regarding the use of weapons into broad categories of "physical violence" or "physical assault" which may include items that range from slapping a partner to using a gun to threaten a partner (Coker, Smith, McKeown, \& King, 2000; Lown \& Vega, 2001; Tjaden \& Thoennes, 2000). 
Work-related IPV 119

\section{Ethnicity and Work-related IPV}

The relationship between ethnicity and IPV has been identified by researchers as an important issue to understand given that data suggest that IPV rates differ between ethnic groups (Caetano, Ramisetty-Mikler, Caetano Vaeth, \& Harris, 2007; Caetano, Schafer, \& Cunradi, 2001). In this study, the findings indicated that White participants reported statistically significantly higher rates of work-related IPV than Mexican participants. Since little empirical data exist on men's reports of work-related IPV, rates are largely unknown. These findings should be interpreted within the context of the literature to date that has examined the relationship between ethnic groups and the prevalence of IPV in general. However, the literature in this regard has produced mixed findings. Many reports of IPV in the general population suggest that Latinos have higher rates of IPV than non-Latino Whites (Caetano, Ramisetty-Mikler, Caetano Vaeth, \& Harris, 2007; Caetano, Schafer, \& Cunradi, 2001) and some reports have shown similar or lower rates compared to other groups from the same communities (Denham et al., 2007; Kantor, 1997; Tjaden, \& Thoennes, 2000). Comparisons between studies are limited because of various methodological issues in terms of study design, sampling frame, type and source of data, and the heterogeneity of Latinos (Klevens, 2007; Perilla, 1999).

There are several interpretations of the current study's finding that differences in reported work-related IPV differed by ethnic groups. Specifically, that White participants reported more work-related IPV than Mexican participants. It is possible 
that White participants may have more access to work-related resources and autonomy at work than Mexican participants. In other words, White participants may have reported more work-related IPV because they had more access to resources such as phones, vehicles, and email, which are used to commit various forms of work-related IPV. Also, because of the types of jobs they held, they may have more autonomy to carry out work-related IPV while at work than Mexican participants. Follow-up analyses were conducted and findings support the interpretation that groups significantly differed in terms of their access to work resources. White participants indeed reported higher percentages of access to email, phones, vehicles, and equipment and tools at their work than Mexican participants.

Another possible interpretation of the finding that rates of work-related IPV differed between groups is that Mexican participants underreported their rates of work-related IPV. One possible explanation for this is that their reports of IPV were influenced by the length of time in the BIP. It's possible that participants who have attended less sessions in the BIP may minimize their IPV and be more in denial about their abusive behavior than participants who have attended a greater number of sessions. It's also possible that the type of BIP that the participants were enrolled in may have influenced the disclosure of work-related IPV. Gondolf and William's (2001) review of the literature found that culturally sensitive BIP counseling produces more positive changes (e.g., less denial, accountability) in batterers than does counseling that does not have any cultural component for ethnic minority men. In this 
Work-related IPV 121

study, we drew from 10 different BIPs. Some of the programs could be categorized as conventional and others as culturally-sensitive. While we did not specifically assess program characteristics in this study, we know that the Latino participants were enrolled in both types of programs. That is, they were enrolled in English-language groups and Spanish-language groups in predominantly English-language programs (i.e., conventional). In addition, some of the participants were enrolled in Spanishlanguage culturally-specific programs (i.e., culturally sensitive). Perhaps, the type of BIP for Mexican participants influenced disclosure of work-related IPV behaviors and tactics. It's possible that Mexican participants in conventional programs underreported work-related IPV more so than participants in culturally sensitive programs.

\section{Socioeconomic Status, Ethnicity and Work-related IPV}

Researchers have sought to explain differences in ethnic groups' rates of IPV by examining the economic factors. Indeed, some research has shown that differences in IPV rates between ethnic groups tend to disappear once socioeconomic status indicators are considered (Caetano, Cunradi, Clark, \& Schafer, 2000; Coker, Smith, McKeown, \& King, 2000; Kantor, Jasinski, \& Aldorondo, 1994). In this study, various socioeconomic status indicators were considered in the relationship between the participants and work-related IPV. Based on the IPV literature, income, education, and employment status were all hypothesized to be negatively related to work-related IPV. However, the hypotheses regarding income and education were not supported. The findings indicated that income and education were significantly and positively 
related to work-related IPV. On the other hand, the hypothesis regarding employment status was supported. That is, the findings indicated that being unemployed was associated with higher reports of work-related IPV than being employed. This finding is consistent with the existing literature on IPV and socioeconomic status, which suggests that an inverse relationship exists between low socioeconomic status and increased risk of IPV (Cunradi, Caetano, \& Shaefer, 2002). The findings of both income and education being positively correlated with work-related IPV are more difficult to interpret. While it's possible that the relationship of these socioeconomic status indicators might play out differently within the specific context of work-related IPV as compared to general IPV, the strength of the relationships between each of the socioeconomic status indicators and work-related IPV were generally small (i.e., ranging from 0.17 to .22; Cohen, 1988).

Given the important relationship that exists between socioeconomic status and IPV in general, this study assessed individual socioeconomic status indicators as moderator variables. It was expected that these socioeconomic status indicators would alter the relationship between ethnicity and reported work-related IPV. The analyses indicate partial support of the hypotheses regarding the moderating role of socioeconomic status indicators (e.g., income, education, employment status) between ethnic groups (i.e., Mexican and White participants) and work-related IPV. Specifically, it was predicted that for both groups, the relationship between ethnicity and work-related IPV is negative, such that reported work-related IPV decreases as 
socioeconomic status indicators increases. The socioeconomic status indicators income and education were not unique predictors of work-related IPV and they did not moderate the relationship between ethnicity and work-related IPV. It's possible that the reason that income and education did not have any additive effects has to do with the characteristics of the sample. In this sample, income and educational levels were low and it is possible that other variables are attenuating the influence of income and education on work-related IPV. Employment status significantly moderated the relationship between ethnicity and work-related IPV. Specifically, the relationship between ethnicity and work-related IPV changed as a function of employment status. For Mexican participants, unemployment status is associated with higher rates of work-related IPV compared to their employed counterparts. Work-related IPV for White participants did not significantly differ based on employment status. The moderating role of employment status suggests that being unemployed for Mexican participants was associated with higher rates of work-related IPV than it was for White participants. Economic distress (e.g., unemployment, stress from economic instability) may trigger men's use of violence against women in intimate relationships (Fagen \& Browne, 1994; MacMillan \& Gartner, 1999). Straus, Gelles, \& Steinmetz (1980) indicate that factors such as low income, unemployment, and part-time employment are associated with an increase of IPV within couples. Findings from a study using neighborhood poverty as predictor of IPV among Whites, Blacks, and Latinos indicated that in the Latino groups, male unemployment doubled a partner's risk of 
being battered (Cunradi, Caetano, Clark, \& Schafer, 2000). Overall, further research is needed to understand the relationship between socioeconomic status indicators and work-related IPV.

\section{Acculturation and work-related IPV}

The acculturation process for Latinos has received attention as a contributing factor to IPV. However, limitations with the measurement of the complex cultural phenomenon have led to contradictory findings regarding its relationship with IPV. This study examined the relationship between a language-based acculturation scale and four proxy indicators in an effort to utilize all available and appropriate measures of the construct. It was hypothesized that country of birth (U.S., Mexico) would be associated with higher acculturation, language of survey (English language, Spanish language) would be associated with higher acculturation, age-of-arrival (younger age) would be associated with higher acculturation, and number of years in the U.S. (greater number of years in the U.S.) would be is associated with higher acculturation. Consistent with the hypotheses, we found significant relationships in the direction proposed for all the proxy indicators of acculturation. It was also hypothesized that all the proxy indicators would be significant unique predictors of acculturation. However, within a multivariate model, only country of birth and language of survey were unique predictors of acculturation. Overall, these findings are consistent with the literature (Marín, Sabogal, Marín, Otero-Sabogal, \& Perez-Stable, 1987; Jasinski, 2001; Kantor et al., 1994; Sorenson \& Telles, 1991). 
The relationship between socioeconomic status indicators (i.e., income, education) and acculturation as measured by a language preference scale and proxy variables country of birth and language of survey was examined. It was hypothesized that socioeconomic status indicators would be positively related to acculturation. Consistent with the hypotheses, significant and positive relationships were found for five out of the six relationships, indicating generally that higher socioeconomic status is associated with higher acculturation. Socioeconomic status and acculturation should strongly be associated with each other because higher levels of income and educational attainment should facilitate the penetration into and interaction with mainstream society (Negy \& Woods, 1992).

This study sought to shed light on the relationship between acculturation and work-related IPV. Analyses were conducted to examine the relationship between acculturation and work-related IPV. Although studies that have examined the relationship between acculturation and IPV among Latinos have produced inconsistent findings (Chun \& Akutsu, 2003; Garcia, Hurwitz, \& Kraus, 2005; Jasinski, 1998; Kasturirangan, Krishnan, \& Riger, 2004; Klevens, 2007), it was hypothesized that acculturation would be positively associated with work-related IPV because highly acculturated individuals may experience greater stress due to greater exposure to conflicting ideals, norms, values, and may be more aware of their limited access to social, educational, and economic opportunities (Balls Organista, Organista, \& Kurasaki, 2003; Firestone, Lambert, \& Vega, 1999). Consistent with the hypothesis, a 
significant positive relationship was found between acculturation and work-related IPV. Furthermore, significant positive relationships were found between proxy indicators of acculturation and work-related IPV. These findings are consistent with literature that suggests that higher levels of acculturation are associated with higher rates of IPV (Caetano, Schafer, Clark, \& Cunradi, 1998; Caetano, Schafer, Clark, Cunradi, \& Raspberry, 2000; Firestone, Lambert, \& Vega, 1999; Ingram, 2007; Jasinski, 2001; Kantor, Jasinski, \& Aldorondo, 1994).

Researchers have suggested various pathways in which higher acculturation among Latinos are associated with increased IPV. Sorensen and Telles (1991) posit that more acculturated Latinos may use violence toward their partners because they are trying to balance the demands placed on them by their culture of origin and the dominant culture to which they are trying to adapt to. For Latinos and other ethnic minority groups, acculturation implies a multitude of changes. Among Latinos, changes in gender roles and expectations, familial obligations, and beliefs regarding the use of partner violence have been linked to increased contact with U.S. mainstream culture (Sabogal, Marín, Otero-Sabogal, Marín, \& Perez-Stable, 1987). These types of changes may produce more conflict among Latino couples, which could then lead to increased risk for IPV (Torres, 1991). For example, researchers have suggested that IPV might occur as a result of changes within traditional gender roles or when they become destabilized (Klevens et al., 2007; Morash, Bui, \& Santiago, 2000). 
Another explanation involves research which examines the stress experienced by ethnic minorities. Some research suggests that perceptions and/or actual experience of discrimination increases with longer periods of time spent in the U.S. for ethnic minorities (Portes \& Rumbaut, 1990). Furthermore, the process of acculturation in it of itself is a crucial factor that contributes to stress and anxiety among immigrant groups (Rivera-Sinclair, 1997). For ethnic minorities, the conflict between cultures and societal inequalities creates stress, which may increase family conflict and risk for violence (Huo, Smith, Tyler, \& Lind, 1996; West, 1997).

A moderation analysis was conducted to examine the role of socioeconomic status indicators in the relationship between acculturation and work-related IPV. Given that low socioeconomic status has been consistently linked to higher rates of IPV among Latinos and normative samples (Cunradi, Caetano, \& Schafer, 2002; Gelles, 1985; Kantor, Jasinski, \& Aldorando, 1994; Pearlman, Zierler, Gjelsvik, Verhoek-Oftedahl, 2003; Straus \& Smith, 1995; Sugihara \& Warner, 2002; Torres, 1991), it was hypothesized that socioeconomic status would moderate the relationship between acculturation and work-related IPV. Contrary to what was hypothesized, socioeconomic status (i.e., income, education) did not moderate the relationship between acculturation and work-related IPV. The pattern of findings generally held in all the analyses using the proxy indicators for acculturation with the exception of one significant interaction between income, country of birth and work-related IPV (and one marginally non-significant interaction between income, language of survey, and 
work-related IPV). Specifically, the significant interaction indicated that for participants born in Mexico (i.e., lower acculturation), higher income was associated with higher rates of work-related IPV than it was for Mexico-born participants with lower income.

Overall, the hypothesis that socioeconomic status would moderate the relationship between acculturation and work-related IPV was not supported. One likely explanation for this finding has to do with the relationship between acculturation and socioeconomic status. In every multivariate model, acculturation as measured by a language preference scale or proxy was a unique significant positive predictor of work-related IPV. Socioeconomic status indicators had very little to offer in the context of acculturation and they did not significantly contribute to the model (with the exception of the interaction between income, country of birth and workrelated IPV). Although the relationships between socioeconomic status indicators and acculturation were mostly significant, the strength of the relationships ranged from small to medium in strength. It's possible that the reason that income and education did not have any additive effects had to do with the characteristics of the sample. That is, in this sample both income and educational levels were low. On average, participants reported 8 years of education and monthly income range of $\$ 1,001$ and $\$ 1,500$ a month which represent a lower socioeconomic status. Additionally, the relationship between each of the socioeconomic status indicators and work-related IPV were generally small which may explain why socioeconomic status in this sample 
did not moderate the relationship between acculturation and IPV. It is possible that in a non-BIP sample of abusive men, the strength of the relationship between socioeconomic status, acculturation, and work-related IPV might be different.

Socioeconomic indicators measured in this study seem to accurately reflect the population of Latinos in Oregon, who are mostly of Mexican origin. In terms of economic status in Oregon, U.S. Census data indicate that the median annual income of Latinos is $\$ 18,331$ which is substantially lower than Whites and African Americans, \$29,839 and \$27,395, respectively (Pew Hispanic Center, 2008). Furthermore, the poverty rate is highest among Latinos and educational attainment levels are low (Pew Hispanic Center, 2008). In a national survey of 2,544 Latinos in the U.S., when compared to their Latino counterparts (i.e., Cubans, Puerto Ricans, Other Latinos), Mexicans had fewer years of formal education, lower household incomes, and experienced the most acculturative distress (Guamaccia, Pincay, Alegria, Shrout, Lewis-Fernandez, \& Canino, 2007).

The consideration of socioeconomic status remains an important issue in the examination of acculturation among Latinos and especially among Mexicans in the U.S. While this study did not support a moderating role of socioeconomic status between acculturation and work-related IPV, the results suggest that socioeconomic status and acculturation are positively related. Negy and Woods (1992) recommend that research involving acculturation needs to assess socioeconomic status in order to clarify the specific influences of the two constructs. They also note that caution should 
Work-related IPV 130

be taken in understanding the direction and strength of the relationship between acculturation and socioeconomic status because it remains unclear whether being more acculturated facilitates interaction with U.S. mainstream which in turn results in better living standards and access to education or whether higher socioeconomic status facilitates becoming more acculturated (Negy \& Woods, 1992).

\section{Acculturation Status among Couples and Work-related IPV}

To further investigate the relationship between acculturation and work-related IPV, an analysis was conducted to examine acculturation discrepancy between members of couples. Some research has suggested that certain acculturation patterns, in particular, mismatched levels (e.g., lower and higher), may increase IPV because of different expectations regarding roles within the couples (Caetano, Schafer, Clark, Cunradi, \& Raspberry, 2000). In this study, acculturation was assessed using two proxy indicators for both the participants and their reports about their partners. The analyses indicate that a relationship exists between the incongruity of acculturation status of couples and work-related IPV. Specifically, country of birth is associated with more work-related IPV when a participant was born in the U.S. (i.e., more acculturated than the partner because partner was born in Mexico) than compared to couples in which partners were matched in terms of country of birth (i.e., both either born in U.S. or born in Mexico). Furthermore, the analyses suggested that being born in the U.S. for the partner was associated with lower work-related IPV when compared to couples in which they were matched in terms of country of birth (i.e., both either 
born in U.S. or born in Mexico). It's possible that more acculturated Latinos may use violence toward their partners because they are experiencing more changes in their gender roles and expectations than their partners. Researchers have theorized that changes or destabilization of traditional gender roles may produce more conflict among Latino couples (Klevens et al., 2007; Morash, Bui, \& Santiago, 2000; Torres, 1991).

While it is plausible that mismatched acculturation status (e.g., lower and higher) between couples may increase IPV because of different expectations regarding roles within the couples or the experience of acculturative stress (Caetano, Schafer, Clark, Cunradi, \& Raspberry, 2000), research in this area has not been extensively conducted. Two studies have generally found that the discordance between couples' acculturation levels were not associated with an increase of IPV among Latinos (Caetano, Ramisetty-Mikler, \& McGrath, 2004; Caetano, Schafer, Clark, Cunradi, \& Raspberry, 2000) and one study found that Latina's employment created several sources of intracouple conflicts by challenging gender role norms and expectations (Gryywacz, Rao, Gentry, Marin, \& Arcury, 2009). Regarding Caetano et al.'s research, there are some important differences that could explain the results of the current study. Caetano et al. assessed acculturation for both partners using a scale while the present study used acculturation proxy variables gathered from only one participant, which may be less valid. Furthermore, Caetano et al. were able to create three different acculturation status groups (i.e., low, medium, high) using criteria 
based on a national household survey for Latinos which allowed for more specific comparisons. Overall, their measurement of acculturation is likely more sensitive to variations in acculturation than the current study's method of using proxy variables, which could explain the relationship that we found. In one qualitative study that examined IPV among Mexican immigrant men and women, women's entrance into paid employment following migration to the U.S. was perceived differently by each group. The differences reported by both groups suggested asymmetry in the rate of acculturation (Gryywacz, Rao, Gentry, Marin, \& Arcury, 2009). More research is needed to elucidate the relationship of acculturation status between couples and workrelated IPV.

\section{Limitations}

Several features of the research design limit the ability to draw conclusions about work-related IPV. First, the data were not collected from a nationally representative sample that would make the findings more generalizable. The sample was drawn from selected BIPs in rural and urban areas throughout the state of Oregon, which is an improvement on a simple convenience sample. However, it is possible that participants from these types of program in Oregon are different in terms of reported work-related IPV than other abusive men enrolled in BIPs in other states. For example, the majority of Latinos in Oregon originate from Mexico and come from rural communities where most adults commonly have very little formal education (McGlade, 2002). Given the relationship between education and employment, it is 
plausible that work-related IPV would differ between other Latino immigrant groups that have different migration contexts in different parts of the U.S. Overall, since this is one of the first studies to examine work-related IPV among men enrolled in BIPs, possible differences in work-related IPV lifetime rates are unknown.

Second, the frequency and mode of work-related IPV may be different among abusive men who have not been adjudicated by the criminal justice system and mandated to attend BIP. In other words, abusive men in BIP may be different than abusive men who have not been detected or arrested for IPV. It is possible that men who have been arrested for perpetrating IPV may use different or more severe forms (e.g., physical abuse) of work-related IPV than men who have not been arrested. On the other hand, perhaps men who have not been arrested engage in more frequent but less severe forms of work-related IPV.

Third, self-report is always a limitation in studies of sensitive topics. There are several corresponding concerns with data collected in this manner (e.g., response bias). For example, participants may have under-reported work-related IPV due to the stigma associated with IPV. In addition, social desirability bias is a concern because it may influence responses when the context contrasts greatly with societal norms (i.e., norms against IPV). However, there were several measures that we undertook to minimize bias and increase the validity of response. The use of a survey was deliberate and is an advantage over other methods of IPV-related data collection. Research by Szinovacz and Egley (1995) found that abusive partners were more likely to deny IPV 
in a face-to-face interview than submitting a survey to an administrator. We spent a considerable amount of resources to develop and pilot test (e.g., bilingual/bicultural team of translators/researchers) a Spanish-language version of the survey. Providing a Spanish language survey to the participants helped build rapport and minimize bias in their reports. In addition, open-ended items were developed so as to provide participants with the opportunity to communicate their perspectives. Lastly, we developed an alternate mode of audio survey administration using an iPod that was more accessible to participants with lower literacy which was also available in the Spanish language.

Fourth, the cross-sectional design of the study limits the interpretation of causal relationships. That is, the observed associations between key study variables cannot be interpreted as causal processes. For example, it is possible that work-related IPV could predict level of acculturation among Latinos. It is possible that a greater number of particular types of tactics such as the use of work resources may be more characteristic of higher acculturated Latinos than less acculturated ones. The use of work resources may be associated with greater autonomy on the job, higher pay, lessstructured tasks, and professional types of jobs. These aspects may be correlated with an employee with a higher level of acculturation (e.g., access to education, adoption of majority group norms and values, etc.).

Due to space limitations on the survey and theoretical differences among the larger research team regarding the conceptualization of acculturation, we were not 
able to include several additional measures of Latino culture (e.g., familism) and acculturation (e.g., multidimensional models). Due to the linear nature of the acculturation measures used in this study, individuals' bicultural identities are ignored and they are lumped into a lower or higher acculturation status. An orthogonal assessment of acculturation would allow researchers to examine the relative contribution of a given cultural identity to a particular outcome, which would increase our understanding of the multidimensional complexities underlying the process of acculturation and biculturalism (Rodriguez, Mira, Paez, \& Myers, 2007).

Finally, some researchers have noted that the variation of prevalence rates of IPV among different ethnic groups across studies can be influenced by various factors such as the specific population being studied (e.g., rural or urban samples) or the time interval for which participants are being asked to report IPV (e.g., past 6 months, lifetime). In this study, we chose to assess lifetime rates primarily because there is currently no information about the prevalence of work-related IPV behaviors. However, it is possible that rates of work-related IPV might be different if we used a different time interval (e.g., 6 months). Also, some or all of the reported work-related may have occurred before migration to the U.S. A lifetime assessment is probably not as valid as measures which have shorter periods of time and may not capture all of the actual variance in work-related IPV that exists in the population, thus limiting the ability to find correlates and predictors of work-related IPV. However, this study found many of the hypothesized relationships which suggest that this limitation was 
Work-related IPV 136

overcome. In addition, it is possible that stronger relationships between the measured variables could exist than were detected with this relative imprecise measure. Future research should ascertain whether there are time interval differences in rates of reported work-related IPV.

\section{Implications}

This study makes important contributions to the literature and has implications for employers. This is the first study to examine work-related IPV using survey data collected from abusive men enrolled in batterer intervention programs. The reports of work-related IPV in this study corroborate previous findings (e.g., types of tactics) and overall estimates are consistent with the higher ranges of previous estimates across studies. The significant rates of work-related IPV found in this study suggest that it is an important problem that needs to be addressed. Research suggests that work-related IPV creates severe consequences for employed victims of IPV and their employers. Increasing the awareness of the impact that IPV causes on both the individuals involved and employers might help reduce the social and economic costs associated with work-related IPV. Employers may raise awareness of this issue by creating policies that address work-related IPV. It has been estimated that over $70 \%$ of workplaces in the U.S. do not have formal programs or policies that address workrelated violence (BLS, 2006).

As the awareness of work-related IPV grows, employers will feel pressure to respond with effective training for employers and employees (Rothman \& Perry, 
2004). Data from this study could be used to develop materials (e.g., policies, pamphlets, posters, community resources) to present to employees by their employers. Employers could be educated on the types of workplace resources that abusive employees use to perpetrate work-related IPV. In general, training materials could be developed to educate employers about IPV. As part of the larger study, Glass, Bloom, Perrin, and Anger (2010) developed a computer-based training intervention for supervisors and managers to respond to work-related IPV. Data from the current study was used on many of the informational slides featured in the training intervention program. Overall, results of the evaluation indicated that the training intervention was highly effective in: a) conveying work-related IPV information, b) changing perceptions, and c) developing an intention to address work-related IPV. Training programs such as this one could be a useful way of training employers about workrelated IPV.

The findings of this study are particularly relevant given the recent climate in Oregon. In 2007, the state of Oregon passed the Senate Bill 946 which outlines employers' responsibilities in providing unpaid leave for employees that are victimized by IPV. While efforts are rightly being made to ameliorate the negative impact of IPV among employed women, by surveying mostly employed men regarding their use of work-related IPV tactics and behaviors, this study highlights the need to address this problem among employed males as an important step in preventing work-related IPV. 
This study makes important contributions to the literature regarding Latinos and IPV. Researchers have noted the need to examine economically diverse samples to further understand and address work-related IPV (Swanberg, Logan, \& Macke, 2005). This study is the first to examine acculturation and other co-related socioeconomic factors as it relates to work-related IPV among Latinos. The level of acculturation and factors such as income, employment, and education are important contextual factors that provide a better understanding of IPV in Latino communities. Specifically, the findings of this study support the limited data that suggests that an important relationship exists between acculturation, IPV, and employment (Gryywacz, Rao, Gentry, Marin, \& Arcury, 2009). This study is an improvement over other studies that have assessed acculturation by using only one indicator. By using various measures to assess acculturation, this study highlights the importance of considering acculturation as a complex construct and may offer a better assessment of the construct. More refined assessments of acculturation are needed to address the various methodological limitations discussed in the literature and they may offer a better understanding of the relationship between acculturation and work-related IPV by teasing out which indicators best correlate with IPV. Despite limitations in the measurement of acculturation in this study (e.g., non-multidimensional measures), these findings contribute to the body of research that has found an association with higher levels of acculturation and IPV among Latinos. 
Developing interventions that target normative settings such as the workplace might be an effective approach in addressing IPV for Latinos. Research among Latina victims of IPV indicates that they are less likely to utilize social services and healthcare systems because of a host of culturally-specific barriers (Lipsky, Caetano, Field, \& Larkin, 2006). Therefore, services and intervention models that reach Latinos in normative settings in which they participate in may be more effective at reducing the incidence and recurrence of IPV. In addition, the findings related to Latinos could be used to inform the development of culturally-specific BIP interventions. These results support prevention strategies that would take into account the cultural context of Latinos. For example, BIP providers could address work-related IPV in their programs and discuss ways in which employment may change or destabilize traditional gender roles and expectations among Latinos. In Latino communities, programs or classes could discuss and teach alternative non-abusive means to reduce conflict among employed couples that are experiencing changes as a result of the acculturation process. 


\section{References}

Adames, S. B., \& Campbell, R. (2005). Immigrant Latinas' conceptualizations of intimate partner violence. Violence Against Women, 11, 1341-1364.

Adams, A. E., Sullivan, C. M., Bybee, D., \& Greeson, M. R. (2008). Development of the scale of economic abuse. Violence Against Women, 14, 563-588.

Archer, J. (2000). Sex differences in aggression between heterosexual partners: A meta-analytic review. Psychological Bulletin. 126, 651-680.

Armstrong, K., Ravenell, K. L., McMurphy, S., \& Putt, M. (2007). Racial/ethnic differences in physician distrust in the United States. American Journal of Public Health, 97, 1283-1289.

Bachman R. (1994). Violence against women: A national crime victimization survey report. Washington, D.C.: Bureau of Justice Statistics, U.S. Dept. of Justice.

Balls Organista, P., Organista, K., \& Kurasaki, K. (2003). The relationship between acculturation and ethnic minority mental health. In K. Chun, P.B. Organista, \& G. Marín (Eds.), Acculturation: Advances in theory, measurement, and applied research (pp. 139-161). Washington, DC: American Psychological Association.

Bauer, H. M., Rodriguez, M. A., \& Perez-Stable, E. J. (2000). Prevalence and determinants of intimate partner abuse among public hospital primary care patients. Journal of General Internal Medicine, 15, 811-817. 
Bauer, H. M., Rodriguez, M. A., Quiroga, S. S., \& Flores-Ortiz, Y. G. (2000). Barriers to health care for abused Latina and Asian immigrant women. Journal of Health Care for the Poor and Underserved, 11(1), 33-44.

Becerra, R. M. (1988). The Mexican-American family. In C. H. Mindel, R. W. Habenstein,\& R. Wright (Eds.), Ethnic families in America, patterns and variations (pp. 153-171). Upper Saddle River, NJ: Prentice Hall.

Berry, J.W. (1997). Immigration, acculturation, and adaptation. Applied Psychology: An International Review, 46, 5-68.

Berry, J. (2003). Conceptual approaches to acculturation. In K. Chun, P. Balls Organista, \& G. Marín (Eds.), Acculturation: Advances in theory, measurement and applied research (pp. 17-37). Washington DC: American Psychological Association.

Berry, J.W., Trimble, J. E., \& Olmedo, E. L. (1986). Assessment of acculturation. In W. Lonner \& J. Berry (Eds.), Field methods in cross-cultural research (pp. 291-324). Thousand Oaks, CA: Sage.

Berry, J. W., Poortinga, Y. H., Segall, M. H., \& Dasen, P. R. (2002). Cross-cultural psychology research and applications. $2^{\text {nd }}$ Edition. Cambridge, UK: Cambridge University Press.

Bhopal, R. (1997). Is research into ethnicity and health racist, unsound, or important science? British Medical Journal, 314, 1751-1756. 
Brislin, R. W. (1986). The wording and translation of research instruments. In W. J. Lohner \& J. W. Berry (Eds.), Field methods in cross-cultural research (pp. 137-164). Beverly Hills: Sage.

Brownwell, P. (1996). Domestic violence in the workplace: An emergent issue. Crisis Intervention, 3, 129-141.

Bui, H. N., \& Morash, M. (1999). Domestic violence in the Vietnamese immigrant community: An exploratory study. Violence Against Women, 5, 769-795.

Bureau of Labor Statistics (BLS), U.S. Department of Labor. (2006, October). Survey of Workplace Violence Prevention, 2005. Retrieved March 21, 2011 from http://www.bls.gov/iif/oshwc/osnr0026.pdf.

Cabassa, L. J. (2003). Measuring acculturation: Where we are and where we need to go. Hispanic Journal of Behavioral Sciences, 25, 127-146.

Campbell, J. C. \& Lewandowski, L.A. (1997). Mental and physical health effects of intimate partner violence on women and children. Anger, Aggression and Violence, 20, 353-373.

Campbell, D. W., Masaki, B. \& Torres, S. (1997). Water on rock: Changing domestic violence perceptions in the African American, Asian American and Latino communities. In E. Klein, J. Campbell, E. Soler \& M. Ghez (Eds.), Ending domestic violence: Changing public perceptions/halting the epidemic. Thousand Oaks, CA: Sage. 
Caetano, R. C., Schafer, J., Clark, C. L., Cunradi, C. B., \& Raspberry, K. (2000). Intimate partner violence, acculturation, and alcohol consumption among Hispanic couples in the United States. Journal of Interpersonal Violence, 15, 30-45.

Caetano, R., Field, C. A., Ramisetty-Mikler, S., \& McGrath, C. (2005). The 5-year course of intimate partner violence among White, Black, and Hispanic couples in the United States. Journal of Interpersonal Violence, 20, 1039-1057.

Caetano, R., Ramisetty-Mikler, S., Caetano Vaeth, P. A., Harris, T. R. (2007). Acculturation stress, drinking, and intimate partner violence among Hispanic couples in the U.S. Journal of Interpersonal Violence, 22, 1431-1447.

Carrillo, R., \& Zarza, M. J. (2008) Fire and firewater: A co-occurring clinical treatment model for domestic violence, substance abuse, and trauma. In R. Carrillo, \& J. Tello (Eds.), Family violence and men of color healing the wounded male spirit (pp. 61-84). Springer Publishing Company, New York.

Champion, J. D. (1996). Woman abuse, assimilation, and self-concept in a rural Mexican American community. Hispanic Journal of Behavioral Sciences, 18, 508-521.

Chun, K. M., \& Akutsu, P. D. (2003). Acculturation among ethnic minority families. In K. M. Chun, P. B. Organista, \& G. Marín (Eds.), Acculturation: Advances in theory, measurement, and applied research, 95-119. Washington DC: American Psychological Association. 
Cohen, J. (1988). Statistical power analysis for the behavioral sciences (2nd edition). Hillsdale, New Jersey: Erlbaum.

Coker, A. L., Smith, P. H., McKeown, R. E., \& King, M. R. (2000). Frequency and correlates of intimate partner violence by type: Physical, sexual, and psychological battering. American Journal of Public Health, 90, 553-559.

Comas-Diaz, L. (1995). Puerto Ricans and sexual child abuse. In L. Fontes (Ed.), Sexual Abuse in Nine North American Cultures: Treatment and Prevention. Thousand Oaks, CA, US: Sage Publications, Inc; Thousand Oaks, CA, US: Sage Publications, Inc.

Cunningham, W. E., Burton, T. M., Hawes-Dawson, J., Kington, R. S., \& Hays, R. D. (1999). Use of relevancy ratings by target respondents to develop healthrelated quality of life measures; an example with African-American elderly. Quality of Life Research, 8, 749-768.

Cunradi, C. B., Caetano, R., Clark, C., \& Schafer, J. (2000). Neighborhood poverty as a predictor of intimate partner violence among white, black, and Hispanic couples in the United States: A multilevel analysis. Annals of Epidemiology 10, 297-308.

Cunradi, C. B., Caetano, R., \& Schafer, J. (2002). Socioeconomic predictors of intimate partner violence among White, Black, and Hispanic couples in the United States. Journal of Family Violence, 17, 377-389. 
Day, J. C. (1996). Population projections of the United States by age, sex, race, and Hispanic origin: 1995 to 2050. U.S. Bureau of the Census, Current Population Reports, P25-1130, U.S. Government Printing Office, Washington, DC.

Denham, A. C., Frasier, P.Y., Hooten, E. G., Belton, L., Newton, W., Gonzalez, P., Begum, M. \& Campbell, M. K. (2007). Intimate partner violence among Latinas in Eastern North Carolina. Violence Against Women, 13, 123-140.

Edleson, J. L. \& Tolman, R. M. (1992). Intervention for men who batter: An ecological approach. Newbury Park, CA: Sage.

Erez, E., \& Hartley, C. C. (2003). Battered immigrant women and the legal system: A therapeutic jurisprudence perspective. Western Criminology Review, 4, 155169.

Fagan, J., \& Browne, A. (1994). Violence between spouses and intimates: Physical aggression between men and women in intimate relationships. In: Reiss, A. J., Jr., and Roth, J. A. (eds.), Understanding and Preventing Violence, National Academy Press, Washington, DC, pp. 115-292.

Falicov, C. J. (1998). Latino families in therapy: A guide to multicultural practice. New York: Guilford Press.

Field, C. A., \& Caetano, R. (2004). Ethnic differences in intimate partner violence in the U.S. general population: The role of alcohol use and socioeconomic status. Trauma, Violence, \& Abuse, 5, 303-317. 
Work-related IPV 146

Firestone, J. M., Lambert, L. C., \& Vega, W. A. (1999). Intimate violence among Mexican origin women: Correlates of abuse. Journal of Gender, Culture, and Health, 4, 119-134.

Fitzgerald S., Dienenemann, J., \& Cadorette, M. F. (1998). Domestic violence in the workplace. American Association of Occupational Health Nurses Journal, 46, $345-353$.

Fontes, L. A. (2002). Child discipline and physical abuse in immigrant Latino families: Reducing violence and misunderstandings. Journal of Counseling and Development, 80, 31-40.

Galvez, G., Mankowski, E. S., Braun, M. F., \& Glass, N. (2009). Development of an iPod audio computer-assisted self-interview to increase the representation of low literacy populations in survey research. Field Methods, 21, 407-415.

Galvez, G., Mankowski, E. S., McGlade, M. S., Ruiz, M. E., \& Glass, N. (2011). Work-related intimate partner violence among employed immigrants from Mexico. Psychology of Men \& Masculinity.

Garcia, L., Hurwitz, E. L., \& Kraus, J. F. (2005). Acculturation and reported intimate partner violence among Latinas in Los Angeles. Journal of Interpersonal Violence, 20, 5, 569-590.

Gelles, R. J. (1985). Family violence. Annual Reviews, 11, 347-367.

Gerstman, B. B. (2003). Epidemiology kept simple: An introduction to traditional and modern epidemiology (2nd ed.). Hoboken, NJ: Wiley-Liss, 121-141. 
Griffith, J. D., Joe, G. W., Chatham, L. R., \& Simpson, D. D. (1998). The development and validation of a simpatia scale for Hispanics entering drug treatment. Hispanic Journal of Behavioral Sciences, 20, 468-482.

Grzywacz, J. G., Rao, P., Gentry, A., Marin, A., \& Arcury, T. A. (2009). Acculturation and conflict in Mexican immigrants' intimate partnerships: The role of women's labor force participation. Violence Against Women, 15, 11941212.

Guamaccia, P. J, Pincay, I. M., Alegria, M., Shrout, P., Lewis-Fernandez, R., Canino, G. (2007). Assessing diversity among Latinos: Results from the NLAAS. Hispanic Journal of Behavioral Sciences. 29, 510-534.

Harris, R. J., Firestone, J. M., \& Vega, W. A. (2005). The interaction of country of origin, acculturation, and gender role ideology on wife abuse. Social Science Quarterly, 86, 463-483.

Hazen, A. L. \& Soriano, F. I. (2007). Experiences with intimate partner violence among Latina women. Violence Against Women, 13, 562-582.

Healey, K., Smith, C., \& O’Sullivan, C. (1998). Batterer intervention: Program approaches and criminal justice strategies. Washington, D.C.: National Institute of Justice.

Herskovitz, M. J. (1938). Acculturation: The study of culture contact. New York: Augustin. 
Ho, C. K. (1990). An analysis of domestic violence in Asian American communities: A multicultural approach to counseling. Women \& Therapy, 9, 129-150.

Hovey, J.D. (2000). Psychosocial predictors of acculturative stress in Mexican immigrants. Journal of Psychology, 134, 490-502.

Hovey, J. D., \& King, C. A. (1996). Acculturative stress, depression, and suicidal ideation among immigrant and second-generation Latino adolescents. Journal of the American Academy of Child \& Adolescent Psychiatry. 35, 1183-1192.

Howell, D. (2009). New Material. In Statistical Home Page. Retrieved April 8, 2009, from http://www.uvm.edu/ dhowell/StatPages/StatHomePage.html.

Huo, Y. J., Smith, H. J., Tyler, T. R., \& Lind, E. A. (1996). Superordinate identification, subgroup identification and justice concerns: Is separatism the problem; is assimilation the answer? Psychological Science, 7, 40-45.

Hunt, L., Schneider, S., \& Comer, B. (2004). Should "acculturation" be a variable in health research? A critical review of research on U.S. Hispanics. Social Science and Medicine, 59, 973-986.

Ingram, E. M. (2007). A comparison of help seeking between Latino and non-Latino victims of intimate partner violence. Violence Against Women, 13, 159-171.

Jasinski, J. L. (1998). The role of acculturation in wife assault . Hispanic Journal of Behavioral Sciences, 20, 2, 175-191.

Kanuha, V. (1996). Domestic violence, racism and the battered women's movement in the United States. In J. L. Edelson \& Z. C. Eisikovits (Eds.), Future 
interventions with battered women and their families, 34-50. Thousand Oaks, CA: Sage Publications.

Kantor, G. K. (1997). Alcohol and spouse abuse: Ethnic differences. Recent Developments in Alcoholism, 13, 57-79.

Kantor, G., \& Jasinski, J. (1998). Dynamics and risk factors in partner violence. In J. Jasinski \& L. M. Williams (Eds.), Partner violence: A comprehensive review of 20 years of research (pp. 1-43). Thousand Oaks, CA: Sage.

Kantor, G. K., Jasinski, J. L., \& Aldorondo, E. (1994). Sociocultural status and incidents of marital violence in Latino families. Violence and Victims, 9, 207222.

Kasturirangan, A., Krishnan, S., \& Riger, S. (2004). The impact of culture and minority status on women's experience of domestic violence. Trauma, Violence, \& Abuse, 5, 318-332.

Kasturirangan, A \& Williams, E. (2003). Counseling Latina battered Women: A qualitiative study of the Latina perspective. Journal of Multicultural Counseling \& Development, 31, 162-178.

Kimmel, M. S. (2002). Gender symmetry in domestic violence: A substantive and methodological research review. Violence Against Women, 8, 1332-1363.

Klevens, J. (2007). An overview of intimate partner violence among Latinos. Violence Against Women, 13, 2, 111-122. 
Kroeber, A. L., \& Kluckholn, C. (1952). Culture: A critical review of concepts and definitions. Cambridge, MA: Peabody Museum, 47, 1.

Lim, K. C., Rioux, J. \& Ridley, E. (2004). Impact of domestic violence offenders on occupational safety \& health: A pilot study. Maine Department of Labor/Family Crisis Services.

Linton, R. (1936). The study of man. New York: Appleton-Century-Crofts.

Lipsky, S., Caetano, R., Field, C. A., \& Larkin, G. L. (2006). The role of intimate partner violence, race, ethnicity, in help-seeking behaviors. Ethnicity and Health, 11, 81-100.

Lloyd, S., \& Taluc, N. (1999). The effects of male violence on female employment. Violence Against Women, 5, 370-92.

Logan, TK., Shannon, L., Cole, J., \& Swanberg, J. (2007). Partner stalking and implications for women's employment. Journal of Interpersonal Violence, 22, 268.

Lown, E. A, Vega, W. A. (2001). Prevalence and predictors of physical partner abuse among Mexican American women. American Journal of Public Health, 91, $441-445$.

Malley-Morrison, K., \& Hines, D. A. (2004). Family violence in a cultural perspective: Defining, understanding, and combating abuse. Thousand Oaks, Ca: Sage Publications. 
Work-related IPV 151

MacMillan, R., \& Gartner, R. (1999). When she brings home the bacon: Labor-force participation and the risk of spousal violence against women. Journal of Marriage \& Family. 61: 947-958.

Mankowski, E. S., Galvez, G., Perrin, N., Hanson, G., Rollins, C. \& Glass, N. (2011). Assessment of Workplace Intimate Partner Violence: Perpetrator Behaviors and Impact, in preparation.

Marín, G. (1992). Issues in the measurement of acculturation among Hispanics. In K. F. Geisinger (Ed.), Psychological testing of Hispanics, APA Science Volumes (pp. 235-251). Washington, DC: American Psychological Association.

Marín, G., \& Gamba, R. J. (2003). Acculturation and changes in cultural values. In K. M. Chun, P. B. Organista, \& G. Marín (Eds.). Acculturation: Advances in theory, measurement and applied research. Washington, DC: American Psychological Association.

Marín, G. Marín VanOss, B. (1991). Research with Hispanic populations. Newbury Park, CA: Sage.

Marín, G., Sabogal, F., Marín, F. V., Otero-Sabogal, R., \& Perez-Stable, E. (1987). Development of a short acculturation scale for Hispanics. Hispanic Journal of Behavioral Sciences, 9, 183-205.

Mayo, Y. (1997). Machismo, fatherhood, and the Latino family: Understanding the concept. Journal of Multicultural Social Work, 5, 49-61. 
McGlade, M. S. (2002). Mexican farm labor networks and population increase in the Pacific Northwest. Association of Pacific Coast Geographers Yearbook, 64, $28-54$.

Mighty, E. J. (1997). Conceptualizing family violence as a workplace issue: A framework for research and practice. Employee Responsibilities and Rights Journal, 10, 249-262.

Mirandé, A. (1997). Hombres y machos: Masculinity and Latino culture. Westview Press, Boulder, CO.

Morash, M., Bui, H. N., \& Santiago, A. M. (2000). Cultural-specific gender ideology and wife abuse in Mexican-descent families. International Journal of Victimology, 7, 67-91.

Morbidity and Mortality Weekly Report (MMWR) (2008). Behavioral Risk Factor Surveillance System 2005: Summary Data Quality Report. Centers for Disease Control and Prevention. p. 113-117.

Moreno, C. L. (2007). The relationship between culture, gender, structural factors, abuse, trauma, and HIV/AIDS for Latinas. Qualitative Health Research, 17, 340.

National Center for Injury Prevention and Control (2003). Costs of intimate partner violence against women in the United States. Atlanta (GA): Centers for Disease Control and Prevention. 
Neff, J. A., Holman, B., \& Schluter, T. D. (1995). Spousal violence among Anglos, Blacks, and Mexican-Americans: The role of demographic variables, psychosocial predictors, and alcohol consumption. Journal of Family Violence $10,1-21$.

O’ Brien, K. (1993). Using focus groups to develop health surveys: An example from research on social relationships and AIDS-Preventive behavior. Health Education \& Behavior, 8, 361-372.

Orloff, L., E. \& Little, R. (1999). Somewhere to turn: Making domestic violence services accessible to battered immigrant women. A 'how to' manual for battered women's advocates and service providers. Washington, DC: AYUDA, Inc.

Oregon Human Development Corporation. (2003). Latina domestic violence best practices project: Final report.

Passel, J. S., Capps, R., \& Fix, M. (2004). Undocumented immigrants: Facts and figures. Washington, DC: The Urban Institute.

Passel, J. S. \& Cohn, D. (2008). U.S. population projections: 2005-2050. Washington, DC: The Pew Hispanic Center.

Pearlman, D. N., Zierler, S., Gjelsvik, A., Verhoek-Oftedahl, W. (2003). Neighborhood environment, racial position, and risk of police reported domestic violence: A contextual analysis. Public Health Reports, 118, 44-58. 
Perilla J. L., Bakeman, R., \& Norris, F. H. (1994). Culture and domestic violence: The ecology of abused Latinas. Violence and Victims, 9, 325-339.

Pew Hispanic Center. (2008). Demographic profile of Hispanics in Oregon, 2008.

Retrieved April 25, 2011 from http://pewhispanic.org/states/pdf/OR_08.pdf

Ramirez, R. R., \& de la Cruz, G. P. (2002). The Hispanic population in the United States: March 2002. U.S.Census Bureau, Washington DC.

Ridley, E., Rioux, J., Lim, K. C., Mason, D., Houghton, K. F., Luppi, F., \& Melody, T. (2005). Domestic violence survivors at work: How perpetrators impact employment. Maine Department of Labor/Family Crisis Services.

Riger, S., Ahrens, C., Blickenstaff, A. (2000). Measuring interference with employment and education reported by women with abusive partners: Preliminary data. Violence \& Victims, 15, 161-172.

Rodriguez, N., Consuelo, M. B., Paez, N. D., \& Myers, H. F. (2007). Exploring the complexities of familism and acculturation: Central constructs for people of Mexican origin. American Journal of Community Psychology, 39, 61-77.

Rothman, E. F. \& Corso, P. S. (2008). Propensity for intimate partner abuse and workplace productivity: Why employers should care. Violence Against Women, 14, 1054-1064.

Rothman, E., Stidsen, A., Hathaway, J. \& de Vries, H. (2007). How employment helps female victims of intimate partner violence: A qualitative study. Journal of Occupational Health Psychology, 12, 136-143. 
Rothman, E. F., \& Perry, M. J. (2004). Intimate partner abuse perpetrated by employees. Journal of Occupational Health Psychology, 9, 238-246.

Sabogal, F., Marín, G., Otero-Sabogal, R., Marín, B. V. \& Perez-Stable, E. J. (1987). Hispanic familism and acculturation: What changes and what doesn't? Hispanic Journal of Behavioral Sciences, 9, 397-412.

Saltzman, L. E., Fanslow, J. L., McMahon, P. M., \& Shelley, G. A. (1999). Intimate partner violence surveillance: Uniform definitions and recommended data elements, Version 1.0. Atlanta (GA): National Center for Injury Prevention and Control, Centers for Disease Control and Prevention.

Shepard, M. \& Pence, E. (1988). The effects of battering on employment status of women. Affilia, 3, 55-61.

Shetty, S. \& Kaguyutan, J. (2002). Immigrant victims of domestic violence: Cultural challenges and available legal protections. Harrisburg, PA: VAWnet, National Resource Center on Domestic Violence/Pennsylvania Coalition Against Domestic Violence. Retrieved March, 18, 2011 from: http://www.vawnet.org Sorenson, S. B., \& Telles, C. A. (1991). Self-reports of spousal violence in a Mexican American and non-Hispanic White population. Violence and Victims 6, 3-16.

Sorenson, S. B., Upchurch, D. M., \& Shen, H. (1996). Violence and injury in marital arguments: Risk patterns and gender differences. American Journal of Public Health, 86, 35-40. 
Stephenson, W. (1953). The study of behaviour: Q technique and its methodology. University of Chicago Press, Chicago.

Stevens, E. P. (1973) Marianismo: The other face of machismo in Latin America. In A. Pescatello (Ed.), Female and male in Latin America. University of Pittsburgh Press, PA.

Straus, M. A., Gelles, R. J., \& Steinmetz, S. K. (1980). Behind closed doors: Violence in the American family. Garden City, NY: Anchor Books.

Straus, M. A., Hamby, S. L., Boney-McCoy, S., \& Sugarman, D. B. (1996). The revised conflict tactics scales (CTS2): Development and preliminary psychometric data. Journal of Family Issues, 17, 283-316.

Straus, M. A., \& Smith, C. (1995). Violence in Latino families in the United States: Incidence rates and structural interpretations. In M. A. Straus \& R. J. Gelles (Eds.), Physical violence in American families: Risk factors and adaptations to violence in 8,145 families (pp. 341-367). New Brunswick, NJ: Transaction.

Sugarman, D. B., \& Frankel, S. L. (1996). Patriarchal ideology and wife-assault: A meta-analytic review. Journal of Family Violence, 11, 13-40.

Sugihara, Y., \& Warner, J. A. (2002). Dominance and domestic abuse among Mexican Americans: Gender differences in the etiology of violence in intimate relationships. Journal of Family Violence, 17, 315-340. 
Swanberg, J. E., Logan, T. K., \& Macke, C. (2005). Intimate partner violence, employment, and the workplace: Consequences and future directions. Trauma Violence Abuse, 6, 286-312.

Swanberg, J. E., \& Macke, C. (2006). Intimate partner violence and the workplace: Consequences and disclosure. Affilia, 21, 391-406.

Taylor, M. J., \& Barusch, A.S. (2004). Personal, family, and multiple barriers of longterm welfare recipients. Social Work, 49, 175-183.

Tafoya, S. (2004). Shades of belonging. Washington DC: Pew Hispanic Center.

Tjaden, P., \& Thoennes, N. (2000). Prevalence and consequences of male-to-female and female-to-male intimate partner violence as measured by the National Violence Against Women Survey. Violence Against Women, 6, 142-161.

Tolman, R. M., \& Wang, H. (2005). Domestic violence and women's employment: Fixed effects models of three waves of women's employment study data. American Journal of Community Psychology, 36, 147-158.

Toomela, A. (2003). How should culture be studied? Culture \& Psychology, 9, 35-45.

Torres, S. (1991). A comparison of wife abuse between two cultures: Perceptions, attitudes, nature, and extent. Issues in Mental Health Nursing, 12, 113-131.

Torres, J. B. (1998). Masculinity and gender roles among Puerto Rican men: A dilemma for Puerto Rican men's personal identity. American Journal of Orthopsychiatry, 68, 16-26. 
Torres, J. B., Solberg, V., Scott, H., \& Carlstrom, A. H. (2002). The myth of sameness among Latino men and their machismo. American Journal of Orthopsychiatry, $72,163$.

Triandis, H. C., Marín, G., Lisansky, J., \& Betancourt, H. (1984). Simpatia as a cultural script of Hispanics. Journal of Personality and Social Psychology, 47, 1363-1375.

Tylor, E. B. (1871). Primitive Culture. London: Murray.

U.S. Department of Labor, Bureau of Labor Statistics. (2007). Current population survey. Annual Averages 2006. U.S. Census Bureau, Washington DC.

U.S. Census Bureau. (2000). The Hispanic population in the United States. U.S. Census Bureau, Washington DC.

U.S. Census Data (2000). Profiles of general demographic characteristics: Oregon. U.S. Census Bureau, Washington DC.

Valdés, L. F., Barón, A., \& Ponce, F. Q. (1987). Counseling Hispanic men. In M. Scher, M. Stevens, G. Good, \& G. A. Eichenfield (Eds.), Handbook of counseling \& psychotherapy with men. Thousand Oaks, CA, US: Sage Publications, Inc.

Vasquez, M. J. (1998). Latinos and violence: mental health implications and strategies for clinicians. Cultural Diversity and Mental Health. 4, 319-334. 
West, C. M., Kantor, G. K., \& Jasinski, J. L. (1998). Sociodemographic predictors and cultural barriers to help-seeking behavior by Latina and non-Latino White American battered women. Violence and Victims, 13, 361-375.

Widiss, D. A. (2008). Domestic violence and the workplace: The explosion of state legislation and the need for a comprehensive strategy. Florida Statue University Law Review, 35, 669-728.

Williams, C. L. \& Berry, J. W. (1991). Primary prevention of acculturative stress among refugees: Application of psychological theory and practice. American Psychologist, 46, 632-41.

Wolf, M. E., Ly, U., Hobart, M. A., \& Kernie, M. A. (2003). Barriers to seeking police help for intimate partner violence. Journal of Family Violence, 18, 121-129.

Zambrano, M. (1985). Mejor sola que mal acompanada: For the Latina in an abusive relationship. Seattle: Seal Press. 